Copyright: The author, Kayleigh Ward, 2020.

\title{
Tropical Ideals and Discriminants
}

\author{
Kayleigh Ward
}

Submitted to Swansea University in fulfilment of the requirements for the Degree of

Doctor of Philosophy

Department of Mathematics

Swansea University

January 2020 


\title{
Tropical Ideals and Discriminants
}

\author{
Kayleigh Ward
}

\begin{abstract}
Tropical ideals arose in the work of Maclagan-Rincon and GiansiracusaGiansiracusa in the context of their scheme-theoretic refinement of tropicalization. An open problem is to understand what geometric information about a variety $V(I)$ is encoded in the tropical ideal trop $(I)$. In this thesis we focus on the valuation of the discriminant for certain classes of projective hypersurfaces of low-degree in both characteristic 0 and p. We find both cases where the valuation of the discriminant is determined by $\operatorname{trop}(\mathrm{I})$, and cases where it is not.
\end{abstract}




\section{Declarations and Statements}

\section{Declaration}

This work has not previously been accepted in substance for any degree and is not being concurrently submitted in candidature for any degree.

Signed (candidate):

\section{Statement 1}

This thesis is the result of my own investigations, except where otherwise stated. Other sources are acknowledged by giving explicit references. A bibliography is appended.

Signed (candidate):

\section{Statement 2}

I hereby give consent for my thesis, if accepted, to be available for photocopying and for inter-library loan, and for the title and summary to be made available to outside organisations.

Signed (candidate): 


\title{
TROPICAL IDEALS AND DISCRIMINANTS
}

\author{
KAYLEIGH WARD
}

\section{Contents}

1. Aknowledgements 4

2. Introduction 4

3. Background 6

3.1. A Brief Background in Classical Algebraic Geometry 6

3.2. Discriminants 9

$\begin{array}{lll}3.3 . & \text { Elliptic Curves and the } j \text {-invariant } & 10\end{array}$

\begin{tabular}{lll}
\hline 3.4. & Tropical Basics & 14
\end{tabular}

$\begin{array}{lll}3.5 . & \text { Tropical Initial Forms } & 17\end{array}$

3.6. Polyhedral Geometry and Newton Polygons 19

3.7. The Gröbner Complex 21

\begin{tabular}{cc|}
\hline $3.8 . \quad$ Kapranov's Theorem and the Fundamental Theorem of \\
Tropical Algebraic Geometry
\end{tabular}

$\begin{array}{lll}33.9 . & \text { Valuated Matroids } & 27\end{array}$

3.10. The Plücker Embedding and Tropical Linear Spaces 30

$\begin{array}{lll}3.11 . & \text { Tropical Ideals } & 34\end{array}$

3.12. Tropical Elliptic Curves and the $j$-invariant 35

\begin{tabular}{lll}
\hline $4 . \quad$ Quadratics and Generalisations & 38
\end{tabular}

\begin{tabular}{lll}
\hline 4.1. & The Quadratic Case & 39
\end{tabular}

4.2. Patterns in Plücker Coordinates 44

4.3. The Quadratic Case in Projective 2-space: Ideals of the

\begin{tabular}{|r|r}
\hline Form $\left\langle y^{2}+x^{2}+B x z+C z^{2}\right\rangle$ & 46 \\
\hline
\end{tabular}

4.4. Biquadratics and Beyond 50

4.5. The Multiquadratic Case in Projective 2-space: Ideals of the Form $\left\langle y^{2 n}+x^{2 n}+B x^{n} z^{n}+C z^{2 n}\right\rangle \mid 56$

\begin{tabular}{ll|l} 
5. $\quad$ Generalising to Degree 3 & 61
\end{tabular}

5.1. Cubics of the Form $x^{3}+A x z^{2}+B z^{3} \quad 61$

5.2. Two Tropical Cubics 66

5.3. $\quad$ Proof That $v\left(\Delta_{3}\right)$ Cannot be Recovered from trop $\left(\left\langle x^{3}+\right.\right.$ $\left.\left.A x z^{2}+B z^{3}\right\rangle\right)$. 68

5.4. Polynomials of the form $x^{n}+A x z^{n-1}+B z^{n} \quad 70$

5.5. The Plücker Coodinates of $\left\langle x^{3}+A x z^{2}+B z^{3}\right\rangle$ and $\Delta$ in Fields of Characteristic $p$ - 71

5.6. The Plücker Coordinates of $\left\langle y^{2} z-x^{3}-A x z^{2}-B z^{3}\right\rangle \quad 74$

Date: December 2019. 


\begin{tabular}{|lr|}
\hline 5.7. The Plücker Coordinates of Tropical Elliptic Curves in \\
\hline \multicolumn{2}{|c|}{ Characteristic $p$} \\
\hline 5.8. Cubics of the Form $x^{3}+B x^{2} z+D z^{3}$ \\
\hline 5.9. The General Cubic $A x^{3}+B x^{2} z+C x z^{2}+D z^{3}$ & 76 \\
\hline $6 . \quad$ Open Questions and Conclusion & 77 \\
\hline \hline References & 80 \\
\hline
\end{tabular}

\section{Aknowledgements}

I would like to thank my supervisor Prof. Jeffrey Giansiracusa for all of his support, patience, and hard work over the past few years, and for being a great person to sit down and work through mathematics with whenever I found myself stuck.

I would also like to give a general thanks to all the mathematicians currently working in tropical geometry and related fields, whose research and talks have inspired and will continue to inspire me to want to learn more.

I could not have completed this work without the support of my parents, who have always done their best to help me to achieve my goals, and my son, who makes my achievements feel worthwhile. I would also like to thank all those closest to me, who have given me all the love and support I could ever ask for.

\section{INTRODUCTION}

Tropical geometry is a relatively new and active area of research, and there are tropical analogues of many important concepts in classical algebraic geometry. Much of the research into tropical geometry focuses on finding appropriate tropical analogues to classical algebraic structures and invariants. In their paper [8], Jeffrey and Noah Giansiracusa introduce the concept of tropical schemes which give us additional data, in a manner analogous to the way that classical schemes encode more information than varieties. In their paper [16], Maclagan and Rincon define tropical ideals, a special subset of ideals of $\mathbb{T}\left[x_{1}, \ldots, x_{n}\right]$ which have particularly nice properties, including satisfying an ascending chain condition and a version of the Nullstellensatz.

The work in this thesis is inspired by a question arising due to the work of Katz, Markwig and Markwig in their papers [12] and [13] on tropical elliptic curves and their $j$-invariants. It turns out that when the tropicalisation of an elliptic curve $\mathcal{E}$ has a cycle, the appropriate tropical analogue to the $j$-invariant is the negative of the length of this cycle. They also define a generalised cycle length so that in certain cases we can find the tropical $j$-invariant of curves with no cycle. However, there are some cases where this does not work. We therefore ask whether the valuation of the $j$-invariant is determined by the valuations of the 
Plücker coordinates of the linear space of the ideal generated by the defining polynomial of $\mathcal{E}$.

The question above involves studying hypersurfaces of $\mathbb{P}^{2}$ (1-dimensional subschemes) over the complex Puiseux series $\mathbb{C}\{\{t\}\}$, and turns out to be more difficult than we anticipated. Initially we turn our attention to hypersurfaces of $\mathbb{P}^{1}$ over $\mathbb{C}\{\{t\}\}$ instead. In their paper [9], Fink, Giansiracusa and Giansiracusa tackle the question of whether the valuation of the discriminant of a quadratic $A x^{2}+B x z+C z^{2}$ is determined by the valuated Plücker coordinates of its corresponding tropical ideal, and find that it is not. We follow on from this work in Section 4.4 by generalising the result to ideals generated by multiquadratics of the form $x^{2 n}+B x^{n} z^{n}+C z^{n}$, as well as investigating in 4.1.4 and 4.4.1 whether the result is true over fields of characteristic $p$ for certain small values of $p$. We find that the valuations of the Plücker coordinates do determine the valuation of the discriminant for $p=3,5,7,11$.

In Section 5.1 we move closer to addressing the question of $j$-invariants by investigating the relationship between the valuation of the discriminant of a cubic $x^{3}+A x z^{2}+B z^{3}$ and the valuated Plücker coordinates of the ideal it generates. Similarly to the quadratic case, we find that the valuation of the discriminant does not depend on the valuations of the Plücker coordinates, and we generalise this result to trinomials of the form $x^{n}+A x z^{n-1}+B z^{n}$ in Section 5.4. In Section 5.5 we also show that over fields of characteristic $p$ the valuation of the discriminant does depend on the valuated Plücker coordinates.

In the case of quadratics, multiquadratics, and cubics in Weierstrass form, all of the Plücker coordinates can be described by a single recurrence relation, but the same cannot be said for the general cubic. In fact, we see in Section 5.8 that the Plücker coordinates of cubics of the form $x^{3}+B x^{2} z+C z^{3}$ cannot be described by a single recurrence relation. We give a partial result for such cubics, that the valuation of the discriminant is not determined by the valuated Plücker coordinates described by a recurrence relation that arises from a single family of minors, however it is still unclear whether there is a convenient way to describe all of the Plücker coordinates, and whether the valuation of the discriminant depends on their valuations. The general cubic case is even more complex. Since elliptic curves over fields of characteristic 2 and 3 cannot necessarily be expressed in Weierstrass form via a change of variables, it would be useful to better understand the Plücker coordinates of the ideals generated by more general cubics.

Finally, moving onto hypersurfaces of $\mathbb{P}^{2}$, we begin in Section 4.3 by looking at the Plücker coordinates of ideals generated by quadrics of the form $y^{2}+x^{2}+B x z+C z^{2}$. Although we do not characterise all Plücker coordinates of such ideals, we can identify using Macaulay2 that in degree 5 the discriminant of the polynomial $x^{2}+B x z+C z^{2}$ appears as a factor in many of the Plücker coordinates. Thus if the 
valuation of this discriminant deviates from the generic valuation, then there will be Plücker coordinates whose valuations also deviate from their generic valuations. Thus if any one of these does not deviate from its generic valuation, we know that the discriminant does not deviate from its generic valuation. We generalise this to ideals of the form $\left\langle y^{4}+x^{4}+B x^{2} z^{2}+C z^{4}\right\rangle$, and conjecture that it can in fact be generalised to all ideals of the form $\left\langle y^{2 n}+x^{2 n}+B x^{n} z^{n}+C z^{2 n}\right\rangle$. We consider elliptic curves over fields of characteristic 0 in Section 5.6, and over characteristic $p$ in Section 5.7. Since describing the Plücker coordinates of the ideal defined by a polynomial of the form $y^{2} z-x^{3}-A x z^{2}-B z^{3}$ turned out to be harder than we expected, in the former case we cannot give a full answer, though in the latter case we prove that the valuation of the $j$-invariant does in fact depend on the valuated Plücker coordinates.

\section{BACKGROUND}

The main reference for this section, unless otherwise noted, is Diane Maclagan and Bernd Sturmfels' book Introduction to Tropical Geometry [17. We will cover the basics of tropical algebra and tropical geometry, including tropical varieties, tropical schemes, and their relationship to valuated matroids. Our aim will be to build a picture of what we set out to study and learn, as well as lay the foundations for understanding the analysis and results in the sections that follow.

3.1. A Brief Background in Classical Algebraic Geometry. In this section we will briefly recall some ideas from classical algebraic geometry, the tropical counterparts of which we will meet later. An active area of current research in tropical geometry is not only in finding tropical counterparts to more familiar algebraic structures and invariants, but in working out what information is retained when we pass to the tropical world.

Definition 3.1.1. Let $k$ be a field, and let $k\left[x_{1}, \ldots, x_{n}\right]$ be the ring of polynomials in $n$ variables with coefficients in $k$. Let $I \subset k\left[x_{1}, \ldots, x_{n}\right]$ be an ideal. We define the following:

(1) Let $\mathbf{V}(I)$ be the set $\left\{\mathbf{a} \in k^{n}: f(\mathbf{a})=0 \forall f \in I\right\}$. This is the vanishing locus of $I$, which we call the affine variety of $I$.

(2) Let $J$ be a subset of $k^{n}$. Then we define $\mathcal{I}(J)$ to be the set $\left\{f \in k\left[x_{1}, \ldots, x_{n}\right]: f(\mathbf{a})=0 \forall \mathbf{a} \in J\right\}$, i.e., the set of all polynomials which vanish on every point in $J$.

(3) For an affine variety $\mathbf{V}$, we define its coordinate ring to be the quotient $k\left[x_{1}, \ldots, x_{n}\right] / \mathcal{I}(\mathbf{V})$, which we will denote by $k[\mathbf{V}]$.

Definition 3.1.2. We refer to an $(n-1)$-dimensional variety embedded in affine $n$-space as a hypersurface. 
The next result (a proof for which can be found in any introductory text on algebraic geometry) tells us that there is a one-to-one, orderreversing correspondence between (irreducible) affine algebraic varieties and radical ideals (that is, ideals $J \in k\left[x_{1}, \ldots, x_{n}\right]$ for which $J=\sqrt{J}=$ $\left.\left\{f \in k\left[x_{1}, \ldots, x_{n}\right]: f^{n} \in J, n \in \mathbb{N}\right\}\right)$. The idea of this correspondence will come up later when we look at schemes.

Theorem 3.1.3 (Hilbert's Nullstellensatz). Let $k$ be an algebraically closed field, and let $I \subset k\left[x_{1}, \ldots, x_{n}\right]$ be an ideal. Then $\mathcal{I}(\boldsymbol{V}(I))=\sqrt{I}$.

Example 3.1.4. Consider the polynomial

$$
p(x)=(x+a)(x-a)=x^{2}-a^{2}
$$

over $\mathbb{C}[x]$, for some $a \in \mathbb{C}$. Let $I=\left\langle x^{2}-a^{2}\right\rangle=\langle x+a\rangle \cap\langle x-a\rangle$. Then $\mathbf{V}(I)=\{a,-a\}, \mathcal{I}(\mathbf{V}(I))=\left\langle x^{2}-a^{2}\right\rangle$ (since $I$ is a radical ideal), and $\mathbb{C}[\mathbf{V}(I)]=\mathbb{C}[x] /\left\langle x^{2}-a^{2}\right\rangle$.

Now consider the polynomial

$$
q(x)=(x+a)(x-a)(x+a i)(x-a i)=x^{4}-a^{4}
$$

over $\mathbb{C}[x]$, for some $a \in \mathbb{C}$. Let $J=\left\langle x^{4}-a^{4}\right\rangle=\bigcap_{0 \leq j \leq 3}\left\langle x+i^{j} a\right\rangle$, so that $\mathbf{V}(J)=\{a,-a, a i,-a i\}$. Then $\mathcal{I}(\mathbf{V}(J))=\left\langle x^{4}-a^{4}\right\rangle$ (since $J$ is a radical ideal), and $\mathbb{C}[\mathbf{V}(J)]=\mathbb{C}[x] /\left\langle x^{4}-a^{4}\right\rangle$.

Note that $J \subset I$ and $\mathbf{V}(I) \subset \mathbf{V}(J)$.

Definition 3.1.5. Let $\mathbb{P}_{k}^{n}$ be the set of $(n+1)$-tuples $\left[a_{0}: \ldots: a_{n}\right]$ modulo the relation $\left[a_{0}: \ldots: a_{n}\right] \sim\left[k a_{0}: \ldots: k a_{n}\right]$ for all $k \in k \backslash\{0\}$ (or equivalently, the set of all lines through the origin in $k^{n+1}$ ). We call this projective $n$-space over $k$. Projective varieties can be defined in much the same way as affine varieties. Let $I \subset k\left[x_{0}, \ldots, x_{n}\right]$ be a homogeneous ideal (i.e., an ideal generated by homogeneous polynomials, where the sum of the indices of each monomial is the same). We define:

(1) Let $\mathbf{V}(I)$ be the set $\left\{\mathbf{a} \in k^{n+1}: f(\mathbf{a})=0 \forall f \in I\right\}$. This is the vanishing locus of $I$, which we call the projective variety of $I$.

(2) Let $J$ be a subset of $k^{n+1}$. Then we define $\mathcal{I}(J)$ to be the set $\left\{f \in k\left[x_{0}, \ldots, x_{n}\right]: f(\mathbf{a})=0 \forall \mathbf{a} \in J\right\}$, i.e., the set of all polynomials which vanish on every point in $J$.

(3) For a projective variety $\mathbf{V}$, we define its homogeneous coordinate ring to be the quotient $k\left[x_{0}, \ldots, x_{n}\right] / \mathcal{I}(\mathbf{V})$, which we will denote by $k[\mathbf{V}]$.

There is a projective version of the Nullstellensatz so that we also have a one-to-one order-reversing correspondence between radical homogeneous ideals and projective varieties.

Definition 3.1.6. The dimension $d$ of an affine algebraic variety $V$ is defined to be the maximal length of the chains $V_{0} \subset V_{1} \subset \ldots \subset V_{d}$ of irreducible subvarieties (i.e., subsets of $V$ which are themselves varieties) 
of $V$ (this definition can equivalently be phrased in terms of chains of prime ideals in the coordinate ring of $V$ ).

A hypersurface in affine (or projective) $n$-space is an $(n-1)$-dimensional affine (or projective) algebraic variety.

Definition 3.1.7 (Zariski Topology). The Zariski topology on $\mathbb{P}^{n}$ is defined by specifying the closed sets to be the projective algebraic sets

$$
\mathbf{V}(S)=\left\{\mathbf{a} \in k^{n+1}: f(\mathbf{a})=0 \forall f \in S\right\},
$$

where $S$ is a set of homogeneous polynomials.

Now let $k[\mathbf{V}]$ be the homogeneous coordinate ring of a projective variety $\mathbf{V}, S$ be as above, and let $T$ be the image of $S$ in $k[\mathbf{V}]$ Then the subset of $\mathbf{V}$ given by

$$
\mathbf{V}^{\prime}(T)=\{\mathbf{a} \in \mathbf{V}: f(\mathbf{a})=0 \forall f \in T\}=\mathbf{V} \cap \mathbf{V}(S),
$$

and so $k[\mathbf{V}]$ inherits the Zariski topology from $\mathbb{P}^{n}$ via the subspace topology.

Definition 3.1.8. The $n$-dimensional torus over a field $\mathbb{K}$ is defined to be the affine open subset

$$
\left(\mathbb{K}^{\star}\right)^{n}=\mathbb{K}^{n} \backslash \mathbf{V}\left(x_{1}, \ldots, x_{n}\right) \subset \mathbb{K}^{n},
$$

with coordinate ring

$$
\mathbb{K}\left[x_{1}, \ldots, x_{n}\right]_{x_{1} \ldots x_{n}}=\mathbb{K}\left[x_{1}^{ \pm 1}, \ldots, x_{n}^{ \pm 1}\right],
$$

i.e., the field of fractions of $\mathbb{K}\left[x_{1}, \ldots, x_{n}\right]$. The torus $\left(\mathbb{K}^{\star}\right)^{n}$ is a group under componentwise multiplication. A torus $T$ is an affine variety isomorphic to $\left(\mathbb{K}^{\star}\right)^{n}$, which inherits the group structure from the isomorphism. We can (loosely) define an affine toric variety $V$ to be an irreducible affine variety containing a torus $\left(\mathbb{K}^{\star}\right)^{n}$ as a Zariski open subset.

A detailed account of toric varieties can be found in [3]. For the most part (for the purposes of this work) we will not need to concern ourselves with the intricacies of toric varieties, but the definition is included for completeness.

Definition 3.1.9. A subset $A$ of a topological space $X$ is called dense in $X$ if every point $x \in X$ either belongs to $A$ or is a limit point of $A$. We say that a subset $A$ of $X$ is Zariski dense if it is dense in $X$ with respect to the Zariski topology.

Scheme theory is an extension of the theory of varieties that encodes more information (for example, multiplicities of points - see Example 3.1 .10 below). Just like the correspondence between affine algebraic varieties and radical ideals of polynomial rings, we have a one-to-one order-reversing correspondence between subschemes of affine $n$-space and general ideals of $\mathbb{K}\left[x_{1}, \ldots, x_{n}\right]$. We will avoid going too much into the technical details of schemes here, and instead give an example that 
demonstrates some of the extra information encoded in the scheme structure.

The points of an affine scheme Spec $R$ are defined to be the prime ideals $\mathfrak{p}$ of the commutative ring $R$. At each point $\mathfrak{p}$ of $\operatorname{Spec} R$ we define the local ring $R_{\mathfrak{p}}$ to be the localisation at $\mathfrak{p}$, which has the unique maximal ideal $\mathfrak{p} R_{\mathfrak{p}}$. The residue field of the point $\mathfrak{p}$ is then defined to be the quotient $R_{\mathfrak{p}} / \mathfrak{p} R_{\mathfrak{p}}$. Define a regular function to be an element $r \in R$. We say that a regular function $r$ vanishes on $\mathfrak{p}$ if $r \in \mathfrak{p}$, and $r$ vanishes on $\operatorname{Spec} R$ if it vanishes at every point of $\operatorname{Spec} R$.

Example 3.1.10. Let's return to $p(x)$ and $q(x)$ from Example 3.1.4. and consider what happens at the limit as $a \rightarrow 0 . I=\left\langle x^{2}\right\rangle$ and $J=\left\langle x^{4}\right\rangle$ are no longer radical ideals. We get that $\mathbf{V}(I)=\mathbf{V}(J)=\{0\}$, and $\mathcal{I}(\mathbf{V}(I))=\mathcal{I}(\mathbf{V}(J))=\langle x\rangle$. Thus both $\mathbf{V}(I)$ and $\mathbf{V}(J)$ have coordinate ring $\mathbb{C}[x] /\langle x\rangle \cong \mathbb{C}$.

Intuitively, for nonzero $a$, the cardinalities of $\mathbf{V}(I)$ and $\mathbf{V}(J)$ are 2 and 4 respectively. As a approaches zero, these points get closer and closer together so that at the limit we get a double and quadruple point respectvely.

Both $X=\operatorname{Spec} \mathbb{C}[x] /\left\langle x^{2}\right\rangle$ and $Y=\operatorname{Spec} \mathbb{C}[x] /\left\langle x^{4}\right\rangle$ have the single point $\langle x\rangle$. In the case of $X$, we find that there exist non-zero nilpotent regular functions of the form $k x$ for some $k \in \mathbb{C}$ that vanish on the point $\langle x\rangle$; in the case of $Y$, there exist non-zero nilpotent regular functions of the form $a x^{3}+b x^{2}+c x$ for $a, b, c \in \mathbb{C}$ that vanish on the point $\langle x\rangle$.

3.2. Discriminants. The discriminant of a polynomial is a function of its roots (or its coefficients, which are also functions of its roots), and is a tool we can use to determine singular loci in the hypersurface it generates. We will predominantly work with the definition using the coefficients, but for completeness we will give the definition in terms of roots first.

Definition 3.2.1. The discriminant of a polynomial $p(x)=x^{n}+$ $a_{n-1} x^{n-1}+\ldots+a_{1} x+a_{0}$, with roots $p_{1}, \ldots, p_{n}$ is given by the formula:

$$
\prod_{i<j}\left(p_{i}-p_{j}\right)
$$

Notice that if any two of the roots are equal, it vanishes.

The second definition of the discriminant requires us first to define the resultant of two polynomials. This definition will be more useful to us later for proving general forms of certain discriminants, as it is easier to see how they arise from the Sylvester matrix.

Definition 3.2.2. The Sylvester matrix of two polynomials $p(x)$ of degree $m$ and $q(x)$ of degree $n$, with coefficients in a field $\mathbb{K}$, is the $(n+m) \times(m+n)$ square matrix defined as follows. If $p(x)=a_{m} x^{m}+$ 
$a_{m-1} x^{m-1}+\ldots+a_{0}$, the first $n$ rows are as shown:

$$
\left[\begin{array}{cccccccc}
a_{m} & a_{m-1} & a_{m-2} & \ldots & a_{0} & 0 & \ldots 0 & 0 \\
0 & a_{m} & a_{m-1} & \ldots & a_{1} & a_{0} & \ldots 0 & 0 \\
0 & 0 & a_{m} & \ldots & a_{2} & a_{1} & \ldots 0 & 0 \\
\vdots & & & & & \ddots & & \vdots \\
0 & 0 & & \ldots & & & a_{1} & a_{0}
\end{array}\right]
$$

The bottom $m$ rows consist of the coefficients of $q(x)$ arranged in a similar manner.

We define the resultant $\operatorname{Res}(p(x), q(x))$ of $p(x)$ and $q(x)$ to be the determinant of this square matrix. The resultant of $p(x)$ and $q(x)$ is a polynomial in their coefficients which is zero if and only if they share a common divisor of positive degree, i.e., they share at least one root in some algebraically closed field extension of $\mathbb{K}$.

The polynomial $p(x)=a_{m} x^{m}+a_{m-1} x^{m-1}+\ldots+a_{0}$ has a repeated root if and only if it shares at least one root with its derivative $p^{\prime}(x)=$ $m a_{m} x^{m-1}+(m-1) a_{m-1} x^{m-2}+\ldots+a_{1}$. Thus we can take the resultant $\operatorname{Res}\left(p(x), p^{\prime}(x)\right)$ to obtain a polynomial in the coefficients $a_{i}$ that is zero if and only if $p(x)$ has a repeated root. It can be shown that

$$
\operatorname{Res}\left(p(x), p^{\prime}(x)\right)=(-1)^{\frac{m(m-1)}{2}} a_{m} \Delta,
$$

where $\Delta$ is the discriminant as defined above in terms of the roots.

Following on from Section 3.1, the discriminant is a useful tool to quickly determine whether the scheme determined by $p(x)$ has singular loci (e.g. a double point).

3.3. Elliptic Curves and the $j$-invariant. In this section we will briefly give some background on elliptic curves, over fields of characteristic 0 and of prime characteristic $p$. The main reference used is Elliptic Curves - Number Theory and Cryptography by Lawrence C Washington [19].

3.3.1. In Characteristic 0. An elliptic curve over an algebraically closed field $\mathbb{K}$ can be defined as a nonsingular projective curve of genus 1 . We will mostly be concerned with elliptic curves in Weierstrass form, since over a field of characteristic 0 any elliptic curve can be defined by an equation of the form $y^{2}=x^{3}+A x+B$. Starting with the generalised Weierstrass equation $y^{2}+a_{1} x y+a_{3} y=x^{3}+a_{2} x^{2}+a_{4} x+a_{6}$, where the $a_{i}$ are constants, we can complete the square and use a change of variables to get the required equation in Weierstrass form. See Example 3.3 .4 below.

Definition 3.3.1. An elliptic curve over $\mathbb{K}$ is a curve given by the equation $y^{2} z=x^{3}+A x z^{2}+B z^{3}$ for $A, B \in \mathbb{K}$. For the purposes of this work, we will only consider elliptic curves in a projective space. 
The $j$-invariant of such an elliptic curve is given by the following formula:

$$
j=1728 \frac{4 A^{3}}{4 A^{3}+27 B^{2}} .
$$

Note that if $\mathbb{K}$ is not algebraically closed, it is possible to have two elliptic curves with the same $j$-invariant, for which an isomorphism can't be found without passing to some extension of $\mathbb{K}$.

The relationship between two ellitptic curves with the same $j$-invariant is stated explicitly in the result below, which we will not prove, but we will illustrate with Example 3.3.3.

Theorem 3.3.2 (Theorem 2.19 [19]). Let $y_{1}^{2}=x_{1}^{3}+A_{1} x_{1}+B_{1}$ and $y_{2}^{2}=x_{2}^{3}+A_{2} x_{2}+B_{2}$ be elliptic curves over a field $\mathbb{K}$ (not of characteristic 2 or 3 ) with $j$-invariants $j_{1}$ and $j_{2}$ respectively. If $j_{1}=j_{2}$, then there exists $0 \neq \mu \in \overline{\mathbb{K}}$ such that

$$
A_{2}=\mu^{4} A_{1}, \quad B_{2}=\mu^{6} B_{1} .
$$

The transformation taking one to the other is given by

$$
x_{2}=\mu^{2} x_{1}, \quad y_{2}=\mu^{3} y_{1} .
$$

This gives an isomorphism.

Example 3 3.3. Let $\mathcal{E}_{1}$ be an elliptic curve over $\mathbb{Q}$ defined by the equation

$$
y^{2}=x^{3}+4 x+3 .
$$

We find that $\mathcal{E}_{1}$ has $j$-invariant $\frac{442368}{449}$. Now let $\mathcal{E}_{2}$ be the elliptic curve over $\mathbb{Q}$ defined by the equation

$$
y_{1}^{2}=x_{1}^{3}+16 x_{1}-24 .
$$

We find that $\mathcal{E}_{2}$ has the same $j$-invariant. Using the above theorem we have $\mu=\sqrt{2} i$, which is not in $\mathbb{Q}$ itself but in the extension $\mathbb{Q}[\sqrt{2} i]$.

Example 3.3.4. Let $\mathcal{E}$ be the elliptic curve over $\mathbb{C}\{\{t\}\}$, the field of complex Puiseux series (see 3.4 .6 for a full definition), defined by the equation

$$
y^{2}+t x y+t^{2} y=x^{3}+\left(t+t^{2}\right) x^{2}+t^{-1} x+\left(1+t^{2}\right) .
$$

To put this into Weierstrass form, we first complete the square to get

$$
y^{2}+t x y+t^{2} y=\left(y+\frac{t x+t^{2}}{2}\right)^{2}-\left[\frac{t^{2} x^{2}}{4}+\frac{t^{3} x}{2}+\frac{t^{4}}{4}\right]
$$

then putting $y_{1}=y+\frac{t x+t^{2}}{2}$ yields

$$
y_{1}^{2}=x^{3}+\left(\frac{4 t+5 t^{2}}{4}\right) x^{2}+\left(\frac{2 t^{-1}+t^{3}}{2}\right) x+\left(\frac{4+4 t^{2}+t^{4}}{4}\right) .
$$


We now make the change of variables $x=x_{1}-\frac{4 t+5 t^{2}}{12}$ which results in the coefficients of $x_{1}^{2}$ cancelling out. This gives us the new equation

$$
\begin{aligned}
y_{1}^{2} & =x_{1}^{3}+\frac{1}{48}\left(48 t^{-1}-16 t^{2}-16 t^{3}-25 t^{4}\right) x_{1} \\
& +\frac{1}{864}\left(576-360 t+864 t^{2}+64 t^{3}+312 t^{4}+120 t^{5}+125 t^{6}\right),
\end{aligned}
$$

as required.

In general we can follow the same process for any elliptic curve defined by the equation

$$
y^{2}+a_{1} x y+a_{3} y=x^{3}+a_{2} x^{2}+a_{4} x+a_{6},
$$

and note that this is possible in a field of any characteristic except 2 and 3 , since we must divide by 2 and 3 .

3.3.2. In Characteristic $p$. For $p \geq 5$, the situation is the same as in characteristic 0 . However, for characteristic 2 and 3 , we need to take more care. The process required to convert an elliptic curve to Weierstrass form as shown in Example 3.3.4 involves division by 2 (when completing the square) and 3 (in the change of variables), which are not possible in characteristics 2 and 3 respectively.

Definition 3.3.5. In characteristic 3, any elliptic curve can, via a change of variables, be expressed in the form $y^{2}=x^{3}+B x^{2}+C x+D$, and its $j$-invariant is given by the formula

$$
j=\frac{B^{6}}{B^{2} C^{2}-C^{3}-B^{3} D} .
$$

In characteristic 2 , we have two possibilities. Either we can express the elliptic curve in the form $y^{2}+x y=x^{3}+C x+D$, and it has $j$-invariant $\frac{1}{D}$, or we can express the curve in the form $y^{2}+E y=x^{3}+C x+D$, and it has $j$-invariant 0 .

The $j$-invariant can be calculated directly from the generalised Weierstrass equation (see, for example, Appendix 1 of [14]), which is where these formulas come from, but we won't derive them here.

Example 3.3.6. Let $\mathcal{E}$ be the elliptic curve in $\mathbb{K}$, a field of characteristic 2 , defined by the equation

$$
y^{2}+x y=x^{3}+\lambda x+(\lambda+1),
$$

where $\lambda$ is such that $\lambda^{2}=\lambda+1$. By the above formula, its $j$-invariant is given by $\frac{1}{\lambda+1}=\lambda$.

Example 3.3.7. Let $\omega$ be such that $\omega^{2}=2$ in $\mathbb{K}$, a field of characteristic 3 . Let $\mathcal{E}$ be the elliptic curve defined by the equation

$$
y^{2}=x^{3}+(1+\omega) x^{2}+2 x+(2+\omega) .
$$


Its $j$-invariant is given by

$$
\begin{aligned}
j & =\frac{(1+\omega)^{6}}{(1+\omega)^{2} \cdot 2^{2}-(1+\omega)^{3}(2+\omega)-2^{3}} \\
& =\frac{\omega}{2 \omega-2 \omega-2} \\
& =\frac{\omega}{-2}=\omega .
\end{aligned}
$$

Here are a few more results about elliptic curves in characteristic 3 , taken from Exercise 2.18 of [19]. Let $\mathbb{K}$ be any field of characteristic 3 and let $y^{2}=x^{3}+B x^{2}+C x+D$ be an elliptic curve.

First, note that in characteristic 3 , we have the identity $(x+u)^{3}=$ $x^{3}+u^{3}$, which means that the curve $y^{2}=x^{3}+D$ always has a triple root, so we assume that at least one of $B$ and $C$ is nonzero. Now suppose that $B \neq 0$, and make the change of variables $x \mapsto x_{1}+\frac{C}{B}$ :

$$
\begin{aligned}
y^{2} & =\left(x_{1}+\frac{C}{B}\right)^{3}+B\left(x_{1}+\frac{C}{B}\right)^{2}+C\left(x_{1}+\frac{C}{B}\right)+D \\
& =x_{1}^{3}+\frac{C^{3}}{B^{3}}+B x_{1}^{2}+2 C x_{1}+\frac{C^{2}}{B^{2}}+C x_{1}+\frac{C^{2}}{B}+D \\
& =x_{1}^{3}+B x_{1}^{2}+\frac{C^{3}+C^{2} B+C^{2} B^{2}+D B^{3}}{B^{3}}
\end{aligned}
$$

So we can assume that exactly one of $B$ and $C$ is equal to zero.

Suppose that $\mathcal{E}_{1}$ and $\mathcal{E}_{2}$ are elliptic curves determined by the equations $y^{2}=x^{3}+B_{1} x^{2}+D_{1}$ and $y^{2}=x^{3}+B_{2} x^{2}+D_{2}$ respectively, and suppose that they have the same $j$-invariant. Note that since $C=0$, the formula for the $j$-invariant becomes

$$
j=\frac{-B^{3}}{D} .
$$

We choose some $\mu$ such that $\mu^{2} B_{1}=B_{2}$, and consider the $j$-invariant. Because $j\left(\mathcal{E}_{1}\right)=j\left(\mathcal{E}_{2}\right)$ we have

$$
\frac{-B_{2}^{3}}{D_{2}}=\frac{-\left(\mu^{2} B_{1}\right)^{3}}{D_{1}}=\frac{-\mu^{6} B_{1}^{3}}{D_{2}},
$$

which implies that $D_{2}=\mu^{6} D_{1}$. In summary, we have

Proposition 3.3.8. If two elliptic curves over a field $\mathbb{K}$ of characteristic $3, \mathcal{E}_{1}: y^{2}=x^{3}+B_{1} x^{2}+D_{1}$ and $\mathcal{E}_{2}: y^{2}=x^{3}+B_{2} x^{2}+D_{2}$ have the same $j$-invariant, then there exists some $\mu \in \overline{\mathbb{K}}$ such that $B_{2}=\mu^{2} B_{1}$ and $D_{2}=\mu^{6} D_{1}$.

Now, note that if $B=0$, we have $j=0$, and if $C=0$, we have $j=\frac{-B^{3}}{D}$. Suppose $B=2$ so that $-B^{3}=1$, then $j=\frac{1}{D}$. For any element $u \in \mathbb{K}$, the curve defined by the equation $y^{2}=x^{3}+2 x^{2}+u^{-1}$ has $j$-invariant $u$. Thus every element of $\mathbb{K}$ arises as the $j$-invariant of some elliptic curve over $\mathbb{K}$. 
Example 3.3.9. Take $\mathbb{K}=\mathbb{F}_{9}$ with $\omega^{2}=2$ as before, and take $u=$ $1+2 \omega$. Then $u^{-1}=2+2 \omega$ and the elliptic curve defined by the equation $y^{2}=x^{3}+2 x^{2}+(2+2 \omega)$ has $j$-invariant $1+2 \omega$.

3.4. Tropical Basics. This section provides the basic definitions we will need to begin working in the tropical world.

\subsubsection{Valuations and the Tropical Semiring.}

Definition 3.4.1. Let $\mathbb{K}$ be a field and let $(R, \oplus, \odot)$ be a totally ordered idempotent semiring (i.e., a semiring such that $x \oplus x=x$ for all $x \in R$. A function $v: \mathbb{K} \rightarrow R$ is a valuation on $\mathbb{K}$ if the following hold for elements $x, y \in \mathbb{K}$ :

(1) $v(x)=0_{R}$ iff $x=0_{\mathbb{K}}$,

(2) $v(x \cdot y)=v(x) \odot v(y)$, and

(3) for nonzero $x, y, v(x+y) \geq v(x) \oplus v(y)$, with equality if $v(x) \neq$ $v(y)$ (see Lemma 3.4.7).

The image of a valuation $v$ will be denoted by $\Gamma_{v}$, and is called the value group of $v$.

Definition 3.4.2. Let $\mathbb{K}, R$ and $v$ be as above. Then the set

$$
S=\left\{x \in \mathbb{K} \mid v(x) \geq 0_{R}\right\}
$$

is called the valuation ring of the valuated field $(\mathbb{K}, v)$, and the set

$$
\mathfrak{m}=\left\{x \in \mathbb{K} \mid v(x)>0_{R}\right\}
$$

is its maximal ideal.

We define the residue field $k$ as $S / \mathfrak{m}$, the valuation ring modulo its maximal ideal.

Definition 3.4.3. Let $\mathbb{T}$ be the semiring defined as follows. The elements of $\mathbb{T}$ are the real numbers and infinity $\mathbb{R} \cup\{\infty\}$, with addition $\oplus$ defined as taking the minimum, and multiplication $\odot$ defined as the usual addition. That is, for $x, y \in \mathbb{T}$ we have $x \oplus y=\min (x, y)$ (where $\infty$ is the identity element) and $x \odot y=x+y$. (This is called the "min-plus" convention - there is an isomorphic tropical semiring defined by taking $\mathbb{T}$ as the set containing $\mathbb{R} \cup\{-\infty\}$ and with addition defined as taking the maximum, which is called the "max-plus" convention.)

Clearly $\mathbb{T}$ fulfils all the axioms for a field, except for additive inverses. We call $\mathbb{T}$ the tropical semiring.

Since for any $x \in \mathbb{T}$ we have $x \oplus x=\min \{x, x\}=x$, all elements of $\mathbb{T}$ are idempotent with respect to addition, thus $\mathbb{T}$ is an idempotent semiring.

Example 3.4.4. Let $v$ be the valuation $k \rightarrow \mathbb{T}$ such that $v(x)=0$ for all $0_{k} \neq x \in k$, and $v\left(0_{k}\right)=\infty$. Then we call $v$ the trivial valuation.

Example 3.4.5. Let $\frac{a}{b} \in \mathbb{Q}$, and take some prime integer $p$. We can write $\frac{a}{b}=p^{k} \cdot \frac{c}{d}$ where $c, d$ are nt divisible by $p$. Let $v: \mathbb{Q} \rightarrow \mathbb{Z}$ be the valuation mapping $\frac{a}{b} \mapsto k$. Then we call $v$ the $p$-adic valuation. 
Example 3.4.6. Let $\mathbb{C}\{\{t\}\}$ be the field of Puiseux series with coefficients in $\mathbb{C}$. The field of Puiseux series is the algebraic closure of the field of power series with coefficients in $\mathbb{C}$, and allows the indices of $t$ to be rational as well as integral. We define the $t$-adic valuation on $\sum a_{i} t^{i} \in \mathbb{C}\{\{t\}\}$ to be the lowest $i$ for which $a_{i} \neq 0$. This is the valuation we will be using in most of the following chapters.

For example, the series $5 t^{-3}+(1+2 i) t^{-\frac{3}{2}}$ has valuation -3 under the $t$-adic valuation.

We can also define a valuation on Puiseux series in multiple variables by considering the variables in lexographic (lex), reverse lexographic (rlex), or some other ordering, which produces a vector-valued valuation.

For example, the series $\left(t+2 t^{3}\right) s^{-1}+t s^{2}$ has valuation $(-1,1)$ when we use the lex ordering.

The following lemma will be necessary for the proof of Kapranov's Theorem 3.8.3.

Lemma 3.4.7. Let $v: \mathbb{K} \rightarrow \mathbb{T}$ be a valuation, and suppose $v(a) \neq v(b)$ for some $a, b \in \mathbb{K}$. Then $v(a+b)=v(a) \oplus v(b)$.

Proof. Without loss of generality, we can assume that $v(b)>v(a)$. The third axiom of the definition of a valuation states that $v(a+b) \geq$ $v(a) \oplus v(b)$, which implies that $v(a+b) \geq v(a)$. We will show here that the opposite inequality holds.

From the third axiom again, we have $v(a) \geq v(a+b) \oplus v(-b)$. Now, $1^{2}=1$, which implies that $v(1)=0$, and $(-1)^{2}=1$, so that $v(-1)=0$ also (by axiom (2)). Thus $v(-b)=v(b)$, and we get $v(a) \geq v(a+b) \oplus v(b)$, i.e., $v(a) \geq v(a+b)$, as required.

We will need the following lemma in the proof of Kapranov's Theorem in Section 3.8

Lemma 3.4.8. Let $\mathbb{K}$ be a valued field with a splitting $\Gamma_{v} \rightarrow \mathbb{K}^{*}, w \mapsto t^{w}$, so that the valuation $v\left(t^{w}\right)=w$. Let $\alpha_{1}, \ldots, \alpha_{n} \in \mathbb{K}^{*}$ and $w_{1}, \ldots, w_{n} \in \Gamma_{v}$, and consider the set of all $\boldsymbol{y}=\left(y_{1}, \ldots, y_{n}\right) \in\left(\mathbb{K}^{\star}\right)^{n}$ satisfying $v\left(y_{i}\right)=w_{i}$ and $\overline{t^{-w_{i}} y_{i}}=\alpha_{i}$ for $i=1, \ldots, n$. This set is Zariski dense in $\left(\mathbb{K}^{\star}\right)^{n}$.

Proof. We will show that for any nonzero polynomial $h \in \mathbb{K}\left[x_{1}^{ \pm 1}, \ldots, x_{n}^{ \pm 1}\right]$ there exists a point $\mathbf{y}$ of the desired form with $h(\mathbf{y}) \neq 0$. For each $i$ fix $z_{i} \in R$, where $R$ is the valuation ring, with $\overline{z_{i}}=\alpha_{i}$. Then $y_{i}=t^{w_{i}} z_{i}$ satisfies $v\left(y_{i}\right)=w_{i}$. Since $\alpha_{i} \neq 0$, this implies that $v\left(z_{i}\right)=0$ (by definition of the residue field). Note that each coordinate $y_{i}$ can be replaced by infinitely many other possibilities with the same valuation e.g., $y_{i}+t^{w_{i}+j}$ for any $j>0$.

Now we show by induction on $n$ that we can always choose $\mathbf{y}$ of this form such that $h(\mathbf{y}) \neq 0$. The polynomial $h$ has finitely many roots, so we choose $y_{1}$ from the infinitely many possibilities so that it does not coincide with any of these roots. When $n>1$, we can write 
$h=\sum h_{j} x_{n}^{j}$, where $h_{j} \in \mathbb{K}\left[x_{1}^{ \pm}, \ldots, x_{n-1}^{ \pm}\right]$. By inductive hypothesis, there is some $\mathbf{y}^{\prime}=\left(y_{1}, \ldots, y_{n-1}\right) \in\left(\mathbb{K}^{*}\right)^{n-1}$ with $v\left(y_{i}\right)=w_{i}$ and $\overline{t^{-w_{i}} y_{i}}=\alpha_{i}$, such that $h_{j}\left(\mathbf{y}^{\prime}\right) \neq 0$ for all $j$. Now we choose $y_{n}$ with $v\left(y_{n}\right)=w_{n}$ and $\overline{t^{-w_{n}} y_{n}}=\alpha_{n}$ from the infinite number of possibilities so that it does not coincide with the finitely many roots of $h\left(y_{1}, \ldots, y_{n-1}, x_{n}\right) \in \mathbb{K}\left[x_{n}^{ \pm}\right]$.

3.4.2. Tropicalisation. There are two kinds of tropicalisation we will consider, set-theoretic tropicalisation, which will be covered in this section, and scheme-theoretic tropicalisation, which will be defined and discussed in depth in Section 3.11. We will focus on the case of homogeneous polynomials, since we will be working in projective space in later sections, but there are analogues to all of the following for Laurent polynomials.

Definition 3.4.9. Given a polynomial $p(\mathbf{x})=\sum_{\mathbf{i} \in \mathbb{Z}^{n}} a_{\mathbf{i}} \mathbf{x}^{\mathbf{i}} \in \mathbb{K}\left[x_{0}, \ldots, x_{n}\right]=$ $\mathbb{K}[\mathbf{x}]$, where $\mathbb{K}$ is a field, and a valuation $v: \mathbb{K} \rightarrow \mathbb{T}$, we define its (set theoretic) tropicalisation $\operatorname{trop}(p(\mathbf{x}))$ as follows.

$$
\begin{aligned}
\operatorname{trop}(p(\mathbf{x})) & =\bigoplus_{i=0}^{n} v\left(a_{\mathbf{i}}\right) \otimes \mathbf{x}^{\mathbf{i}} \\
& =\min \left\{v\left(a_{\mathbf{i}}\right)+\sum_{j} i_{j} x_{j}\right\} \in \mathbb{T}[\mathbf{x}] .
\end{aligned}
$$

This gives a piecewise linear function on $\mathbb{T}^{n+1}$. We define the support of $p(\mathbf{x})$ to be the set $\left\{\mathbf{i}: a_{\mathbf{i}} \neq 0\right\}$, denoted by $\operatorname{Supp}(p(\mathbf{x}))$.

We will usually, unless otherwise specified, take $\mathbb{K}$ to be the field of Puiseux series with complex coefficients $\mathbb{C}\{\{t\}\}$ and the valuation to be the $t$-adic valuation as defined in 3.4.6.

Example 3.4.10. Under the trivial valuation, the polynomial $2 x^{3}-$ $4 i x^{2} y+y^{2} \in \mathbb{C}[x, y]$ tropicalises to

$$
\min \{3 x, 2 x+y, 2 y\} .
$$

Example 3.4.11. Under the 2-adic valuation the polynomial $6 x^{2}-$ $\frac{1}{4} x y+18 y^{2} \in \mathbb{Q}[x, y]$ tropicalises to

$$
\min \{2+2 x,-2+x+y, 1+2 y\} .
$$

Example 3.4.12. The polynomial $\left(t^{-1}-1\right) x^{2}+2 t x y \in \mathbb{C}\{\{t\}\}[x, y]$ tropicalises to

$$
\min \{-1+2 x, 1+x+y\} .
$$

Definition 3.4.13. Let $p \in \mathbb{K}\left[x_{0}, \ldots, x_{n}\right]$ be a homogeneous polynomial, and write $p=\sum_{\mathbf{u}} c_{\mathbf{u}} \mathbf{x}^{\mathbf{u}}$, where $\mathbf{x}$ denotes the variables $x_{0}, \ldots, x_{n}$ and $\mathbf{u} \in \mathbb{N}^{n+1}$ is the vector of their exponents. Let $\mathbf{w} \in \mathbb{T}^{n+1}$. We define the generic valuation $v_{\mathbf{w}}(p)$ of $p$ at $\mathbf{w}$ to be

$$
v_{\mathbf{w}}(p)=\operatorname{trop}(p)(\mathbf{w})=\min \left\{v\left(c_{\mathbf{u}}\right)+\mathbf{w} \cdot \mathbf{u}: c_{\mathbf{u}} \neq 0\right\} .
$$


Example 3.4.14. Take, for example, the discriminant of a quadratic $\Delta=B^{2}-4 A C \in \mathbb{C}\{\{t\}\}[A, B, C]$, with the usual valuation on $\mathbb{C}\{\{t\}\}$. Let $\mathbf{w}=(4,3,2)$. The generic valuation of $\Delta$ at $\mathbf{w}$ is therefore

$$
v_{\mathbf{w}}(\Delta)=\min \{2 v(B), v(A)+v(C)\}=\min \{6,6\}=6 .
$$

Remark 3.4.15. Note that the true valuation of a polynomial may differ from the generic valuation. If we take $A=t^{4}, B=2 t^{3}$, and $C=t^{2}+t^{3}$, then $(v(A), v(B), v(C))=(4,3,2)$ and the generic valuation of $B^{2}-4 A C$ with those specific values is as above. However, substituting those values in gives

$$
B^{2}-4 A C=4 t^{6}-4 t^{6}-4 t^{7}=4 t^{7},
$$

the valuation of which is 7 , not 6 .

In the context of this work, we are interested in whether this discrepancy between the generic valuation and the true valuation of the discriminant of a polynomial $p(\mathbf{x})$ is encoded in the tropical ideal it generates, and what information we can glean from that.

3.5. Tropical Initial Forms. In this section we will look at tropical Gröbner bases in the homogeneous case. There exists an analogue of Gröbner bases for tropical Laurent polynomials, but that is beyond the scope of what we need for this work.

Definition 3.5.1. Let $p(\mathbf{x})=\sum_{\mathbf{i} \in \operatorname{Supp}(p)} a_{\mathbf{i}} \mathbf{x}^{\mathbf{i}}$ be a polynomial in $\mathbb{K}\left[x_{0}, \ldots, x_{n}\right]$, $v$ be a valuation $\mathbb{K} \rightarrow S$, and fix some $\mathbf{w} \in S^{n+1}$. Let the initial form $\operatorname{in}_{\mathbf{w}}(p)$ of $p$ with respect to $\mathbf{w}$ is defined as follows.

$$
\operatorname{in}_{\mathbf{w}}(p)=\sum_{\mathbf{j}_{\mathbf{w}}} \overline{t^{-\alpha} a_{\mathbf{j}}} \mathbf{x}^{\mathbf{j}}
$$

where $\alpha$ is the valuation of $a_{\mathbf{j}}$ and $\mathbf{j}_{\mathbf{w}}$ denotes the set of $\mathbf{j} \in \operatorname{Supp}(p)$ such that the minimum of $\operatorname{trop}(p)(\mathbf{w})$ is achieved by $v\left(a_{\mathbf{j}}\right)+\mathbf{j} \cdot \mathbf{w}$.

If $I$ is a homogeneous ideal in $\mathbb{K}\left[x_{0}, \ldots, x_{n}\right]$, we define its initial ideal $\operatorname{in}_{\mathbf{w}}(I)$ to be

$$
\operatorname{in}_{\mathbf{w}}(I):=\left\langle\operatorname{in}_{\mathbf{w}}(f): f \in I\right\rangle \subset k\left[x_{0}, \ldots, x_{n}\right],
$$

where $k$ is the residue field of $\mathbb{K}$ under the valuation $v$. Note that $\operatorname{in}_{\mathbf{w}}(I)$ is an ideal in projective $k$-space (see Lemma 3.5.3 below). We say that a set $\mathcal{G}=\left\{g_{1}, \ldots, g_{k}\right\} \in I$ is a Gröbner basis for $I$ with respect to $\mathbf{w}$ if the initial forms of the $g_{i}$ generate the initial ideal $\operatorname{in}_{\mathrm{w}}(I)$. That is, if

$$
\operatorname{in}_{\mathbf{w}}(I)=\left\langle\operatorname{in}_{\mathbf{w}}\left(g_{1}\right), \ldots, \operatorname{in}_{\mathbf{w}}\left(g_{k}\right)\right\rangle .
$$

Example 3.5.2. Let $I=\langle f\rangle=\left\langle x^{2}+x y+2 z^{2}\right\rangle \subset \mathbb{P}_{\mathbb{C}\{\{t\}\}}^{2}$ be a homogeneous ideal, and fix $\mathbf{w}=(4,4,9)$. We have $\operatorname{trop}(f)=\min \{2 x, x+y, 2 z\}$ and $\operatorname{trop}(f)(\mathbf{w})=\min \{8,8,9\}$. The minimum is achieved in the first and second term, thus $\operatorname{in}_{\mathbf{w}}(f)=x^{2}+x y$. 
Lemma 3.5.3. [Lemma 2.4.2 [17]] Let $I \subset \mathbb{K}\left[x_{0}, \ldots, x_{n}\right]$ be a homogeneous ideal, $v: \mathbb{K} \rightarrow \mathbb{T}$ be a valuation on $\mathbb{K}$, and fix some $\boldsymbol{w} \in$ $\mathbb{T}^{n+1}$. Then $i n_{w}(I)$ is homogeneous, and we may choose a homogeneous Gröbner basis for $I$.

Proof. To show that $\operatorname{in}_{\mathrm{w}}(I)$ is homogeneous, consider some element $f \in \mathbb{K}\left[x_{0}, \ldots, x_{n}\right]$ where $f=\sum_{i \geq 0} f_{i}$, where each $f_{i}$ is homogeneous of degree $i$. The initial form $\operatorname{in}_{\mathbf{w}}(f)$ of $f$ is the sum of the initial forms $\operatorname{in}_{\mathbf{w}}\left(f_{i}\right)$ with $\operatorname{trop}(f)(\mathbf{w})=\operatorname{trop}\left(f_{i}\right)(\mathbf{w})$, i.e., the sum of the initial forms where the minimum is met. Since each $f_{i} \in I$ then $\operatorname{in}_{\mathrm{w}}(I)$ is generated by $\operatorname{in}_{\mathbf{w}}(f)$ with homogeneous $f$. Since the initial form of a homogeneous $f$ must also be homogeneous, the ideal $\operatorname{in}_{\mathbf{w}}(I)$ must be homogeneous also. The polynomial ring is Noetherian, implying that $\operatorname{in}_{\mathrm{w}}(I)$ is finitely generated by the $\operatorname{in}_{\mathbf{w}}(f)$, thus the corresponding $f$ form a homogeneous Gröbner basis for $I$.

In the case of a homogeneous ideal $I$, every Gröbner basis generates $I$ (as is the case in classical Gröbner theory), but it is worth noting that this is not true for ideals in general. For a proof see [2], where Chan and Maclagan develop an algorithm based on Buchberger's algorithm for computing Gröbner bases over valuated fields, and show that tropical Gröbner bases share some important properties with their classical counterparts.

The following lemma will be used in the proof of Theorem 3.8.3.

Lemma 3.5.4. Let $f, g \in \mathbb{K}\left[x_{0}, \ldots, x_{n}\right]$, and let $\boldsymbol{w} \in \Gamma_{v}^{n}$, where $\Gamma_{v}^{n}$ is the value group of $v$. Then

$$
i n_{w}(f g)=i n_{w}(f) i n_{w}(g)
$$

Proof. Let $f=\sum c_{\mathbf{u}} x^{\mathbf{u}}$ and $=\sum d_{\mathbf{u}} x^{\mathbf{u}^{\prime}}$. Then we can write

$$
f \cdot g=\sum e_{\mathbf{v}} x^{\mathbf{v}}
$$

where $e_{\mathbf{v}}=\sum_{\mathbf{u}+\mathbf{u}^{\prime}=\mathbf{v}} c_{\mathbf{u}} d_{\mathbf{u}^{\prime}}$. Now, put $W_{1}=\operatorname{trop}(f)(\mathbf{w})$ and $W_{2}=$ $\operatorname{trop}(g)(\mathbf{w})$. We get

$$
\begin{aligned}
\operatorname{trop}(f g)(\mathbf{w}) & =\min \left\{v\left(e_{\mathbf{v}}\right)+\mathbf{w} \cdot \mathbf{v}: \mathbf{v} \in \mathbb{N}^{n+1}, e_{\mathbf{v}} \neq 0\right\} \\
& =\min \left\{v\left(c_{\mathbf{u}}\right)+v\left(d_{\mathbf{u}}\right)+\mathbf{w} \cdot\left(\mathbf{u}+\mathbf{u}^{\prime}\right)\right\} \\
& =W_{1}+W_{2}
\end{aligned}
$$


Now, we have

$$
\begin{aligned}
& \operatorname{in}_{\mathbf{w}}(f g)=\sum_{\mathbf{v}: v\left(e_{\mathbf{v}}\right)+\mathbf{w} \cdot \mathbf{v}=W_{1}+W_{2}} \overline{e_{\mathbf{v}} t^{-v\left(e_{\mathbf{v}}\right)}} x^{\mathbf{v}} \\
& =\sum_{\mathbf{v}: v\left(e_{\mathbf{v}}\right)+\mathbf{w} \cdot \mathbf{v}=W_{1}+W_{2}} \sum_{\mathbf{u}+\mathbf{u}^{\prime}=\mathbf{v}} \overline{c_{\mathbf{u}} d_{\mathbf{u}^{\prime}} t^{-W_{1}-W_{2}+\mathbf{w} \cdot\left(\mathbf{u}+\mathbf{u}^{\prime}\right)}} x^{\mathbf{v}} \\
& \text { (since } v\left(e_{\mathbf{v}}\right)=W_{1}+W_{2}-\mathbf{w} \cdot \mathbf{v} \text { ) } \\
& =\left(\sum_{\mathbf{u}: v\left(c_{\mathbf{u}}+\mathbf{w} \cdot \mathbf{u}=W_{1}\right.} \overline{c_{\mathbf{u}} t^{-v\left(c_{\mathbf{u}}\right)}} x^{\mathbf{u}}\right) \cdot\left(\sum_{\mathbf{u}^{\prime}: v\left(d_{\mathbf{u}},+\mathbf{w} \cdot \mathbf{u}=W_{1}\right.} \overline{d_{\mathbf{u}^{\prime}} t^{-v\left(d_{\left.\mathbf{u}^{\prime}\right)}\right.} x^{\mathbf{u}^{\prime}}}\right) \\
& =\operatorname{in}_{\mathbf{w}}(f) \operatorname{in}_{\mathbf{w}}(g) \text {. }
\end{aligned}
$$

3.6. Polyhedral Geometry and Newton Polygons. Before we go any further, it will be useful to briefly cover some definitions from polyhedral geometry which we will need later.

Definition 3.6.1. Let $X \subset \mathbb{R}^{n}$ be a subset of $\mathbb{R}^{n}$. We say that the set $X$ is convex if for all $\mathbf{u}, \mathbf{v} \in X$ and $0 \leq \lambda \leq 1$, we have $\lambda \mathbf{u}+(1-\lambda) \mathbf{v} \in X$.

Now let $U \subset \mathbb{R}^{n}$ be a finite set of points $\left\{\mathbf{u}_{1}, \ldots, \mathbf{u}_{r}\right\}$ in $\mathbb{R}^{n}$. We define $\operatorname{Conv}(U)$, the convex hull of $U$, to be the smallest convext set containing $U$. (In $\mathbb{R}^{2}$ we can informally picture this by imagining the points of $U$ as nails in a board, and wrapping a length of string around the outside of them gives the convex hull.) We call the set

$$
\operatorname{Conv}(U)=\left\{\sum_{i=1}^{r} \lambda_{i} \mathbf{u}_{i}: 0 \leq \lambda \leq 1, \sum_{i=1}^{r} \lambda_{i}=1\right\}
$$

a polytope. A two dimensional polytope is called a polygon, and we will restrict our attention to polygons in most of what follows.

If $U$ is a finite set in $\mathbb{R}^{n}$ as above, we can define the positive hull $\operatorname{Pos}(U)$ of $U$ to be the set

$$
\operatorname{Pos}(U):=\left\{\sum_{i=1}^{r} \lambda_{i} \mathbf{u}_{i} \in \mathbb{R}^{n}: \lambda_{i} \geq 0 \forall i\right\} .
$$

We define a polyhedral cone $C$ in $\mathbb{R}^{n}$ of a finite subset $U \subset \mathbb{R}^{n}$ to be $\operatorname{Pos}(U)$. A cone $C$ is called simplicial if the $\mathbf{u}_{i}$ are linearly independent.

A polyhedral fan $\mathcal{F}$ is a collection of cones $C_{i}$ with the following two properties:

(1) If $C_{i} \in \mathcal{F}$, then every face of $C_{i}$ is also in $\mathcal{F}$.

(2) If $C_{1}, C_{2} \in \mathcal{F}$, then $C_{1} \cap C_{2}$ is a face of both $C_{1}$ and $C_{2}$.

Definition 3.6.2. A polyhedron $P$ can be defined as an intersection of finitely many closed half-spaces in $\mathbb{R}^{n}$; more precisely,

$$
P=\left\{\mathrm{x} \in \mathbb{R}^{n}: A \mathrm{x} \leq \mathbf{b}\right\},
$$


where $A$ is a $d \times n$-matrix and $\mathbf{b} \in \mathbb{R}^{d}$.

As a simple example, take the unit square $U$ in $\mathbb{R}^{2}$ with vertices $(0,0)$, $(0,1),(1,0)$, and $(1,1) . U$ is the intersection of the half planes $x \leq 1$, $x \geq 0, y \leq 1$, and $y \geq 0$. We thus have

$$
U=\left\{(x, y) \in \mathbb{R}^{2}: A \cdot(x, y) \leq \mathbf{b}\right\},
$$

where

$$
A=\left[\begin{array}{cc}
1 & 0 \\
0 & 1 \\
-1 & 0 \\
0 & -1
\end{array}\right], \text { and } \mathbf{b}=\left[\begin{array}{l}
1 \\
1 \\
0 \\
0
\end{array}\right]
$$

A face of a polyhedron $P$ is defined to be the intersection of $P$ with any closed halfspace, whose boundary is disjoint from the interior of $P$. If $P$ is an $n$-dimensional polyhedron, a facet of $P$ is defined to be a face of dimension $(n-1)$.

A polyhedral complex is defined as a collection $\Sigma$ of polyhedra such that

(1) if $P \in \Sigma$ then so is any face of $P$.

(2) if $P, Q \in \Sigma$ then either $P \cap Q=\emptyset$ or $P \cap Q$ is a face of both $P$ and $Q$.

We say that a polyhedron $P=\left\{\mathbf{x} \in \mathbb{R}^{n}: A \mathbf{x} \leq \mathbf{b}\right\}$ is $\Gamma$-rational if the $d \times n$-matrix $A$ has entries in $\mathbb{Q}$ and $\mathbf{b} \in \Gamma^{d} \subset \mathbb{R}^{d}$. A polyhedral complex $\Sigma$ is said to be $\Gamma$-rational if all of the polyhedra contained in $\Sigma$ are $\Gamma$-rational.

The lineality space of a polyhedron $P$ is defined as the largest linear subspace $V \subset \mathbb{R}^{n}$ such that $\mathbf{x} \in P$ and $\mathbf{v} \in V$ implies that $\mathbf{x}+\mathbf{v} \in P$.

For example, the lineality space of the unit square $U$ from above is simply $\{(0,0)\}$, since any subspace of $\mathbb{R}^{2}$ containing $\left(\begin{array}{l}1 \\ 0\end{array}\right)$ or $\left(\begin{array}{l}0 \\ 1\end{array}\right)$ would easily result in $\mathbf{x}+\mathbf{v} \notin U$ in general (e.g. take $\mathbf{v}$ to be $\left(\begin{array}{l}8 \\ 0\end{array}\right)$ or $\left(\begin{array}{l}0 \\ 8\end{array}\right)$ respectively).

Definition 3.6.3. Let $R=\mathbb{K}\left[x_{1}, \ldots, x_{n}\right]$. Given

$$
f=\sum_{\mathbf{u} \in \mathbb{N}^{n}} c_{\mathbf{u}} \mathbf{x}^{\mathbf{u}} \in R
$$

the Newton polytope of $f$, Newt $(f)$, is given by

$$
\operatorname{Conv}\left(\mathbf{u}: c_{\mathbf{u}} \neq 0\right) \subset \mathbb{R}^{n} .
$$

In 2-dimensional space, we call Newt $(f)$ the Newton polygon of $f$.

A marked polygon for our purposes is the Newton polygon of a polynomial $f$ with the integer lattice points marked in. We will denote a marked polygon by $(Q, A)$, where $Q$ is a polygon and $A$ denotes the set of integer lattice points contained within $Q$.

A marked subdivision of $(Q, A)$ is a finite family of marked polygons $\left\{\left(Q_{i}, A_{i}\right) \mid i=1, \ldots, k\right\}$ with the following properties:

(1) $\left(Q_{i}, A_{i}\right)$ is a (2-dimensional) marked polygon for each $i$, 
(2) $Q=\cup_{i=1}^{k}$ is a subdivision of $Q$, i.e., $Q_{i} \cap Q_{j}$ is a face (possibly empty) of $Q_{i}$ and $Q_{j}$ for all $i, j$,

(3) $A_{i} \subset A$ for all $i$, and

(4) $A_{i} \cap\left(Q_{i} \cap Q_{j}\right)=A_{j} \cap\left(Q_{i} \cap Q_{j}\right)$ for all $i, j$.

A marked subdivision is called a triangulation if for all $i, Q_{i}$ is a simplex.

We will use the following example to demonstrate the duality between marked polytopes and tropical curves in $\mathbb{R}^{2}$.

Example 3.6.4. Consider the polynomial $f=x^{2}+y^{2}+t y+t^{2} x \in \mathbb{A}_{\mathbb{C}\{\{t\}\}}^{2}$. The exponents correspond to the points $(0,2),(0,1),(1,0)$ and $(2,0)$, and their convex hull is shown in the picture below.

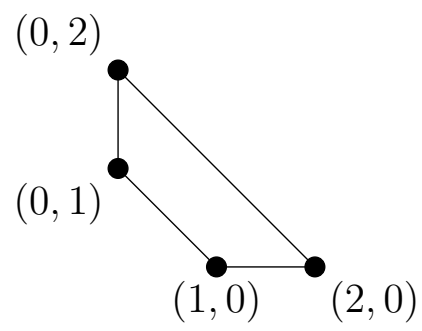

We will now construct the marked subdivision of the above polytope that is dual to the tropical hypersurface $\operatorname{trop}(f)$. The weight vector $\mathbf{w}$ associated to $f$ is $\mathbf{w}=(0,0,1,2)$. We take the set of points

$$
\{(2,0,0),(0,2,0),(0,1,1),(1,0,2)\}
$$

in $\mathbb{R}^{3}$ and take the convex hull. The lower faces are the ones whose inner normal vector (i.e., the normal vector in the direction of the inside of the polytope) has positive last coordinate. The lower faces are shown in the diagram below.

These lower faces project onto the Newton polygon to form the following marked subdivision. See the diagram in Example 3.7.1 which shows the tropical variety $\operatorname{trop}(f)$.

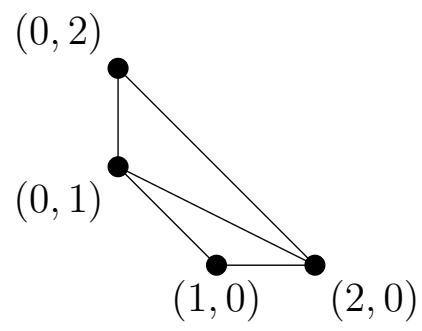

3.7. The Gröbner Complex. A full description of Gröbner complexes and their construction can be found in Chapter 2.5 of [17]. The Gröbner complex is the ambient space in which a tropical variety lives. Here we will just work through an example to give the general idea. 
Example 3.7.1. Consider the ideal

$$
I=\langle f(x, y)\rangle=\left\langle x^{2}+y^{2}+t y+t^{2} x\right\rangle \subset \mathbb{A}_{\mathbb{C}\{\{t\}\}}^{2} .
$$

We have

$$
\operatorname{trop}(f)=\min \{2 x, 2 y, 1+y, 2+x\} .
$$

The Gröbner complex of $I$ is a polyhedral complex whose cells correspond to the initial ideals of $I$, and shows where the map $\operatorname{trop}(f)$ is linear. As we vary $\mathbf{w}$ over $\mathbb{R}^{2}$ we get the following initial ideals:

\begin{tabular}{|c|c|c|c|}
\hline & $\mathbf{w}$ & $\operatorname{trop}(f)(\mathbf{w})$ & $\operatorname{in}_{\mathbf{w}}(f)$ \\
\hline 1 & $(0,1)$ & $\min \{0,2,2,2\}$ & $\left\langle x^{2}\right\rangle$ \\
2 & $(1,0)$ & $\min \{2,0,1,3\}$ & $\left\langle y^{2}\right\rangle$ \\
3 & $(2,2)$ & $\min \{4,4,3,4\}$ & $\langle y\rangle$ \\
4 & $(3,5)$ & $\min \{6,10,6,5\}$ & $\langle x\rangle$ \\
5 & $(0,0)$ & $\min \{0,0,1,2\}$ & $\left\langle x^{2}+y^{2}\right\rangle$ \\
6 & $\left(\frac{3}{2}, 2\right)$ & $\min \{3,4,3,4\}$ & $\left\langle x^{2}+y\right\rangle$ \\
7 & $(2,5)$ & $\min \{4,10,6,4\}$ & $\left\langle x^{2}+x\right\rangle$ \\
8 & $(2,1)$ & $\min \{4,2,2,4\}$ & $\left\langle y^{2}+y\right\rangle$ \\
9 & $(3,4)$ & $\min \{6,8,5,5\}$ & $\langle y+x\rangle$ \\
10 & $(2,3)$ & $\min \{4,6,4,4\}$ & $\left\langle x^{2}+y+x\right\rangle$ \\
11 & $(1,1)$ & $\min \{2,2,2,3\}$ & $\left\langle x^{2}+y^{2}+y\right\rangle$ \\
\hline
\end{tabular}

The diagram below shows the Gröbner complex, with each cell labelled according to the numbers in the table.

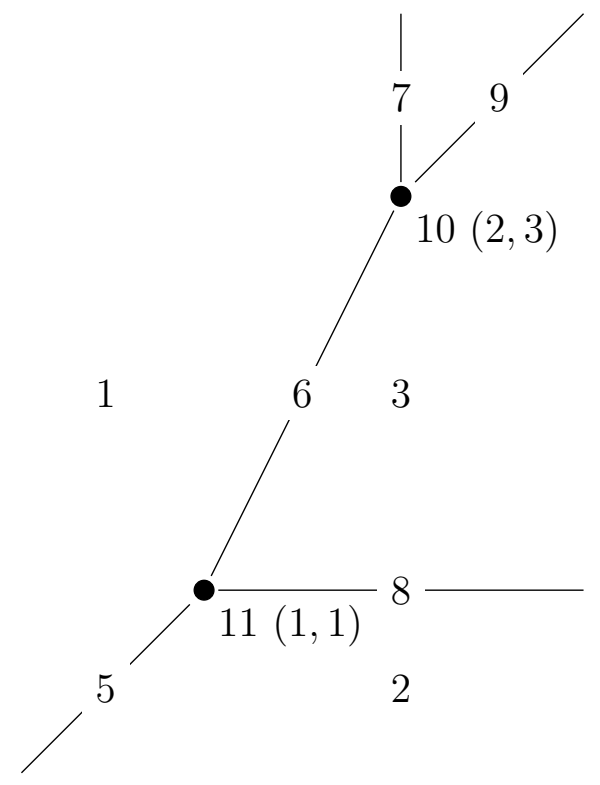

The monomial initial ideals correspond to the 2-dimensional cells. The binomial initial ideals correspond to the 1-dimensional cells at the intersections of the 2-dimensional cells. For example, cell 5 corresponds to the intersection of cells 1 and 2. Finally, the trinomial initial ideals correspond to the two 1-dimensional cells at $(1,1)$ and $(2,3)$. 
We note here that because our ideal $I$ is generated by a single polynomial, the Gröbner complex and the tropical variety are the same, but for general tropical varieties this isn't true.

Comparing the tropical variety of $f$ to the triangulation in Example 3.6.4, we see that 10 and 11 correspond to the 2 -simplices, 5, 6, 7, 8 and 9 correspond to the 1-simplices, and 1, 2, 3 and 4 correspond to the 0 -simplices.

3.8. Kapranov's Theorem and the Fundamental Theorem of Tropical Algebraic Geometry. Recall that if $p(\mathbf{x}) \in \mathbb{K}\left[x_{0}, \ldots, x_{n}\right]$ is a homogeneous polynomial and $\mathbb{K}$ is an algebraically closed field, then its classical variety $V(p)$ is the hypersurface

$$
V(p)=\left\{\mathbf{v} \in \mathbb{K}^{n+1}: p(\mathbf{v})=0\right\}
$$

The tropical hypersurface associated with $p$ is defined as follows.

Definition 3.8.1. The projective tropical hypersurface trop $(V(p))$ is the set

$\left\{w \in \mathbb{P}_{\mathbb{T}}^{n}:\right.$ the minimum in $\operatorname{trop}(p)$ is achieved at least twice $\}$.

There are corresponding definitions for affine varieties and toric varieties, but we will not state them here. A tropical polynomial is a piecewise linear function; the associated tropical hypersurface is the locus where it fails to be linear, i.e., where the minimum is achieved at least twice. If the minimum valuation isn't achieved at least twice, then the terms in $p$ cannot cancel.

Example 3.8.2. Let $p(x)=2 t^{2} x^{2}+x y+t y^{2}+8 \in \mathbb{C}\{\{t\}\}\left[x_{1}, \ldots, x_{n}\right]$. We have $\operatorname{trop}(p)=\min \{2+2 x, x+y, 1+2 y, 0\}$. The tropical hypersurface consists of all values of $x$ and $y$ for which the minimum is met at least twice.

(1) Suppose $2+2 x=x+y$, then $y=x+2$.

(2) Suppose $2+2 x=1+2 y$, then $y=x+\frac{1}{2}$.

(3) Suppose $2+2 x=0$, then $x=-1$.

(4) Suppose $x+y=1+2 y$, then $y=x-1$.

(5) Suppose $x+y=0$, then $y=-x$.

(6) Suppose $1+2 y=0$, then $y=\frac{-1}{2}$.

Now, (1), (3) and (5) meet at the point $(-1,1)$, and (3), (4) and (6) meet at the point $\left(\frac{1}{2}, \frac{-1}{2}\right)$. So the tropical variety is as follows: 


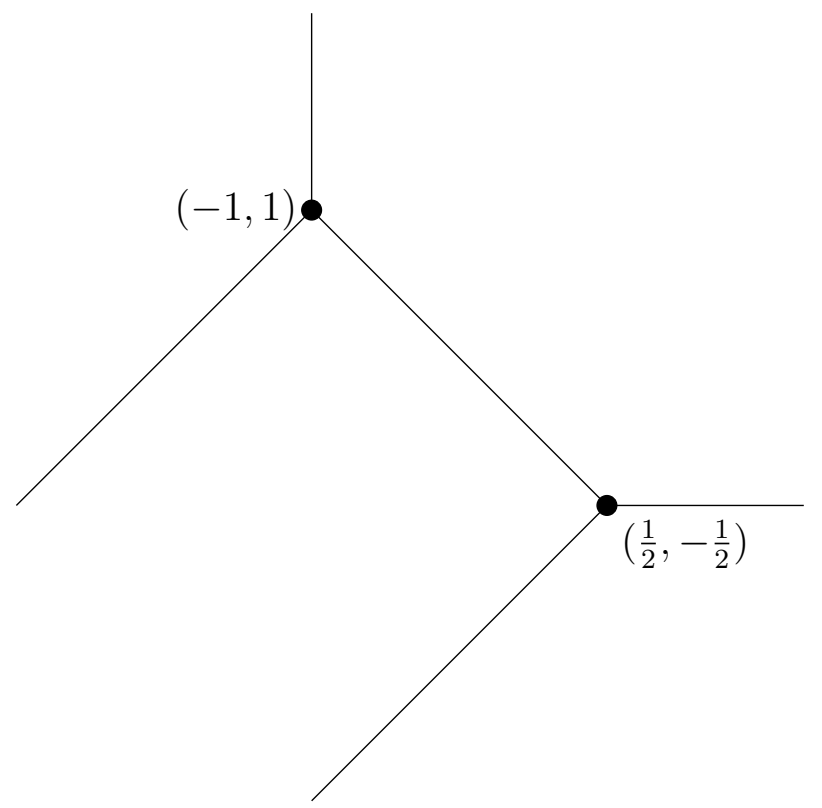

Theorem 3.8.3 (Kapranov's Theorem). Fix a homogeneous polynomial $p(\boldsymbol{x})=\sum_{\boldsymbol{u} \in \mathbb{Z}^{n+1}} c_{\boldsymbol{u}} \boldsymbol{x}^{u} \in \mathbb{K}\left[x_{0}, \ldots, x_{n}\right]$. Let $v: \mathbb{K} \rightarrow \mathbb{T}$ be a non-trivial valuation on $\mathbb{K}$. The following three sets coincide:

(1) the tropical hypersurface trop $(V(p))$ in $\mathbb{P}_{\mathbb{T}}^{n}$;

(2) the closure in $\mathbb{P}_{\mathbb{T}}^{n}$ of the set of $\boldsymbol{w} \in S$ such that in $n_{\boldsymbol{w}}(p)$ is not a monomial;

(3) the closure of the set $\left\{\left[v\left(v_{0}\right): \ldots: v\left(v_{n}\right)\right]: \boldsymbol{v}=\left[v_{0}: \ldots: v_{n}\right] \in\right.$ $V(p)\}$.

Proof. First we will show that $(1)=(2)$. Suppose that $\mathbf{w} \in \operatorname{trop}(V(p))$, then by definition the minimum of $\operatorname{trop}(p)(\mathbf{w})$ is achieved at least twice, and thus $\operatorname{in}_{\mathbf{w}}(p)$ will be a sum of at least two terms, i.e., not a monomial. Conversely, if $\operatorname{in}_{\mathbf{w}}(p)$ is a sum of at least two terms, then that implies the minimum of $\operatorname{trop}(p)(\mathbf{w})$ is achieved at least twice and therefore $\mathbf{w} \in \operatorname{trop}(V(p))$.

Now let us show that $(3) \subset(1)$. Since set (1) is Zariski closed, it suffices to consider points in (3) of the form $v(y):=\left[v\left(y_{0}\right): \ldots: v\left(y_{n}\right)\right]$, where $y:=\left[y_{0}: \ldots: y_{n}\right] \in \mathbb{P}_{\mathbb{K}}^{n}$ satisfies $p(y)=0$. Thus we have

$$
v(p(y))=v(0)=\infty>v\left(c_{\mathbf{u}} y^{\mathbf{u}}\right)
$$

for all $\mathbf{u}$ with $c_{\mathbf{u}} \neq 0$. By Lemma 3.4.7, if $v(a) \neq v(b)$, then $v(a+b)=$ $\min \{v(a), v(b)\}$, and this therefore implies that at least two of the $c_{\mathbf{u}} y^{\mathbf{u}}$ have the same valuation and that the minimum of $v\left(c_{\mathbf{u}} y^{\mathbf{u}}\right.$ is met at least twice. Thus $v(y) \in \operatorname{trop}(V(p))$.

We will prove the final inclusion using the following Proposition, which asserts that every zero of an initial form lifts to a zero of the given polynomial. 
Proposition 3.8.4. Fix $f \in \mathbb{K}\left[x_{0}, \ldots, x_{n}\right]$ and $\boldsymbol{w} \in \Gamma_{v}^{n+1}$ (the valuation group of $v)$. Suppose $i n_{w}(f)$ is not a monomial, and that $\alpha=\left[\alpha_{0}\right.$ : $\left.\ldots: \alpha_{n}\right] \in \mathbb{P}_{\mathbb{K}}^{n}$ satisfies $i_{w}(f)(\alpha)=0$, i.e., $\alpha$ is a zero of the initial form. Then there exists $\boldsymbol{y} \in \mathbb{K}^{n+1}$ satisfying $f(\boldsymbol{y})=0, v(\boldsymbol{y})=\boldsymbol{w}$, and $\overline{t^{-w_{i}} y_{i}}=\alpha_{i}$ for $0 \leq i \leq n$, i.e., $\boldsymbol{y}$ is a zero of $f$ and valuates to $\boldsymbol{w}$.

Proof. We prove the statement using induction on $n$. Using the change of variables $x_{i}^{\prime}=\frac{x_{i}}{x_{0}}$ allows us to work with affine polynomials in this proof. Let us consider the base case $n=1$. Up to multiplication by a unit, we have

$$
f=\sum_{i=0}^{s} c_{i} x^{i}=\prod_{j=1}^{s}\left(a_{j} x-b_{j}\right),
$$

where $c_{0}, c_{s} \neq 0$. By Lemma 3.5.4 $\operatorname{in}_{\mathbf{w}}(f g)=\operatorname{in}_{\mathbf{w}}(f) \operatorname{in}_{\mathbf{w}}(g)$, so that

$$
\operatorname{in}_{\mathbf{w}}(f)=\prod_{j=1}^{s} \operatorname{in}_{\mathbf{w}}\left(a_{j} x-b_{j}\right) .
$$

Suppose $\alpha \in k^{*}$ (i.e., $\alpha$ is non-zero and in the residue field), and $\operatorname{in}_{\mathbf{w}}(f)(\alpha)=0$. Since $\alpha$ is non-zero, this implies that $\operatorname{in}_{\mathbf{w}}(f)$ is not a monomial, and that $\operatorname{in}_{\mathbf{w}}\left(a_{j} x-b_{j}\right)=0$ for some $j$. This in turn implies that $\operatorname{in}_{\mathbf{w}}\left(a_{j} x-b_{j}\right)$ is not a monomial, i.e., $\operatorname{in}_{\mathbf{w}}\left(a_{j} x-b_{j}\right)=a_{j} x-b_{j}$. Therefore $a_{j} w=b_{j}$, and $v\left(a_{j}\right)+w=v\left(b_{j}\right)$. Write $\alpha=\overline{t^{-w} \frac{b_{j}}{a_{j}}}$, and set $y=\frac{b_{j}}{a_{j}}$. Then $f(y)=0, v(y)=w$, and $\overline{t^{-v(y)} y}=\alpha$ as required.

Now we assume $n>1$ and the proposition holds for all $d$ with $1 \leq d \leq n-1$. First suppose that no two monomials in $f$ are divisible by the same power of $x_{n}$, and regard $f$ as a polynomial in $x_{n}$ with coefficients in $\mathbb{K}\left[x_{1}, \ldots, x_{n-1}\right]$. Then the coefficients are all monomials of the form $c x^{\mathbf{u}}$ for $c \in \mathbb{K}, \mathbf{u} \in \mathbb{Z}^{n-1}$.

For the cases where the above doesn't hold, consider the following automorphism. For $l \in \mathbb{N}$, let $\phi_{l}^{*}: \mathbb{K}\left[x_{1}, \ldots, x_{n}\right]$ be given by $\phi_{l}^{*}\left(x_{j}\right)=$ $x_{j} x_{n}^{l^{j}}$ for $1 \leq j \leq n-1$ and $\phi_{l}^{*}\left(x_{n}\right)=x_{n}$. For $\mathbf{u} \in \mathbb{Z}^{n-1}$, we have

$$
\phi_{l}^{*}\left(x^{\mathbf{u}} x_{n}^{i}\right)=x^{\mathbf{u}} x_{n}^{i+\sum_{j=1}^{n-1} u_{j} l^{j}} .
$$

For example, take $x_{1}^{2} x_{2}^{4} x_{3}^{2} \in \mathbb{K}\left[x_{1}, x_{2}, x_{3}\right]$. We get

$$
\phi_{l}^{*}\left(x_{1}^{2} x_{2}^{4} x_{3}^{2}\right)=x_{1}^{2} x_{3}^{2 l} x_{2}^{4} x_{3}^{4 l^{3}} x_{3}^{2}=x_{1}^{2} x_{2}^{4} x_{3}^{2+2 l+4 l^{3}} .
$$

For large $l>>0$ each monomial in $\phi_{l}^{*}(f)$ is divisible by a different power of $x_{n}$.

Now suppose that $\mathbf{y}=\left(y_{1}, \ldots, y_{n}\right) \in \mathbb{K}$ satisfies $\phi_{l}^{*}(f)(\mathbf{y})=0, v\left(y_{i}\right)=$ $w_{i}-l^{i} w_{n}$, and $\overline{t^{-w_{i}+l^{i} w_{n}} y_{i}}=\alpha_{i} \alpha_{n}^{-l^{i}}$ for $1 \leq i \leq n-1$, as well as $v\left(y_{n}\right)=$ $w_{n}$ and $\overline{t^{-w_{n}} y_{n}}=\alpha_{n}$. Define $\mathbf{y}^{\prime} \in \mathbb{K}$ by $y_{i}^{\prime}=y_{i} y_{n}^{l^{i}}$ for $1 \leq i \leq n-1$ and $y_{n}^{\prime}=y_{n}$. Then we get $f\left(\mathbf{y}^{\prime}\right)=0, v\left(\mathbf{y}^{\prime}\right)=\mathbf{w}$ and $\overline{t^{-w_{i}} y_{i}^{\prime}}=\alpha_{i}$. This means that it suffices to prove the statement for $\phi_{l}^{*}(f)$. 
We can thus assume that $f$ can be regarded as a polynomial in $x_{n}$ with coefficients in $\mathbb{K}\left[x_{1}, \ldots, x_{n-1}\right]$. Consider the set of all $\left(y_{1}, \ldots, y_{n-1}\right)$ in $\mathbb{K}^{n-1}$ such that $v\left(y_{i}\right)=w_{i}$ and $\overline{t^{-w_{i}} y_{i}}=\alpha_{i}$ for $1 \leq i \leq n-1$. By Lemma 3.4 .8 this set is Zariski dense in $\mathbb{K}^{n-1}$. Moreover, for all such choices of $y_{i}$, we have $g\left(x_{n}\right)=f\left(y_{1}, \ldots, y_{n-1}, x_{n}\right) \neq 0$.

Write $\mathbf{u}^{\prime}$ for the projection of $\mathbf{u} \in \mathbb{Z}^{n}$ onto the first $n-1$ coordinates (i.e., $\left.\left(u_{1}, \ldots, u_{n-1}, u_{n}\right) \mapsto\left(u_{1}, \ldots, u_{n-1}\right)\right)$. If we write $g=\sum d_{i} x_{n}^{i}$, we have $d_{i}=c_{\mathbf{u}} \mathbf{y}^{\mathbf{u}^{\prime}}$ for a unique $\mathbf{u} \in \mathbb{Z}^{n}$ with $u_{n}=i$. Note that

$$
\begin{aligned}
v\left(d_{i}\right)+w_{n} i & =v\left(c_{\mathbf{u}}\right)+v\left(\mathbf{y}^{\mathbf{u}^{\prime}}\right)+w_{n} i \\
& =v\left(c_{\mathbf{u}}\right)+\mathbf{w}^{\prime} \cdot \mathbf{u}^{\prime}+w_{n} u_{n} \\
& =v\left(c_{\mathbf{u}}\right)+\mathbf{w} \cdot \mathbf{u} .
\end{aligned}
$$

Thus $\operatorname{trop}(g)\left(w_{n}\right)=\operatorname{trop}(f)(\mathbf{w})$ and $\operatorname{in}_{w_{n}}(g)=\operatorname{in}_{\mathbf{w}}(f)\left(\alpha_{1}, \ldots, \alpha_{n-1}, x_{n}\right)$, as shown below.

$$
\begin{aligned}
\operatorname{in}_{w_{n}}(g)= & \sum_{i: v\left(d_{i}\right)+w_{n} i=\operatorname{trop}(g)\left(w_{n}\right)} \overline{t^{-v\left(d_{i}\right)} d_{i}} x_{n}^{i} \\
= & \sum_{\mathbf{u}: v\left(c_{\mathbf{u}} \mathbf{y}^{\mathbf{u}^{\prime}}\right)+w_{n} u_{n}=\operatorname{trop}(g)\left(w_{n}\right)} \overline{t^{-v\left(c_{\mathbf{u}}\right)} c_{\mathbf{u}} t^{-\mathbf{u}^{\prime} \cdot \mathbf{w}^{\prime}} y^{\mathbf{u}^{\prime}}} x_{n}^{u_{n}} \\
= & \sum_{\mathbf{u}: v\left(c_{\mathbf{u}}\right)+\mathbf{w} \cdot \mathbf{u}=\operatorname{trop}(f)(\mathbf{w})} \\
= & \operatorname{in}_{\mathbf{w}}(f)\left(\alpha_{1}, \ldots, \alpha_{n-1}, x_{n}\right) .
\end{aligned}
$$

Thus $\operatorname{in}_{w_{n}}(g)\left(\alpha_{n}\right)=0$. By the $n=1$ base case there is some $y_{n} \in \mathbb{K}^{*}$ with $v\left(y_{n}\right)=w_{n}$ and $\overline{t^{-w_{n}} y_{n}}=\alpha_{n}$ for which $g\left(y_{n}\right)=0$, and therefore $f\left(y_{1}, \ldots, y_{n-1}, y_{n}\right)=0$. We can thus conclude that $\mathbf{y}=\left(y_{1}, \ldots, y_{n}\right)$ is the required point in $V(f)$.

Finally, we show that if $f$ is irreducible, then the set $\mathcal{Y}$ of $\mathbf{y}=$ $\left(y_{1}, \ldots, y_{n}\right)$ with $v(\mathbf{y})=\mathbf{w}$ and $\overline{t^{-w_{i}} y_{i}}=\alpha_{i}$ for $1 \leq i \leq n$ is Zariski dense in $V(f)$. In the previous part of the proof, for any $\left(y_{1}, \ldots, y_{n-1}\right)$ in $\mathbb{K}^{n-1}$ with $v\left(y_{i}\right)=w_{i}$ and $\overline{t^{-w_{i}} y_{i}}=\alpha_{i}$, we constructed a point $\mathbf{y}=\left(y_{1}, \ldots, y_{n-1}, y_{n}\right) \in \mathcal{Y}$. By Lemma 3.4.8, the set of $\left(y_{1}, \ldots, y_{n-1}\right)$ is Zariski dense in $\mathbb{K}^{n-1}$, and therefore the projection of $\mathcal{Y}$ onto the first $n-1$ coordinates is not contained in any hypersurface of $\mathbb{K}^{n-1}$ (if it were, then it could not be dense in $\mathbb{K}^{n-1}$ ).

Consider any polynomial $g \in \mathbb{K}\left[x_{1}, \ldots, x_{n}\right]$ such that $g(\mathbf{y})=0$ for all $\mathbf{y} \in \mathcal{Y}$. Then $\langle f, g\rangle \cap \mathbb{K}\left[x_{1}, \ldots, x_{n}\right]=\{0\}$. The irreducibility of $f$ implies that $g$ must be a multiple of $f$. Therefore $\mathcal{Y}$ is Zariski dense in $V(f)$.

With that, we have proved the "hard part" of Kapranov's Theorem.

The fundamental theorem generalises Kapranov's theorem to arbitrary tropical varieties, but before we can state it, we must define exactly what an arbitrary tropical variety is. We will restrict our attention to projective varieties, although an analogous definition holds for varieties 
in the algebraic torus $\left(\mathbb{K}^{\star}\right)^{n}$. We will not prove the fundamental theorem here; the proof can be found in Chapter 3.2 of [17].

Definition 3.8.5. Let $I$ be a homogeneous ideal in the ring $\mathbb{K}\left[x_{0}, \ldots, x_{n}\right]$ and let $V(I)$ be its variety in projective $n$-space $\mathbb{P}^{n}$. We define the tropicalisation $\operatorname{trop}(V(I))$ to be the intersection of all tropical hypersurfaces defined by homogeneous polynomials in $I$, i.e.,

$$
\operatorname{trop}(V(I))=\cap_{f \in I} \operatorname{trop}(V(f)) .
$$

Note that it is not enough to take the intersection of hypersurfaces where $f$ runs over a generating set of $I$. Instead, we must take $f$ to run over what is called a tropical basis of the ideal (see Chapter X of [17]).

Theorem 3.8.6 (Fundamental Theorem of Tropical Algebraic Geometry). Let $I$ be a homogeneous ideal in $\mathbb{K}\left[x_{0}, \ldots, x_{n}\right]$ and let $V(I)$ be its variety in projective $n$-space $\mathbb{P}^{n}$. Then the following three subsets of $\mathbb{P}_{\mathbb{T}}^{n}$ coincide:

(1) The tropical variety $\operatorname{trop}(V(I))=\cap_{f \in I} \operatorname{trop}(V(f))$;

(2) the closure in $\mathbb{P}_{\mathbb{T}}^{n}$ of the set of all vectors $\boldsymbol{w} \in\left(\Gamma_{v}\right)^{n}$ with in $n_{\boldsymbol{w}}(I)$ not a monomial;

(3) the closure in $\mathbb{P}_{\mathbb{T}}^{n}$ of the set of coordinatewise valuations of points in $V(I)$, i.e.,

$$
v(V(I))=\left\{\left(v\left(u_{1}\right), \ldots, v\left(u_{n}\right)\right):\left(u_{1}, \ldots, u_{n}\right) \in V(I)\right\} .
$$

3.9. Valuated Matroids. Valuated matroids are a generalisation of matroids, objects which provide a useful link between linear algebra and combinatorics. We will give a brief definition of matroids, before moving on to valuated matroids, which have analogous applications in tropical geometry.

Definition 3.9.1. A matroid consists of a set $E$ and the set $\mathcal{I}$ of independent subsets of $E$. The elements of $\mathcal{I}$ have the following properties:

(1) $\mathcal{I}$ is non-empty;

(2) every subset of every element of $\mathcal{I}$ is also in $\mathcal{I}$; and

(3) if $X, Y \in \mathcal{I}$ and $|X|=|Y|+1$, then there is an element $x \in X-Y$ such that $Y \cup\{x\} \in \mathcal{I}$.

A matroid can be described either in terms of its bases, i.e., its maximal (by inclusion) independent subsets, or its set of circuits $\mathcal{C}$, i.e., the minimal dependent subsets of $E$.

We can reformulate the definition of a matroid in terms of $\mathcal{C}$ as follows:

(1) $\emptyset \notin \mathcal{C}$;

(2) there are no $C_{1}, C_{2} \in \mathcal{C}$ such that $C_{1}$ is a proper subset of $C_{2}$; and

(3) if $C_{1}, C_{2}$ are distinct members of $\mathcal{C}$ and $c \in C_{1} \cap C_{2}$, then $\left(C_{1} \cup C_{2}\right)-\{c\}$ contains an element of $\mathcal{C}$. 
Example 3.9.2. Let $\mathcal{M}$ be a matroid such that $E=\{1,2,3,4,5\}$, and $\mathcal{I}$ is the set of all subsets of $E$ containing 3 or fewer elements (e.g., $\{1,3,5\},\{2,3\}$, and $\{4\}$ are all contained in $\mathcal{I})$. Then we say that $\mathcal{M}$ is the uniform matroid of rank 3 on 5 elements. It's clear that all of the above axioms hold, that the bases are all subsets of rank 3, and that any subset containing 4 elements is a circuit.

Example 3.9.3. Let $M$ be a matrix with $m$ rows and $n$ columns. $M$ gives rise to a matroid $\mathcal{M}$ in the following way: if we take the ground set $E$ to be the set of columns of $M$, then the circuits of $\mathcal{M}$ are the subsets of $E$ which are linearly dependent.

Example 3.9.4. Consider the graph below:

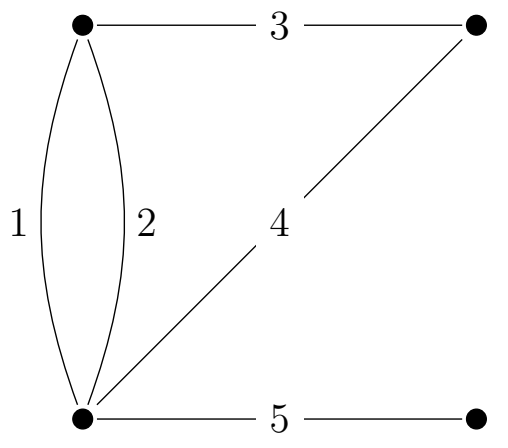

To construct a matroid $\mathcal{M}$ from this graph, we take the edges to be the set $E$, and the independent sets to be all subsets of the edges not containing a cycle. The bases of $\mathcal{M}$ are

$$
\{\{1,3,5\},\{1,4,5\},\{2,3,5\},\{2,4,5\},\{3,4,5\}\},
$$

thus it is non-empty and clearly every subset of the bases must also contain no cycle. For the third axiom, take for example the two independent sets $X=\{2,3,5\}$ and $Y=\{3,4\}$. We have $X \backslash Y=\{2,5\}$, so that $Y \cup\{2\}=\{2,3,4\} \in \mathcal{I}$ and $Y \cup\{5\}=\{3,4,5\} \in \mathcal{I}$.

Now, we define the set $\mathcal{C}$ of circuits as the minimal subsets of $E$ containing cycles, so that the circuits of $\mathcal{M}$ are $\{\{1,2\},\{1,3,4\},\{2,3,4\}\}$. Clearly the empty set is not in $\mathcal{C}$, and no element of $\mathcal{C}$ is a subset of the other. For the third axiom, take for example $(\{1,2\} \cup\{1,3,4\}) \backslash\{1\}=$ $\{2,3,4\} \in \mathcal{C}$.

Example 3.9.5. More generally, we can take a graph $G$ and construct a matroid $\mathcal{M}(G)$ on the set of edges of $G$ by taking the circuits to be the subsets of edges containing a cycle. Any matroid which is isomorphic to a matroid of this form is called a graphic matroid.

Example 3.9.6. Let $E$ be the set $\{1,2,3,4\}$ and let the bases of a matroid $\mathcal{M}$ be all subsets of cardinality 3, i.e.,

$$
\{\{1,2,3\},\{1,2,4\},\{1,3,4\},\{2,3,4\}\} \text {. }
$$

Then we say that $\mathcal{M}$ is the uniform matroid of rank 3 on $E$. 
Definition 3.9.7. Let $S$ be a finite set and $\mathbb{T}$ be the tropical semifield (although this definition holds for any totally-ordered idempotent semiring). Let $d \in \mathbb{N}$, and denote the set of subsets of $S$ of cardinality $d$ by $\mathcal{P}_{d}$. A valuated matroid $\mathcal{M}_{v}$ on $S$ over $\mathbb{T}$ of rank $d$ is a function

$$
v: \mathcal{P}_{d} \rightarrow \mathbb{T}
$$

that satisfies the following condition, known as the valuated exchange axiom for all $X, Y \in \mathcal{P}_{d}$ :

For all $x \in X \backslash Y$, there exists some $y \in Y \backslash X$ such that

$$
v(X) \odot v(Y) \geq v(X \backslash\{x\} \cup\{y\}) \odot v(Y \backslash\{y\} \cup\{x\}) .
$$

Two valuated matroids $\mathcal{M}_{u}$ and $\mathcal{M}_{v}$ are called proportional if they are scalar multiples of one another.

The underlying matroid is the matroid on $S$ with bases given by

$$
\{X \subseteq S \mid v(X) \neq \infty\}
$$

and is denoted by $\mathcal{M}_{v}$.

Example 3.9.8. Let $\mathrm{S}$ be the set $\left\{x^{2}, x y, y^{2}\right\}$ of degree two monomials in the variables $x, y$, which we will write as $\left\{\left(\begin{array}{l}2 \\ 0\end{array}\right),\left(\begin{array}{l}1 \\ 1\end{array}\right),\left(\begin{array}{l}0 \\ 2\end{array}\right)\right\}$. Let $I$ be the ideal $\langle x+t y\rangle \subset \mathbb{C}\{\{t\}\}[x, y]$, and let $I_{2}$ be the degree-2 part of $I . I_{2}$ is generated by the binomials $b_{1}=x^{2}+t x y$ and $b_{2}=x y+t y^{2}$, and we also have $b_{3}=b_{1}-t b_{2}=x^{2}-t^{2} y^{2} \in I_{2}$. Valuating $b_{1}, b_{2}$, and $b_{3}$ respectively gives us the vectors $(0,1, \infty),(\infty, 0,1)$, and $(0, \infty, 2)$.

We show that these satisfy the valuated exchange axiom. Using the notation from Definition 3.9.7, put $X=b_{1}$ and $Y=b_{2}$. We have $x^{2} \in X \backslash Y$ and $t y^{2} \in Y \backslash X$, and

$$
v\left(x^{2}+t x y\right)+v\left(x y+t y^{2}\right)=(0,1, \infty)+(\infty, 0,1)=(\infty, 1, \infty) .
$$

We also have

$v\left(b_{1} \backslash\left\{x^{2}\right\} \cup\left\{t y^{2}\right\}\right)+v\left(b_{2} \backslash\left\{t y^{2}\right\} \cup\left\{x^{2}\right\}\right)=(\infty, 0,1)+(0,1, \infty)=(\infty, 1, \infty)$, so that the axiom is satisfied (since these are really the only choices of $X$ and $Y$ ).

The underlying matroid here is the matroid on the set $\left\{\left(\begin{array}{l}2 \\ 0\end{array}\right),\left(\begin{array}{l}1 \\ 1\end{array}\right),\left(\begin{array}{l}0 \\ 2\end{array}\right)\right\}$ with bases $\left\{\left(\begin{array}{l}2 \\ 0\end{array}\right),\left(\begin{array}{l}0 \\ 2\end{array}\right)\right\},\left\{\left(\begin{array}{l}2 \\ 0\end{array}\right),\left(\begin{array}{l}1 \\ 1\end{array}\right)\right\}$, and $\left\{\left(\begin{array}{l}1 \\ 1\end{array}\right),\left(\begin{array}{l}0 \\ 2\end{array}\right)\right\}$, i.e, the uniform matroid of rank 2 .

If we now consider the ideal $J=\left\langle t^{2} x+t^{3} y\right\rangle \subset \mathbb{C}\{\{t\}\}[x, y]$, and let $J_{2}$ be the degree-2 part of $J$, valuating the binomials in $J_{2}$ gives us the vectors $(2,3, \infty),(\infty, 2,3)$, and $(2, \infty, 4)$, which also give the bases for a valuated matroid. Since these bases are $2 \odot b_{i}$ for $i=1,2,3$, we see that the valuated matroid obtained from $J_{2}$ is proportional to that obtained from $I_{2}$.

Just like an ordinary matroid, a valuated matroid can also be defined in terms of its circuits. We will however postpone this definition until the end of Section 3.10 below. 
3.10. The Plücker Embedding and Tropical Linear Spaces. The Grassmannian, which we will define below, is a way of parametrising the $k$-dimensional linear subspaces of a vector space $\mathbb{K}^{n}$ overa field $\mathbb{K}$. This will be useful for us when it comes to studying the linear spaces of ideals and their tropicalisations. The Plücker embedding allows us to embed the Grassmannian into projective space. We will show in this section that for a linear space $L \subset \mathbb{K}^{n}$, the circuits of $\operatorname{Trop}(L)$ are determined by the valuations of the Plücker coordinates of $L$. We can apply this to the linear space of an ideal $I$ and its corresponding tropical linear space $\operatorname{Trop}(I)$. See Definition 3.10.12 below for exactly what is meant by a tropical linear space.

Most of the definitions in this section can be found in Chapter 6 of Algebraic Geometry by Joe Harris [10], and Chapters 2 and 4 of [17].

Definition 3.10.1. Let $\mathbb{K}$ be a field. Denote by $G(k, n)$ the set of $k$-dimensional linear subspaces of the vector space $\mathbb{K}^{n}$ over $\mathbb{K}$. For an abstract vector space $V$ we can also write $G(k, V)$. Since a $k$-dimensional subspace of $\mathbb{K}^{n}$ is the same as a $(k-1)$-plane in projective $(n-1)$ space, we can think of $G(k, n)$ as the subset of projective $(n-1)$-space consisting of all such $(k-1)$-planes and denote it by $\mathbb{G}(k, n)$.

We define the exterior algebra $\wedge^{k}(V)$ of a vector space $V$ to be the quotient of the tensor algebra $\otimes^{k} V$ by the ideal generated by all elements of the form $v \otimes w+w \otimes v$ for $v, w \in V$.

Let $V \cong \mathbb{K}^{n}$ be a vector space over $\mathbb{K}$. Let $W \subset V$ be the $k$ dimensional linear subspace spanned by the vectors $v_{1}, \ldots, v_{k}$. We can associate to $W$ the multivector

$$
\lambda=v_{1} \wedge \ldots \wedge v_{k} \in \wedge^{k}(V),
$$

which is determined up to scalars by $W$. This gives us a (well-defined) map of sets

$$
\phi: G(k, V) \rightarrow \mathbb{P}\left(\wedge^{k}(V)\right),
$$

where $\mathbb{P}\left(\wedge^{k}(V)\right)$ is projective space over $\wedge^{k}(V)$. This map is known as the Plücker embedding, and the homogeneous coordinates on $\mathbb{P}\left(\wedge^{k}(V)\right.$ are called Plücker coordinates on $G(k, V)$.

Choosing an identification $V \cong \mathbb{K}^{n}$ allows us to represent the plane $W$ by the $k \times n$ matrix $M_{W}$ whose rows are the vectors $v_{i}$, i.e., the subspace $W$ is the row space of the vectors $v_{i}$. The Plücker coordinates are the maximal minors of this matrix, which form a vector of length $\left(\begin{array}{l}n \\ k\end{array}\right)$ called the Plücker vector.

Example 3.10.2. We note here that it is possible for two matrices to have the same row space. Take for example the matrices

$$
A=\left[\begin{array}{llll}
1 & 2 & 3 & 4 \\
0 & 1 & 4 & 0
\end{array}\right], \quad B=\left[\begin{array}{cccc}
2 & 3 & 2 & 8 \\
-2 & -2 & 2 & -8
\end{array}\right] .
$$

$A$ and $B$ have the same row space $W$. When we calculate their Plücker vectors we get $(1,4,0,5,-4,-16)$ and $(2,8,0,10,-8,-32)$ respectively. 
Now, in $\mathbb{P}^{5}$, we have

$$
[1: 4: 0: 5:-4:-16] \sim[2: 8: 10: 0:-8:-32],
$$

so we see that the Plücker vector removes the ambiguity on how to represent $W$.

This is because if $A$ and $B$ are two matrices with the same row space, then $A=G B$ for some element $G \in \mathrm{GL}(k, \mathbb{K})$, and if $m$ is a minor of $A$ then the corresponding minor of $B$ is given by $|G| m$.

Definition 3.10.3. We will now define the Plücker relations and the Plücker ideal. Suppose $M_{W}$ is a $k \times n$ matrix whose row space represents the plane $W$. Let the columns of $M_{W}$ be indexed by te set $[n]=$ $\{1, \ldots, n\}$, let $J$ be a subset of $[n]$, and let $|J|=k$. Denote the coordinate ring of $\mathbb{P}\left(\begin{array}{l}n \\ k\end{array}\right)-1$ by

$$
\mathbb{K}[\mathbf{p}]=\mathbb{K}\left[p_{J}: J \subset[n],|J|=k\right],
$$

where $p_{J}$ is the $k \times k$ minor of $M_{W}$ indexed by $J$.

The Plücker ideal $I_{k, n}$ is the set of all polynomials in $\mathbb{K}[\mathbf{p}]$ that vanish on all vectors of $k \times k$ minors for all $k \times n$ matrices. This is a homogeneous prime ideal of all polynomial relations among $k \times k$ minors and is generated by the Plücker relations, which we define below.

Fix subsets $J, L \subset[n]$ with $|J|=k-1$ and $|L|=k+1$. For $l \in L$, define the $\operatorname{sign} \operatorname{sgn}(l ; J, L)$ to be $(-1)^{m}$ where $m$ is the number of elements $l^{\prime} \in L$ with $l<l^{\prime}$ plus the number of elements $j \in J$ with $j>l$. The Plücker relation $\mathcal{P}_{J, L}$ is the following homogeneous quadric

$$
\mathcal{P}_{J, L}=\sum_{l \in L} \operatorname{sgn}(l ; J, L) \cdot p_{J \cup l} \cdot p_{L \backslash l},
$$

where $p_{J \cup l}=0$ if $l \in J$.

Example 3.10.4. If we take the matrix $A$ from the previous example so that

$$
A=\left[\begin{array}{llll}
1 & 2 & 3 & 4 \\
0 & 1 & 4 & 0
\end{array}\right]
$$

and label the columns from left to right by the elements in the set [4], then fix $J=\{1\}$ and $L=\{2,3,4\}$, we get $\operatorname{sgn}(2)=1, \operatorname{sgn}(3)=-1$, and $\operatorname{sgn}(4)=1$. Then

$$
\mathcal{P}_{J, L}=p_{12} p_{34}-p_{13} p_{24}+p_{14} p_{23} .
$$

Substituting the Plücker coordinates into this gives $1 \cdot(-16)-4 \cdot(-4)+$ $0 \cdot 5=0$, as expected.

Example 3.10.5. Now take the matrix

$$
A^{\prime}=\left[\begin{array}{lll}
1 & 2 & 3 \\
0 & 1 & 4
\end{array}\right]
$$


with columns indexed by the set [3] and fix $J=\{1\}$ and $L=\{1,2,3\}$. We get $\operatorname{sgn}(1)=1, \operatorname{sgn}(2)=-1$, and $\operatorname{sgn}(3)=1$. We then get

$$
\mathcal{P}_{J, L}=p_{11} \cdot p_{23}-p_{12} \cdot p_{13}+p_{13} \cdot p_{12}=0 .
$$

We get similar results for the other possible choices for $J$ and $L$. In this case there are no non-trivial Plücker relations.

Below is the simplest example of a nontrivial Grassmannian, the case that Plücker first looked at. The Plücker embedding is a generalisation of this construction.

Example 3.10.6. We will consider lines in real projective 3-space, $\mathbb{P}_{\mathbb{R}}^{3}$. Lines in $\mathbb{P}_{\mathbb{R}}^{3}$ can be parametrised by four homogemeous coordinates $[x: y: z: t]$. The space of 2-dimensional subspaces of $\mathbb{P}_{\mathbb{R}}^{3}$ can thus be paramatrised by the two vectors $v_{1}=\left(x_{1}, y_{1}, z_{1}, t_{1}\right)$ and $v_{2}=\left(x_{2}, y_{2}, z_{2}, t_{2}\right)$. A plane $W$ can be represented by the matrix

$$
M_{W}=\left[\begin{array}{llll}
x_{1} & y_{1} & z_{1} & t_{1} \\
x_{2} & y_{2} & z_{2} & t_{2}
\end{array}\right]
$$

The Plücker coordinates are the maximal minors of this matrix: $x_{1} y_{2}-$ $x_{2} y_{1}, x_{1} z_{2}-x_{2} z_{1}, x_{1} t_{2}-x_{2} t_{1}, y_{1} z_{2}-y_{2} z_{1}, y_{1} t_{2}-y_{2} t_{1}$, and $z_{1} t_{2}-z_{2} t_{1}$.

If we index the columns of $M_{W}$ by the set [4] and take $J$ and $L$ as in Example 3.10 .4

We will now see how this relates to linear spaces and matroids.

Definition 3.10.7. Let $I$ be a homogeneous ideal in $\mathbb{K}\left[x_{0}, \ldots, x_{n}\right]$, and let the grading of $x_{i}=1$. Suppose $I$ is generated by a single homogeneous polynomial $p(x)$ of degree $d$. Then $I$ can be decomposed into the following sum:

$$
I=I_{d} \oplus I_{d+1} \oplus I_{d+2} \oplus \ldots
$$

We can represent each graded summand $I_{n}$ of $I$ as a matrix, by labelling the columns with monomials in $x_{0}, \ldots, x_{n}$ of degree $n$ and the rows with the products of $p(x)$ with every degree $n-d$ monomial in $x_{0}, \ldots, x_{n}$, then entering the coefficients of each monomial appearing in each row (all other entiries are zeros.) We call this the Macaulay matrix of $I$ in degree $n$.

The rows of the Macaulay matrix in each degree are our vectors $v_{i}$ from Definition 3.10.1; the Plücker coordinates, i.e., the maximal minors of the Macaulay matrices, give us homogeneous coordinates for their span.

Recall that the determinant of a matrix is zero if and only if the vectors that make up its rows or columns are linearly dependent; using this basic fact from linear algebra, we find that the non-zero Plücker coordinates correspond to the bases of a matroid, i.e., the maximal independent sets. 
Definition 3.10.8. Let $\mathbb{K}$ be a field and $\mathbb{T}$ be the tropical semiring. Let $v: \mathbb{K} \rightarrow \mathbb{T}$ be a valuation on $\mathbb{K}$. Suppose some linear space over $\mathbb{K}^{n}$ has the Plücker vector $\left\{p_{i}\right\}$. We define the tropical Plücker vector to be the component-wise valuation $\left\{v\left(p_{i}\right)\right\}$ of this vector. The tropical Plücker coordinates are the valuations of the classical Plücker coordinates.

We will show below in Proposition 3.10.10 how we can use the Plücker coordinates to find the (valuated) circuits of a (valuated) matroid.

Lemma 3.10.9. Let $\mathcal{M}=(\mathcal{E}, \mathcal{I})$ be a matroid of rank $d$ and let $D$ be a minimal dependent set. Then $D=I \cup\{j\}$, where $I$ is an independent set and $j \in D \backslash I$, and we have $D \subset B \cup\{j\}$, where $B$ is some basis of $\mathcal{M}$.

Proof. By definition, if we take any element $j \in D$, then $D \backslash\{j\}$ is independent or else $D$ is not minimal, thus $D=I \cup\{j\}$ for some independent set $I$. All independent sets are contained in some basis $B$ of $\mathcal{M}$ by the definition of a matroid. Thus $I \subset B$ for some basis $B$, which implies $I \cup\{j\} \subset B \cup\{j\}$.

Proposition 3.10.10. Let $\mathcal{M}$ be the underlying matroid of a valuated matroid of rank $d$ on the set $S=M_{k} n_{k}$ of degree $k$ monomials in the variables $\left[x_{1}, \ldots, x_{n}\right]$. Let $U \subset M_{k} n_{k}$ be any dependent set with $|U|=d+1$. Then the sum

$$
\sigma_{U}=\sum_{i \in U} p_{U \backslash\{i\}} \boldsymbol{x}_{i} \in \mathbb{T}\left[x_{1}, \ldots, x_{n}\right],
$$

where $\boldsymbol{x}_{i}$ is the monomial corresponding to the $i^{\text {th }}$ element of $U$, is a circuit of $\mathcal{M}$.

Proof. We can write the subset $U=B \cup\{j\}$ for some basis $B$ of $\mathcal{M}$, so that $U \cup\{i\}$ becomes $B \cup\{j\} \cup\{i\}$. Then we have

$$
\sigma_{U}=\sum_{i} P_{B \cup\{j\} \backslash\{i\}} \mathbf{x}_{i}
$$

The coefficient of $\mathbf{x}_{i}$ in $\sigma_{U}$ is non-vanishing if and only if $i$ belongs to a minimal dependent set in $U$ (since in that case removing $i$ leaves us with an independent set). Since there exists a unique minimal dependent set in any set with cardinality $d+1$, it follows that cycling through all possible subsets $U$ will give us a way of generating all of the circuits of $\mathcal{M}$.

Definition 3.10.11. The circuits that Proposition 3.10.10 gives are known as the fundamental circuits of the valuated matroid $\mathcal{M}_{v}$. Let $C$ be any fundamental circuit of $\mathcal{M}_{v}$. We define the valuated circuits of $\mathcal{M}_{v}$ to be any vector of the form $\lambda \odot C$, where $\lambda$ is a non-zero element of the tropical semiring $\mathbb{T}^{S}$. We will denote the set of valuated circuits by $\mathcal{C}\left(\mathcal{M}_{v}\right)$.

The set $\mathcal{C}\left(\mathcal{M}_{v}\right)$ generates a subsemimodule of $\mathbb{T}^{S}$, elements of which are called vectors of $\mathcal{M}_{v}$. We denote the set of vectors of $\mathcal{M}_{v}$ by $\mathcal{V}\left(\mathcal{M}_{v}\right)$. 
Definition 3.10.12 (Tropical Linear Space). If the set of vectors $\mathcal{V}\left(\mathcal{M}_{v}\right)$ of a valuated matroid $\mathcal{M}_{v}$ live in the tropical semiring $\mathbb{T}^{S}$, then we call $\mathcal{V}\left(\mathcal{M}_{v}\right)$ a tropical linear space.

Example 3.10.13. Following on from Example 3.9.8, we take the degree-2 piece $I_{2}$ of the homogeneous ideal $I=\langle x+t y\rangle \subset \mathbb{C}\{\{t\}\}[x, y]$. We will label each respective element of the set $\operatorname{Mon}_{2}=\left\{\left(\begin{array}{l}2 \\ 0\end{array}\right),\left(\begin{array}{l}1 \\ 1\end{array}\right),\left(\begin{array}{l}0 \\ 2\end{array}\right)\right\}$ by $\{1,2,3\}$ to avoid unreadable notation. From the Macaulay matrix in degree 2, we can calculate the Plücker coordinates as follows: $p_{12}=1$, $p_{13}=t$, and $p_{23}=t^{2}$. We take $U=\{1,2,3\}$ and we get

$$
\sigma_{U}=t^{2} x^{2}+t x y+y^{2} \text {. }
$$

This gives us the fundamental valuated circuit $(2,1,0)$ so that the set of vectors of the valuated matroid is simply the set

$$
\left\{\lambda \odot(2,1,0): \lambda \in \mathbb{T}^{3}\right\} .
$$

3.11. Tropical Ideals. In their paper Tropical Ideals [15] Diane Maclagan and Felipe Rincon introduce tropical ideals as a special class of ideals in the tropical polynomial semiring, and discuss some of their properties. Since the tropicalisations of classical ideals are strictly included in this class, and tropical ideals have some nice properties that mirror their classical counterparts, it is important that we understand their structure. One invariant that the authors consider is the Hilbert function. They show that given a classical ideal $I$, then the Hilbert function of $J=\operatorname{trop}(I)$ agrees with the Hilbert function of $I$, and thus Hilbert functions are preserved under tropicalisation.

Two of the main results given in the paper are as follows:

Theorem 3.11.1 (Theorem 1.3 [15]). There is no infinite ascending chain $I_{1} \subset I_{2} \subset I_{3} \subset \ldots$ of tropical ideals in $\mathbb{T}\left[x_{1}, \ldots, x_{n}\right]$.

Theorem 3.11.2 (Theorem 1.4/Corollary 5.17 [15]). If $I \subseteq \mathbb{T}\left[x_{1}, \ldots, x_{n}\right]$ is a tropical ideal, then the variety $V(I) \subseteq \mathbb{T}^{n}$ is empty if and only if $I$ is the unit ideal $\langle 0\rangle$.

If $I$ is a homogeneous tropical ideal in $\mathbb{T}\left[x_{0}, \ldots, x_{n}\right]$ then the variety $V(I) \subseteq \operatorname{trop}\left(\mathbb{P}^{n}\right)$ is empty if and only if there exists $d>0$ such that $\left\langle x_{0}, \ldots, x_{n}\right\rangle^{d} \subseteq I$.

Definition 3.11.3. The paper begins by defining a tropical ideal as follows. If we denote the tropical semiring $(\mathbb{R} \cup\{\infty\}, \oplus, \odot)=(\mathbb{R} \cup$ $\{\infty\}$, min, +$)$ by $\mathbb{T}$ and let $I \subset \mathbb{T}\left[x_{1}, \ldots, x_{n}\right]$ be an ideal, then we say that $I$ is a tropical ideal if for each degree $d \geq 0$ the set of polynomials $I_{\leq d}$ of degree at most $d$ is a tropical linear space (i.e., the set of vectors of a valuated matroid).

The authors give the following "monomial elimination axiom" (equivalent to the circuit elimination axiom):

For any $f=\sum f_{\mathbf{u}} \mathbf{x}^{\mathbf{u}}, g=\sum g_{\mathbf{u}} \mathbf{x}^{\mathbf{u}} \in I_{\leq d}$ and any monomial $\mathbf{x}^{\mathbf{u}}$ for which $f_{\mathbf{u}}=g_{\mathbf{u}} \neq \infty$, there exists $h=\sum h_{\mathbf{u}} \mathbf{x}^{\mathbf{u}} \in I_{\leq d}$ such that $h_{\mathbf{u}}=\infty$ 
and $h_{\mathbf{v}} \geq \min \left(f_{\mathbf{v}}, g_{\mathbf{v}}\right)$ for all monomials $\mathbf{x}^{\mathbf{v}}$, with equality whenever $f_{\mathbf{v}} \neq g_{\mathbf{v}}$.

Example 3.11.4. Let $I=\operatorname{trop}(\langle x+t y\rangle) \in \mathbb{T}[x, y]$ and let $f=x^{2} \oplus$ $1 \odot x y, g=1 \odot x y \oplus 2 \odot y^{2}$ and $h=x^{2} \oplus 2 \odot y^{2}$, where $f, g, h \in I_{2}$.

Definition 3.11.5. Now let $I \subset \mathbb{T}\left[x_{0}, \ldots, x_{n}\right]$ be a homogeneous ideal, and let $\mathrm{Mon}_{d}$ be the set of monomials of degree $d$ in the variables $x_{i}$. We say that $I$ is a homogeneous tropical ideal if for each degree $d \geq 0$, the degree $d$ part $I_{d}$ of $I$ is the collection of vectors of a valuated matroid $\mathcal{M}_{d}$ on $\operatorname{Mon}_{d}$.

Definition 3.11.6. Let $\mathcal{S}=\left(\mathcal{M}_{d}\right)_{d \geq 0}$ be a sequence of valuated matroids with values in $\mathbb{T}$, where the ground set for $\mathcal{M}_{d}$ is the set of monomials $\mathrm{Mon}_{d}$ of degree $d$. We say that $\mathcal{S}$ is a compatible sequence if the $\mathbb{T}$-subsemimodule generated by the set of vectors $\left\{\mathcal{V}\left(\mathcal{M}_{d}\right): d \geq 0\right\}$ is a homogeneous ideal. In terms of circuits, that is for any circuit $C \in \mathcal{C}\left(\mathcal{M}_{d}\right)$, we have that $x_{i} \odot C$ is a sum of circuits of $\mathcal{M}_{d+1}$.

3.12. Tropical Elliptic Curves and the $j$-invariant. In this section we will summarise some of the relevant information in Katz, Markwig and Markwig's papers [12] and [13] dealing with tropical elliptic curves and $j$-invariants. Their research shows that the negative of the cycle length of a tropical elliptic curve is an appropriate tropical analogue of the $j$-invariant, and that this equals the generic valuation of the $j$-invariant. The main result from [12] is as follows (definitions will be given below):

Theorem 3.12.1 (Theorem 4.1, [12]). Let $\mathcal{C}$ be a plane cubic curve whose tropicalisation trop $(\mathcal{C})$ is given by the tropical polynomial

$$
\min \left\{u_{i j}+i x+j y\right\}
$$

where $i+j \leq 3$ and assume that $\operatorname{trop}(\mathcal{C})$ has a cycle.

Then the negative of the generic valuation at $\boldsymbol{u}=\left(u_{i j}\right)_{i+j \leq 3}$ is equal to the cycle length $\operatorname{cl}(\boldsymbol{u})$ of $\operatorname{trop}(\mathcal{C})$, i.e.,

$$
-v_{\boldsymbol{u}}(j)=\operatorname{cl}(\boldsymbol{u})
$$

Furthermore, if the marked subdivision dual to $\operatorname{trop}(\mathcal{C})$ is a triangulation, then $\boldsymbol{v}_{\boldsymbol{u}}(j)=v(j(f))$ where $f=\sum_{i+j \leq 3} a_{i j} x^{i} y^{j}$ is any elliptic curve with coefficients $a_{i j}$ satisfying $v\left(a_{i j}\right)=u_{i j}$.

In [12] the authors focus on tropical plane cubics, that is curves defined by an equation of the form $f=\sum_{i+j \leq 3} a_{i j} x^{i} y^{j}$.

Definition 3.12.2. We say that a plane tropical cubic has a cycle if the point $(1,1)$ is a vertex of a marked polytope in the dual marked subdivision. The cycle is defined to be the union of the bounded edges of the curve that are dual to the facets of the marked subdivision which emanate from the point $(1,1)$. 
Let $E$ be a bounded edge of a plane tropical curve, with direction vector $v(E)$. We define the lattice length of $E$ to be

$$
\frac{\|E\|}{\|v(E)\|}
$$

where $\|\cdot\|$ is the Euclidean norm. We can then define the cycle length of a plane tropical cubic to be the sum of the lattice lengths of the bounded edges that form the cycle.

If a plane tropical cubic does not have a cycle, but the point $(1,1)$ is contained within an edge of one of the polytopes in the corresponding marked subdivision, we can define the generalised cycle length to be the lattice length of the edge of the tropical curve dual to the edge containing the point $(1,1)$ multiplied by 4 .

Example 3.12.3. Let $\omega^{2}=2$ in some field of characteristic 3 and let $\mathcal{E}$ be the elliptic curve defined by the ideal

$$
\left\langle f_{\mathcal{E}}\right\rangle=\left\langle y^{2}+\omega t^{-1} x y-x^{3}-(1+\omega) t^{2} x-t^{2}\right\rangle \subset \overline{\mathbb{F}_{3}}\{\{t\}\}[x, y] .
$$

Tropicalising this gives us

$$
\operatorname{trop}\left(f_{\mathcal{E}}\right)=\min \{2 y,-1+x+y, 3 x, 2+x, 2\}
$$

which corresponds to the following marked subdivision:

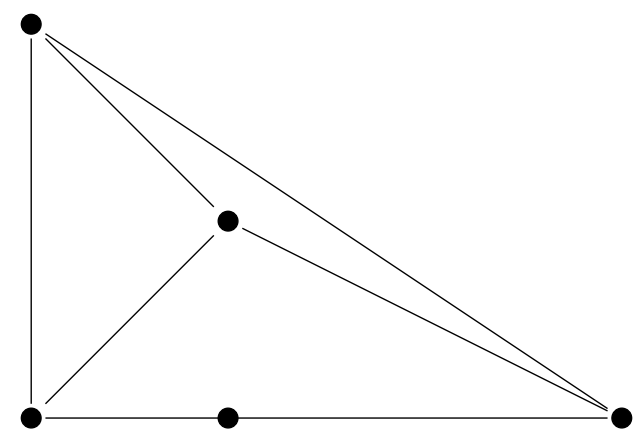

Since the point $(1,1)$ is visible as a vertex in the marked subdivision, we know that $\mathcal{E}$ has a cycle. Some quick calculations yield the dual tropical hypersurface below. 


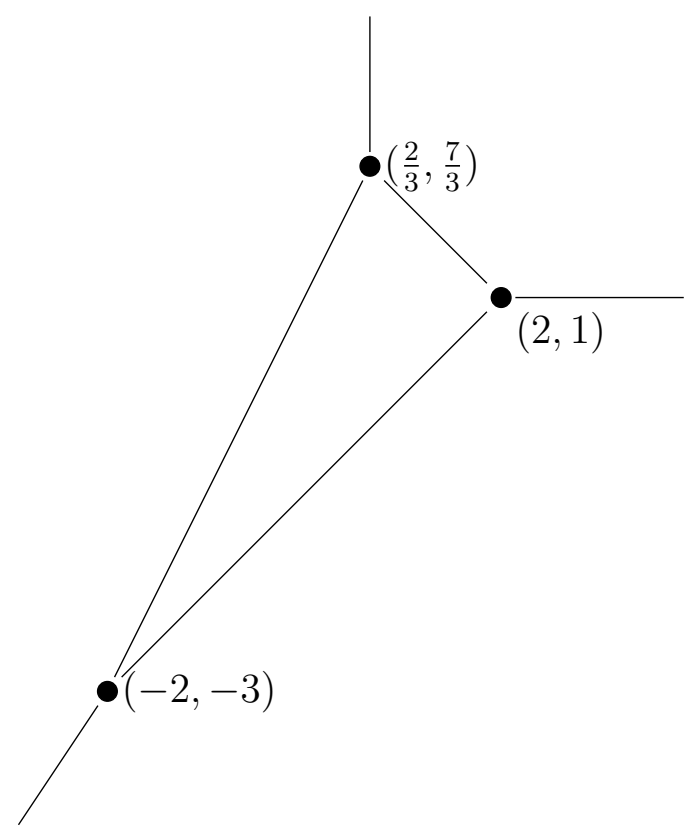

The vertices of the cycle are the points $(-2,-3),(2,1)$ and $\left(\frac{2}{3}, \frac{7}{3}\right)$. From this we can work out the cycle length as

$$
\frac{4 \sqrt{2}}{\sqrt{2}}+\frac{8 \sqrt{5}}{3 \sqrt{5}}+\frac{4 \sqrt{2}}{3 \sqrt{2}}=8
$$

Thus the tropical $j$-invariant is -8 .

Now we consider the following change of variables, putting $y^{\prime}=$ $y+\frac{\omega}{2} t^{-1} x$ :

$$
\begin{aligned}
y^{2}+\omega t^{-1} x y & =x^{3}+(1+\omega) t^{2} x+t^{2} \\
\left(y+\frac{\omega}{2} t^{-1} x\right)^{2}-\left(\frac{\omega}{2} t^{-1} x\right)^{2} & =x^{3}+(1+\omega) t^{2} x+t^{2} \\
y^{\prime 2} & =x^{3}+2 t^{-2} x^{2}+(1+\omega) t^{2} x+t^{2} .
\end{aligned}
$$

Denote $y^{\prime 2}-x^{3}-2 t^{-2} x^{2}-(1+\omega) t^{2} x-t^{2}$ by $f_{\mathcal{E}}^{\prime}$, and denote the elliptic curve it defines by $\mathcal{E}^{\prime}$. We have

$$
\operatorname{trop}\left(f_{\mathcal{E}}^{\prime}\right)=\min \{2 y, 3 x,-2+2 x, 2+x, 2\}
$$

which results in the following marked subdivision of the Newton polytope: 


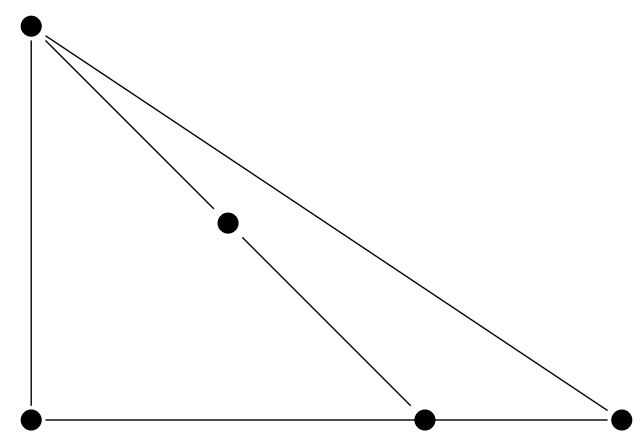

Since $(1,1)$ is not a vertex in the marked subdivision, $\mathcal{E}^{\prime}$ does not have a cycle. However, we can calculate the generalised cycle length. The tropical hypersurface $\operatorname{trop}\left(f_{\mathcal{E}}^{\prime}\right)$ has vertices $(-2,-3)$ and $(2,1)$. The lattice length is

$$
\frac{4 \sqrt{2}}{2 \sqrt{2}}=2
$$

and therefore the generalised cycle length is $4 \times 2=8$. Thus the tropical $j$-invariant of $\mathcal{E}^{\prime}$ is also -8 .

We can calculate the classical $j$-invariant of $\mathcal{E}^{\prime}$ as follows, using the forumula for fields of characteristic 3 :

$$
\begin{aligned}
j\left(\mathcal{E}^{\prime}\right) & =\frac{B^{6}}{B^{2} C^{2}-C^{3}-B^{3} D} \\
& =\frac{\left(2 t^{-2}\right)^{6}}{\left(2 t^{-2}\right)^{2}\left((1+\omega) t^{2}\right)^{2}-\left((1+\omega) t^{2}\right)^{3}-\left(2 t^{-2}\right)^{3} t^{2}} \\
& =\frac{t^{-12}}{t^{-4}+2 \omega+(2+\omega) t^{6}} .
\end{aligned}
$$

Valuating, we get $v\left(j\left(\mathcal{E}^{\prime}\right)\right)=-12-(-4)=-8$, which is what we would expect.

In Example 5.7.1 later on, we will see that sometimes the generic valuation and the valuation of the $j$-invariant of a curve don't match; in this case we ask, given just the data of the tropical ideal, is there a way of finding out the true valuation of the $j$-invariant?

\section{Quadratics and Generalisations}

The structure of the tropical ideal $\operatorname{trop}(I)$ contains more information than the tropical variety $\operatorname{trop}(\mathcal{V}(I))$, and we want to know how many of the classical invariants of a scheme we can get from the tropical ideal structure. In particular the question we would like to answer is whether in the case of tropical elliptic curves, the tropical ideal tells us anything about the valuation of the $j$-invariant in those cases where it does not match the generic valuation. Since the discriminant of a cubic appears in the formula for the $j$-invariant, we begin by investigating whether the tropical ideal $\operatorname{trop}(\langle p(x, z)\rangle)$ determines the valuation of the discriminant of the homogeneous polynomial $p(x)$. 
In the case of the quadratic polynomial $A x^{2}+B x z+C z^{2}$ it was shown in a paper by Fink, Giansiracusa and Giansiracusa [9] that over fields of characteristic 0 the valuation of the discriminant $\Delta_{2}=B^{2}-4 A C$ is not determined by the valuations of the Plücker coordinates of the ideal $\left\langle A x^{2}+B x z+C z^{2}\right\rangle$. In this section we will give a brief account of this research, then we will see that the same holds for biquadratics of the form $x^{4}+B x^{2} z^{2}+C z^{4}$, and multiquadratics of the form $x^{2 n}+B x^{n} z^{n}+C z^{2 n}$.

4.1. The Quadratic Case. It is well understood due to [9] what happens in the case of a quadratic of the form $A x^{2}+B x z+C z^{2}$. Here we will go through what is already known to lay the foundations for the generalisation of this material in the sections that follow. We will begin by proving Proposition 4.1.1, then we can use a change of variables to show that the Plücker coordinates never share a root with the discriminant, and therefore the tropicalisation does not determine the vauation of the discriminant.

Proposition 4.1.1. The Plücker coordinates $\beta_{n}$ generated in each degree $d$ (where $d=2+n$ ) by taking the minor where each entry in the leading diagonal is $B$ are determined by the recurrence relation

$$
\beta_{i+1}=B \beta_{i}-A C \beta_{i-1},
$$

and initial condition $\beta_{-1}=1, \beta_{-2}=0$; furthermore that all Plücker coordinates are determined by this relation.

Proof. Below are the Macaulay matrices for degrees $n=2+d$, where $d=0,1,2,3$. We will generate the $\beta_{i}$ using the minor obtained by taking the central square submatrix, dropping the first and last column, for which every entry in the diagonal is $B$.

$$
\begin{aligned}
& \beta_{0}=B \\
& \beta_{1}=B^{2}-A C \\
& \beta_{2}=B^{3}-2 A C B \\
& \beta_{3}=B^{4}-3 A C B+(A C)^{2}\left[\begin{array}{cccccc}
A & B & C & 0 & 0 & 0 \\
0 & A & B & C & 0 & 0 \\
0 & 0 & A & B & C & 0 \\
0 & 0 & 0 & A & B & C
\end{array}\right]
\end{aligned}
$$

Calculating the minor obtained from taking $B$ as the central diagonal, we obtain the recurrence relation as follows: expand down the far left column, to get $\beta_{i+1}=B \beta_{i}-A \ldots$, then expand along the top row to get $\beta_{i+1}=B \beta_{i}-A C \beta_{i-1}$ to obtain the result. 
Now we must consider whether this recurrence relation determines all Plücker coordinates, which can be summarised by the following lemma.

Lemma 4.1.2. The valuations of all Plücker coordinates are determined by the valuations $v(A), v(B)$, and $V(C)$ of $A, B$, and $C$ respectively, and the valuations $v\left(\beta_{i}\right)$ of $\beta_{i}$ for all $i$.

Proof. We will show inductively that this is true. Assume that in degree $k$ and below, all Plücker coordinates are determined by the $(k-2) \times(k-2)$ minor

$$
\left[\begin{array}{ccccc}
B & C & 0 & \ldots & 0 \\
A & B & C & \ldots & 0 \\
\vdots & & \ddots & & \vdots \\
0 & & \ldots & & B
\end{array}\right]
$$

and consider the following $(k-1) \times(k-2)$ submatrix of the Macaulay matrix in degree $k+1$ :

$$
\left[\begin{array}{ccccc}
C & 0 & 0 & \ldots & 0 \\
B & C & 0 & \ldots & 0 \\
A & B & C & \ldots & 0 \\
\vdots & & \ddots & & \vdots \\
0 & & \ldots & & B
\end{array}\right]
$$

We have a choice of three columns we can add to this in order to produce a square submatrix of the Macaulay matrix in degree $k+1$. Either we choose the column with zero in every entry except for the top entry which is $A$, the column with zero for every entry except for the bottom entry which is $C$, or the column with $B$ for the top entry, $C$ for the next entry, and zeroes everywhere else. In the first and second case, we simply obtain a multiple of the maximal Plücker coordinate for degree $k$, and in the third case we obtain the minor with central diagonal consisting only of $B$.

A similar argument can be made for replacing any column in the minor with central diagonal $B$ with the column of either the first or second case above (or both). Thus the lemma is proved, completing the proof of Proposition 4.1.1.

Given the ideal $I=\left\langle A x^{2}+B x z+C z^{2}\right\rangle$, the tropical ideal $\operatorname{Trop}(I)$ determines and is determined by $v(A), v(B), v(C)$, and the sequence of numbers $v\left(\beta_{i}\right)$. Since $v(A), v(B)$, and $v(C)$ determine the generic valuations of each $\beta_{i}$, we can compare each $v\left(\beta_{i}\right)$ with the corresponding generic valuation and ask what information we can get when these values don’t agree. 
We then obtain the following sequence of Plücker coordinates $\beta_{i}$ in degree $d$, where $d=2+i$ :

$$
\begin{aligned}
& \beta_{0}=B \\
& \beta_{1}=B^{2}-A C \\
& \beta_{2}=B^{3}-2 A C B \\
& \beta_{3}=B^{4}-3 A C B^{2}+(A C)^{2} \\
& \beta_{4}=B^{5}-4 A C B^{3}+3(A C)^{2} B \\
& \beta_{5}=B^{6}-5 A C B^{4}+6(A C)^{2} B^{2}-(A C)^{3}
\end{aligned}
$$

Now, take the polynomial $A x^{2}+B x z+C z^{2}$ and scale the coefficients so that $B=1$, then write $u=A C$. The $\beta_{i}$ then become:

$$
\begin{aligned}
& \beta_{0}=1 \\
& \beta_{1}=1-u \\
& \beta_{2}=1-2 u \\
& \beta_{3}=1-3 u+u^{2} \\
& \beta_{4}=1-4 u+3 u^{2} \\
& \beta_{6}=1-5 u+6 u^{2}-u^{3} \\
& \beta_{7}=1-6 u+10 u^{2}-4 u^{3}
\end{aligned}
$$

The coefficients of each power of $u$ are the $n$-simplex numbers (where the $k^{\text {th }} n$-simplex number is given by the binomial coefficient $\left.\left(\begin{array}{c}k+(n-1) \\ n\end{array}\right)\right)$; this can be used to write a general formula for $\beta_{i}$.

The next step is to consider the Plücker coordinates in terms of the roots of the quadratic. Suppose we have the polynomial $\hat{A} x^{2}+x z+\hat{C} z^{2}$, (already scaled so that $B=1$ ). We can write it in the form

$$
x^{2}+\frac{1}{\hat{A}} x z+\frac{\hat{C}}{\hat{A}}=(x-p)(x-q)
$$

for some $p, q$ which are roots of the polynomial. We can rearrange this to get

$$
\hat{A} \hat{C}=\frac{p q}{(p+q)^{2}} .
$$

We now consider the polynomials given by $(p+q)^{i+1} \beta_{i}$ :

$$
\begin{aligned}
(p+q) \beta_{0} & =p+q \\
(p+q)^{2} \beta_{1} & =p^{2}+p q+q^{2} \\
(p+q)^{3} \beta_{2} & =p^{3}+p^{2} q+p q^{2}+q^{3}
\end{aligned}
$$


These appear to be the elementary symmetric polynomials in the variables $p, q$. To prove this inductively for all $i$, consider the recurrence relation obtained from (1)

$$
(p+q)^{i+2} \beta_{i+1}=(p+q)(p+q)^{i+1} \beta_{i}-p q(p+q)^{i} \beta_{i-1} .
$$

If we assume that $\beta_{k}$ and $\beta_{k-1}$ are the elementary symmetric polynomials of degree $k+1$ and $k$ (respectively) in $p, q$, then thefirst term in the above relation gives all the monomials in degree $k+2$ divisible by $p$ plus all the monomials divisible by $q$, with the monomials divisible by $p q$ being counted twice. The second term subtracts one copy of each monomial divisble by $p q$, so that we again get the degree $k+2$ elementary symmetric polynomial in $p, q$.

Since these polynomials are homogeneous in two variables, they live in projective space and depend on the ratio $[p: q]$, which is the same as the ratio $\left[\frac{p}{q}: 1\right]$. Put $v=\frac{p}{q}$ and divide $(p+q)^{i+1} \beta_{i}$ by $q^{i+1}$, then after this change of variables we get that the $\beta_{i}$ are equal to $1+v+\ldots+v^{i}$. We have

$$
(1-v)\left(1+v+\ldots+v^{i}\right)=v^{i+1}-1,
$$

so that the roots of the $\beta_{i}$ are simply the nontrivial $(i+1)^{t h}$ roots of unity.

Now, in terms of the roots $p, q$, the discriminant of our quadratic is given by

$$
1^{2}-4 \hat{A} \hat{C}=1-\frac{4 p q}{(p+q)^{2}} .
$$

Multiplying through by $\frac{(p+q)^{2}}{q^{2}}$ gives

$$
\frac{(p+q)^{2}}{q^{2}}-\frac{4 p}{q}
$$

which, in terms of $v$, gives

$$
(v+1)^{2}-4 v=(v-1)^{2} .
$$

Thus the roots of the discriminant in terms of $v$ are both equal to 1 and can never coincide with the roots of the Plücker coordinates. This means that the valuation of any Plücker coordinate and of the discriminant cannot both deviate from the generic valuation. Thus if we find that one does, then we know that the other definitely does not.

Alternatively, we can scale the coefficients so that $A=1$. This is the convention we will adopt for most of the work that follows. The proof given above still works, but instead we find that, in terms of the roots $p, q, \beta_{k}$ is just the elementary symmetric polynomial in $p$ and $q$ of degree $k+1$, multiplied by $(-1)^{k+1}$. Again, this means that we can write $t=\frac{p}{q}$, and we find that the roots of the $\beta_{k}$ are the nontrivial $(k+1)^{t h}$ roots of unity, while discriminant has a single repeated root at 1 . 
Example 4.1.3. Here is an example of a quadratic polynomial where the valuation of the discriminant deviates from the generic valuation. Take the quadratic

$$
q(x, z)=x^{2}-\left(2 t^{2}+2 t^{3}+t^{4}\right) x z+\left(t^{4}+2 t^{5}+t^{6}\right) z^{2},
$$

which has the two distinct roots, $t^{2}$ and $\left(t+t^{2}\right)^{2}$ (both with valuation 2). We can calculate its discriminant

$$
\begin{aligned}
B^{2}-4 A C & =\left(2 t^{2}+2 t^{3}+t^{4}\right)^{2}-4\left(t^{4}+2 t^{5}+t^{6}\right) \\
& =4 t^{6}+4 t^{7}+t^{8} .
\end{aligned}
$$

The discriminant is clearly non-zero, and has valuation 6 . However, the generic valuation of $\Delta$ is

$$
\min \{2 v(B), v(C)\}=\min \{4,4\}=4 ;
$$

note that since the minimum is achieved twice, the discriminant vanishes tropically, implying that $\operatorname{trop}(q)$ has a repeated root. Indeed,

$$
\operatorname{trop}(q)=\min \{2 x, 2+x+z, 4+2 z\}
$$

has the root $z=x-2$ with multiplicity 2 .

We always lose information when passing to the tropical world in this case, we can no longer tell from the valuations of the roots, coefficients, or the generic valuation of the discriminant that $q(x, z)$ has two distinct roots. A strong motivation, therefore, for studying tropical ideals is to know whether such information can be recovered from the tropical ideal structure.

4.1.1. The Quadratic Case in Charactistic p. Building on the work outlined in the previous section, we investigate what happens over field of characteristic $p$ and find that the situation is somewhat different. For small values of $p$, the valuation of the discriminant often is determined by the valuations of the Plücker coordinates over the field $\mathbb{F}_{p}$. Characteristic 2 is a special case, since the usual discriminant $B^{2}-4 A C$ does not apply, since the second term vanishes. While there are analogues for the discriminant in fields of characteristic 2 (see for example [1]), we will not explore them here. Therefore we will restrict our attention to $p=3,5,7,11$. We will scale the quadratic $A x^{2}+B x z+C z^{2}$ such that $B=1$, and write $A C=u$. Thus the discriminant becomes $1-4 u$.

- When $p=3$, we have $\Delta=1+2 u=2(u+2)$, and $\beta_{11}=$ $2(u+2)(u+1)^{3}=\Delta(u+1)^{3}$.

- When $p=5$, we have $\Delta=u+1$, and $\beta_{3}=(u+1)^{2}=\Delta^{2}$.

- When $p=7$, we have $\Delta=1+3 u=3(u+5)$, and $\beta_{6}=6(u+5)^{3}=$ $\Delta^{3}$.

- When $p=11$, we have $\Delta=1+7 u=7(u+8)$, and $\beta_{10}=$ $10(u+8)^{5}=\Delta^{5}$.

This will also be true for any extension of the field $\mathbb{F}_{p}$. Thus for any field of characteristic $p=3,5,7,11$, the discriminant appears as 
a factor in the Plücker coordinates of $\left\langle A x^{2}+B x z+C z^{2}\right\rangle$. In the case of $p=5,7,11$ we can go further and say that at least one of the Plücker coordinates can be expressed as a power of the discriminant. When $p=5, v(\Delta)$ and $v\left(\beta_{3}\right)$ determine one another; when $p=7, v(\Delta)$ and $v\left(\beta_{6}\right)$ determine one another; and when $p=11, v(\Delta)$ and $v\left(\beta_{10}\right)$ determine one another.

Explicitly stated, we have the following result:

Theorem 4.1.4. Let $q(x)=A x^{2}+B x z+C z^{2}$ be a quadratic with coefficients in any field of characteristic $p$. Then for $p=5,7,11$ one of the Plücker coordinates of the ideal $\left\langle A x^{2}+B x z+C z^{2}\right\rangle$ is a power of the discriminant $\Delta$ of $q(x)$. When $p=3$, the discriminant appears as a factor in $\beta_{11}$.

Thus, if the valuation of the discriminant deviates from its generic valuation, there will be a corresponding deviation in the valuation of any Plücker coordinate of which $\Delta$ is a factor.

We might then pose the following question:

Question 4.1.5. For what other values of $p$ does Theorem 4.1.4 hold?

It would also be interesting to consider the question in the case of fields of characteristic 2, using an appropriate analogue to the discriminant.

4.2. Patterns in Plücker Coordinates. Having studied and gained a good understanding of the tropical Plücker coordinates of cubics of the form $x^{3}+A x z^{2}+B z^{3}$, it would be useful to know if this allows us to say anything about the Plücker coordinates of tropical elliptic curves in Weierstrass form. The following two propositions show the relationship between the Macaulay matrices of the ideal $\langle f(x, z)\rangle$ and the ideal $\left\langle y^{n-d} z^{d}+f(x, z)\right\rangle$ - that is, the Macaulay matrices of the latter ideal can be expressed in terms of the Macaulay matrices of the former. We will use these results in Sections 4.3 and 5.6.

First, let us make the following definitions:

Definition 4.2.1. Let $f(x, z)$ be a homogeneous polynomial $a_{0} x^{n}+$ $a_{1} x^{n-1} z+\ldots+a_{n} z^{n}$. Then the linear space of the ideal $\langle f(x, z)\rangle$ can be represented by a series of matrices $M_{i}$, where $M_{i}$ represents the degree $n+i-1$ part of the ideal. More explicitly, we have

$$
\begin{aligned}
M_{1} & =\left[\begin{array}{lllc}
a_{0} & a_{1} & \ldots & a_{n}
\end{array}\right] \\
M_{2} & =\left[\begin{array}{ccccc}
a_{0} & a_{1} & \ldots & a_{n} & 0 \\
0 & a_{0} & \ldots & a_{n-1} & a_{n}
\end{array}\right]
\end{aligned}
$$

and so on. $M_{i}$ is the degree $n+i-1$ Macaulay matrix for the ideal $\langle f(x, z)\rangle$.

We will define the matrix $J_{i}$ as follows. Let $I_{i}$ be the $i \times i$ identity matrix, $n$ be the degree of $f(x, z)$ as above, and define $J_{i}$ to be the 
$i \times(n+i)$ matrix whose rightmost $i$ columns are $I_{i}$, and whose other entries are all zeroes. Explicitly, if we were to fix $n=2$ then we would have

$$
\begin{aligned}
& J_{1}=\left[\begin{array}{lll}
0 & 0 & 1
\end{array}\right] \\
& J_{2}=\left[\begin{array}{llll}
0 & 0 & 1 & 0 \\
0 & 0 & 0 & 1
\end{array}\right]
\end{aligned}
$$

and so on.

Proposition 4.2.2. Let $f(x, z)$ be a homogeneous polynomial of degree $n$, let $M_{i}$ and $J_{i}$ be as in Definition 4.2.1 above. The Macaulay matrix of the degree $d$ part of the ideal $\left\langle y^{2} z^{n-2}+f(x, z)\right\rangle$ has the following form:

$$
\left[\begin{array}{ccccccccc}
M_{d} & 0 & J_{d} & 0 & \ldots & 0 & 0 & 0 & 0 \\
0 & M_{d-1} & 0 & J_{d-1} & \ldots & 0 & 0 & 0 & 0 \\
& & \ddots & & \ddots & & & & \\
0 & & \ldots & & & J_{4} & 0 & 0 & 0 \\
0 & & \ldots & & \ddots & 0 & J_{3} & 0 & 0 \\
0 & & \ldots & & \ldots & M_{2} & 0 & J_{2} & 0 \\
0 & & \ldots & & \ldots & 0 & M_{1} & 0 & J_{1}
\end{array}\right]
$$

Proof. Let $N_{d}$ be the matrix representing the degree $d$ part of the linear space of $\left\langle y^{2} z^{n-2}+f(x, z)\right\rangle$ with the rows indexed by $x^{i} y^{j} z^{k}$, where $i+j+k=d-n$, and the columns indexed by all monomials in $x, y, z$ of degree $d$ ordered first by ascending powers of $y$, then by descending powers of $x$. Suppose for some fixed value $D$ of $d$ that the Macaulay matrix has this form. We will show by induction that $N_{D+1}$ must also have this form.

To construct $N_{D+1}$, we multiply each row by $x$, then $y$, then $z$, removing any rows $x^{i_{1}} y^{j_{1}} z^{k_{1}} f, x^{i_{2}} y^{j_{2}} z^{k_{2}} f$ for which $i_{1}=i_{2}, j_{1}=j_{2}$, and $k_{1}=k_{2}$.

First consider the rows of $N_{D+1}$ for which $j=0$. These arise from multplying the rows of $N_{D}$ for which $j=0$ by $x$ and $z$ (and deleting duplicate rows). In the columns indexed by monomials in $x$ and $z$ only, this gives us the degree $D+1$ Macaulay matrix of the ideal $\langle f(x, z)\rangle$. The only other value in these rows is 1 , which falls in columns indexed by $y^{2}$ multiplied by a monomial in $x$ and $z$, beginning with the column corresponding to $y^{2} x^{d-n} z^{n-2}$. This forms the matrix $J_{D+1}$

Now consider the rows for which $j \geq 1$. These are obtained by multiplying all the rows of $N_{D}$ by $y$ and obviously have zeroes in the columns indexed only by $x$ and $z$. The rest of the columns are just the matrix $N_{D}$. Thus if $N_{D}$ has the form given above, so must $N_{D+1}$.

Now all that remains is to show that this is true for some value of $d$. Take $f(x, z)=\sum_{i+j=n} a_{i, j} x^{i} z^{j}$. In degree $n+1$ there are three rows, 
corresponding to multiplying $y^{2} z^{n-2}+f(x, z)$ by $x, z$ and $y$ respectively. Considering first the columns indexed only by $x$ and $z$, the first two rows give us the submatrix below.

$$
\left[\begin{array}{ccccc}
a_{n, 0} & a_{n-1,1} & \ldots & a_{0, n} & 0 \\
0 & a_{n, 0} & \ldots & a_{1, n-1} & a_{0, n}
\end{array}\right]
$$

The only other entries in these rows are due to the $y^{2} z^{n-2}$ term. In the first row we have a 1 in the column indexed by $y^{2} x z^{n-2}$ and in the second we have a 1 in the column indexed by $y^{2} z^{n-1}$. Finally, the third row has entries $a i, j$ in the columns corresponding to $y x^{i} z^{j}$ and a 1 in the column corresponding to $y^{3} z^{n-2}$. Thus it has the required form, as shown below.

$$
\left[\begin{array}{cccc}
M_{2} & 0 & J_{2} & 0 \\
0 & M_{1} & 0 & J_{1}
\end{array}\right]
$$

We can generalise Proposition 4.2.2 in the following way.

Proposition 4.2.3. Let $f(x, z)$ be a homogeneous polynomial of degree $n$, let $M_{i}$ and $J_{i}$ be as in Definition 4.2.1 above. The Macaulay matrix of the degree $d$ part of the ideal $\left\langle y^{m} z^{n-m}+f(x, z)\right\rangle$ has the following form, where $J_{d}$ is in the $(m+1)^{t h}$ column:

$$
\left[\begin{array}{ccccccccc}
M_{d} & 0 & \ldots & 0 & J_{d} & 0 & 0 & \ldots & 0 \\
0 & M_{d-1} & 0 & \ldots & 0 & J_{d-1} & 0 & \ldots & 0 \\
0 & 0 & \ddots & 0 & \ldots & 0 & \ddots & & 0 \\
0 & \ldots & 0 & M_{2} & 0 & 0 & \ldots & J_{2} & 0 \\
0 & \ldots & 0 & 0 & M_{1} & 0 & \ldots & 0 & J_{1}
\end{array}\right]
$$

Proof. We can prove this by modifying the proof of Proposition 4.2.2.

Note 4.2.4. Some brief preliminary calculations show that adding further variables results in similarly structured Macaulay matrices, consisting of blocks that correspond to Macaulay matrices for homogeneous polynomials in two variables. Thus it may be possible to apply certain concepts from the preceding sections to homogeneous polynomials in more than three variables.

4.3. The Quadratic Case in Projective 2-space: Ideals of the Form $\left\langle y^{2}+x^{2}+B x z+C z^{2}\right\rangle$. Since our eventual aim is to study the $j$ invariants of elliptic curves, whose defining equations are hypersurfaces in $\mathbb{P}^{2}$, it makes sense to also investigate quadric hypersurfaces in $\mathbb{P}^{2}$, since the quadratic case is likely to be simpler. Given a quadratic $q(x, z)=x^{2}+B x z+C z^{2}$, we will consider curves of the form $y^{2}+q(x, z)$.

In the quadratic case, the discriminant $\Delta_{2}$ of $q(x, z)=x^{2}+B x z+C z^{2}$ is given by $B^{2}-4 C$. In degree 5 , one of the Plücker coordinates we 
get (using Macaulay2 to calculate the maximal minors of the Macaulay matrix) is

$$
\begin{aligned}
-B^{3} C+4 B C^{2} & =-B C\left(B^{2}-4 C\right) \\
& =-B C \Delta_{2},
\end{aligned}
$$

which clearly vanishes whenever the discriminant vanishes. Thus we get the following:

Proposition 4.3.1. The tropical ideal trop $\left(\left\langle y^{2}+x^{2}+B x z+C z^{2}\right\rangle\right)$ determines the valuation $v\left(\Delta_{2}\right)$ of the discriminant $\Delta_{2}=B^{2}-4 C$ of $x^{2}+B x z+C z^{2}$.

Using the minors and numgens functions in Macaulay2, some simple calculations tell us that there are 64438 non-zero Plücker coordinates in degree 5. Setting $(B, C)=(2,1)$ and $(B, C)=(-10,25)$, which are both roots of the discriminant, in both cases we find that 202 Plücker coordinates vanish. This suggests that the discriminant is a factor of 202 of the Plücker coordinates.

Similarly, if we study the degree 5 Plücker coordinates of the ideal $\left\langle y z+x^{2}+B x z+C z^{2}\right\rangle$ using the same technique as above, we find that there are 32050 non-zero Plücker coordinates, of which 95 vanish when we set $(B, C)=(2,1)$ and $(B, C)=(-10,25)$. Indeed, a quick study of the Plücker coordinates shows that $-B\left(B^{2}-4 C\right)$ is one such vanishing Plücker coordinate.

Thus the discriminant appears as a factor of at least one Plücker coordinate in degree 5 for both $\left\langle y^{2}+x^{2}+B x z+C z^{2}\right\rangle$ and $\left\langle y z+x^{2}+B x z+\right.$ $\left.C z^{2}\right\rangle$. Clearly it cannot arise due to the Sylvester matrix for calculating the resultant of a quadratic $x^{2}+B x+C$ and its derivative $2 x+B$ appearing as a block in the Macaulay matrix, due to the coefficients in the Syvlester matrix as shown below.

$$
\left[\begin{array}{lll}
1 & B & C \\
2 & B & 0 \\
0 & 2 & B
\end{array}\right]
$$

The Macaulay matrix has entries only in the set $\{0,1, B, C\}$ so the entries of 2 mean that the Sylvester matrix can never be a submatrix of it. This is true in higher degrees also; for example in the cubic case, the Sylvester matrix has 3 for some entries.

It is difficult to pinpoint which minors of the Macaulay matrix correspond to the Plücker coordinates involving the discriminant, however another example might give some idea of how factors in the Plücker coordinates of $\left\langle y^{2}+x^{2}+B x z+C z^{2}\right\rangle$ that are not Plücker coordinates of $\left\langle x^{2}+B x z+C z^{2}\right\rangle$ arise. 
Example 4.3.2. Consider the minor given by taking the following submatrix of the degree 5 Macaulay matrix for $\left\langle y^{2}+x^{2}+B x z+C z^{2}\right\rangle$ :

$$
\left[\begin{array}{llllllllll}
C & 0 & 0 & 0 & 0 & 0 & 0 & 0 & 0 & 0 \\
B & C & 0 & 0 & 0 & 0 & 0 & 0 & 1 & 0 \\
1 & B & C & 0 & 0 & 0 & 0 & 1 & 0 & 0 \\
0 & 1 & B & 1 & 0 & 0 & 0 & 0 & 0 & 0 \\
0 & 0 & 0 & 0 & B & C & 0 & 0 & 0 & 0 \\
0 & 0 & 0 & 0 & 1 & B & C & 0 & 0 & 0 \\
0 & 0 & 0 & 0 & 0 & 1 & B & 0 & 0 & 1 \\
0 & 0 & 0 & 0 & 0 & 0 & 0 & C & B & 0 \\
0 & 0 & 0 & C & 0 & 0 & 0 & B & 1 & 0 \\
0 & 0 & 0 & 0 & 0 & 0 & 0 & 0 & 0 & C
\end{array}\right]
$$

Calculating this minor using Macaulay2 yields $-3 B^{5} C^{4}+8 B^{3} C^{5}-$ $4 B C^{6}$, which can be factorised to get

$$
-B C^{4}\left(3 B^{2}-2 C\right)\left(B^{2}-2 C\right) .
$$

You may recognise the factor $B\left(B^{2}-2 C\right)$ as a Plücker coordinate of $\left\langle x^{2}+B x z+C z^{2}\right\rangle$, and indeed the submatrix

$$
\left[\begin{array}{ccc}
B & C & 0 \\
1 & B & C \\
0 & 1 & B
\end{array}\right]
$$

is what gives rise to this factor. Calculating the determinant by hand gives a little more insight into what is going on. We get that the determinant is equal to

$$
\begin{aligned}
B C^{4}( & \left(C-B^{2}\right)\left(B^{2}-2 C\right) \\
& +C\left(B^{2}-2 C\right) \\
& -B^{2}\left(B^{2}-2 C\right) \\
& \left.-B^{2}\left(B^{2}-2 C\right)\right) .
\end{aligned}
$$

Adding, we get $C-B^{2}+C-B^{2}-B^{2}=2 C-3 B^{2}=-\left(3 B^{2}-2 C\right)$. This suggests that the unaccounted-for factors appearing in the Plücker coordinates of $\left\langle y^{2}+f(x, z)\right\rangle$ may occur as linear combinations of products of Plücker coordinates of $\langle f(x, z)\rangle$.

We will note here that this is a special case of the more general equation $A x^{2}+2 B x z+C z^{2}+2 D x y+2 E y z+F y^{2}$ of a conic section, for which $D=E=0$ and $F=1$ (the reason for writing 2 in front of some of the coefficients will become clear soon). A conic section has two different polynomials called discriminants: one, which we will denote by $\Delta$, that is identical to the discriminant of the quadratic $A x^{2}+B x z+C z^{2}$, that over the real numbers determines the shape of the conic section, and another that tells us whether the curve degenerates (e.g. if it becomes 
a double line). We will take a brief look at the latter here, which is given by the determinant of the matrix below.

$$
\left[\begin{array}{lll}
A & B & D \\
B & C & E \\
D & E & F
\end{array}\right]
$$

We get that the discriminant $\Delta^{\prime}$ is $A C F-A E^{2}-B^{2} F+2 B D E-C D^{2}$ (writing, for example, the coefficient of $x z$ as $2 B$ instead of $B$ allows us to avoid fractions in this formula).

In the case where $D=E=0$, we find that

$$
\begin{aligned}
A C F-A E^{2}-B^{2} F+2 B D E-C D^{2} & =A C F-B^{2} F \\
& =-F\left(B^{2}-A C\right) .
\end{aligned}
$$

This vanishes either when $F=0$ or $B^{2}-A C=0$. Tropicalising this gives

$$
\min \{2 v(B), v(A)+v(C)\},
$$

which is the same as the tropicalisation of the discriminant of the quadratic $A x^{2}+2 B x z+C z^{2}$ (i.e., $4\left(B^{2}-A C\right)$ ); thus when $D=E=0$ and $F \neq 0$, each discriminant vanishes if and only if the other does. Since we know that the tropical scheme of $\left\langle y^{2}+x^{2}+B x z+C z^{2}\right\rangle$ determines the valuation of the discriminant of $x^{2}+B x z+C z^{2}$, we get the following result.

Theorem 4.3.3. The valuations of the Plücker coordinates of ideals of the form $\left\langle y^{2}+x^{2}+B x z+C z^{2}\right\rangle$, that is ideals generated by conic sections with $D=E=0, A=F=1$ and coefficients in $\mathbb{C}\{\{t\}\}$, determine the valuation of the discriminant $\Delta=-F\left(B^{2}-A C\right)$.

Here is an example of two quadrics with the same tropicalisation, but different valuations of $\Delta$.

Example 4.3.4. Consider the conics with equations

$$
\begin{aligned}
& c_{1}(x, y, z)=t^{4} x^{2}+2\left(i t^{-1}+1\right) x z+t z^{2}+2 t^{-1} x y+t y^{2} \\
& c_{2}(x, y, z)=t^{4} x^{2}+2\left(t^{-1}+1\right) x z+t z^{2}+2 t^{-1} x y+t y^{2}
\end{aligned}
$$

These both tropicalise to $\min \{4+2 x,-1+x+z, 1+2 z,-1+x+y, 1+2 y\}$. The discriminant is given by $A C F-B^{2} F-C D^{2}$; we have

$$
\begin{aligned}
\Delta\left(c_{1}\right) & =t^{4} \cdot t \cdot(-t)-\left(i t^{-1}+1\right)^{2} \cdot t-t \cdot\left(t^{-1}\right)^{2} \\
& =-2 i-t-t^{6} \\
\Delta\left(c_{2}\right) & =-2 t^{-1}-2-t-t^{6}
\end{aligned}
$$

These do not vanish over $\mathbb{C}\{\{t\}\}$, and $v\left(\Delta\left(c_{1}\right)\right)=0$. However, $\operatorname{trop}\left(\Delta\left(c_{1}\right)\right)=\min \{v(A)+v(C)+v(F), 2 v(B)+v(F), v(C)+2 v(D)\}$, and this gives us $\min \{6,-1,-1\}$, so that the discriminant vanishes tropically and the valuation of the discriminant deviates from the 
expected value. For $c_{2}$, we have $v\left(\Delta\left(c_{2}\right)\right)=-1$ and $\operatorname{trop}\left(\Delta\left(c_{2}\right)\right)=$ $\min \{6,-1,-1\}$; while the discriminant vanishes tropically, its valuation does not deviate from the expected value.

It would be interesting to understand more about the relationship between the Plücker coordinates of the ideal $\left\langle A x^{2}+2 B x z+C z^{2}+\right.$ $\left.2 D x y+2 E y z+F y^{2}\right\rangle$ and the discriminant $\Delta^{\prime}=A C F-A E^{2}-B^{2} F+$ $2 B D E-C D^{2}$.

4.3.1. Characteristic $p$. Since we know that in characteristic $p$ (for $p=3,5,7,11)$ the discriminant appears as a factor in at least one of the Plücker coordinates for $\left\langle x^{2}+B x z+C z^{2}\right\rangle$, and given that some of the Plücker coordinates of $\left\langle y^{2}+x^{2}+B x z+C z^{2}\right\rangle$ are simply products of the Plücker coordinates of $\left\langle x^{2}+B x z+C z^{2}\right\rangle$, we therefore obtain the following:

Proposition 4.3.5. For $B, C$ in some field of characteristic $p$, for $p=3,5,7,11$, the Plücker coordinates of $\left\langle y^{2}+x^{2}+B x z+C z^{2}\right\rangle$ determine the valuation of the discriminant $\Delta=B^{2}-4 C$.

In fact, due to 4.3 .3 we can remove the condition that $p=3,5,7,11$. This means that the valuation of the discriminant does affect the valuations of the Plücker coordinates, so we can further state that:

Theorem 4.3.6. Over fields of characteristic $p \neq 2$, two ideals of the form $\left\langle y^{2}+x^{2}+B x z+C z^{2}\right\rangle$ with the same set-theoretic tropicalisation but different valuated discriminants will have distinct scheme-theoretic tropicalisations.

4.4. Biquadratics and Beyond. When further investigating results which hold for quadratics, it makes sense to generalise to the whole family of ideals of the form $\left\langle x^{2 n}+B x^{n} z^{n}+C z^{2 n}\right\rangle$. First we will take an informal look at what happens in the case of biquadratics, which we define below, then formally prove the results more generally.

A biquadratic is determined by the polynomial $x^{4}+B x^{2} z^{2}+C z^{4}$. If $p, q$ are the roots in any algebraically closed field we can factorise it to $(x+p z)(x-p z)(x+q z)(x-q z)$, which in turn implies that $B=-\left(p^{2}+q^{2}\right)$ and $C=p^{2} q^{2}$. The discriminant of a biquadratic can therefore be written as either $\Delta_{4}=16 C\left(B^{2}-4 C\right)^{2}$ (where $B^{2}-4 C$ is the discriminant of the quadratic in $x^{2}$ ), or in terms of the roots of the polynomial $\Delta_{4}=16 p^{2} q^{2}\left(p^{2}-q^{2}\right)^{2}$.

The first few Macaulay matrices are printed below, for degrees 6, 7, and 8. Using elementary row and column operations, we can rearrange these into a more useful form, as shown, which immediately shows us that the Plücker coordinates of $\left\langle x^{4}+B x^{2} z^{2}+C z^{4}\right\rangle$ depend completely on those of the quadratic $\left\langle x^{2}+B x z+C z^{2}\right\rangle$ : 


$$
\begin{gathered}
{\left[\begin{array}{lllllll}
1 & 0 & B & 0 & C & 0 & 0 \\
0 & 1 & 0 & B & 0 & C & 0 \\
0 & 0 & 1 & 0 & B & 0 & C
\end{array}\right] \rightsquigarrow\left[\begin{array}{llllllll}
1 & B & C & 0 & 0 & 0 & 0 \\
0 & 0 & 0 & 1 & B & C & 0 \\
0 & 0 & 0 & 0 & 1 & B & C
\end{array}\right]} \\
{\left[\begin{array}{lllllllll}
1 & 0 & B & 0 & C & 0 & 0 & 0 \\
0 & 1 & 0 & B & 0 & C & 0 & 0 \\
0 & 0 & 1 & 0 & B & 0 & C & 0 \\
0 & 0 & 0 & 1 & 0 & B & 0 & C
\end{array}\right] \rightsquigarrow\left[\begin{array}{cccccccc}
1 & B & C & 0 & 0 & 0 & 0 & 0 \\
0 & 1 & B & C & 0 & 0 & 0 & 0 \\
0 & 0 & 0 & 0 & 1 & B & C & 0 \\
0 & 0 & 0 & 0 & 0 & 1 & B & C
\end{array}\right]} \\
{\left[\begin{array}{lllllllll}
1 & 0 & B & 0 & C & 0 & 0 & 0 & 0 \\
0 & 1 & 0 & B & 0 & C & 0 & 0 & 0 \\
0 & 0 & 1 & 0 & B & 0 & C & 0 & 0 \\
0 & 0 & 0 & 1 & 0 & B & 0 & C & 0 \\
0 & 0 & 0 & 0 & 1 & 0 & B & 0 & C
\end{array}\right] \rightsquigarrow\left[\begin{array}{ccccccccc}
1 & B & C & 0 & 0 & 0 & 0 & 0 & 0 \\
0 & 1 & B & C & 0 & 0 & 0 & 0 & 0 \\
0 & 0 & 0 & 0 & 1 & B & C & 0 & 0 \\
0 & 0 & 0 & 0 & 0 & 1 & B & C & 0 \\
0 & 0 & 0 & 0 & 0 & 0 & 1 & B & C
\end{array}\right]}
\end{gathered}
$$

To choose a nonzero maximal minor, we must choose columns that form a block diagonal matrix with two square blocks. But these square blocks are maximal minors of the Macaulay matrices for Plücker coordinates of the quadratic $\left\langle x^{2}+B x z+C z^{2}\right\rangle$. Since in the quadratic case, $B^{2}-4 C$ is never a factor of the Plücker coordinates, and $B^{2}-4 C$ is a factor of the discriminant of a biquadratic, it follows that the discriminant of a biquadratic is never a factor of the Plücker coordinates of the ideal generated by a biquadratic polynomial.

Example 4.4.1. We return to the quadratic from Example 4.1.3, and consider this time

$$
Q(x, z)=x^{4}-\left(2 t^{2}+2 t^{3}+t^{4}\right) x^{2} z^{2}+\left(t^{4}+2 t^{5}+t^{6}\right) z^{4} .
$$

$Q$ has four distinct roots, $\pm t$ and $\pm\left(t+t^{2}\right)$ (all with valuation 1 ). The discriminant valuates to 16 , but the generic valuation gives $\min \{12,12,12\}=$ 12 , which again vanishes tropically, giving the repeated root $z=x-1$.

Thus again, we have lost the information telling us that these roots are distinct when passing to the tropical world; what follows will show that in the case of a multiquadratic $Q(x, z)$ we again cannot recover it from the tropical linear space of the ideal $\langle Q(x, z)\rangle$.

Below is a more precise and rigorous exploration of the arguments above, which generalises the result to all ideals of the form $\left\langle x^{2 n}+\right.$ $\left.B x^{n} z^{n}+C z^{2 n}\right\rangle$.

Proposition 4.4.2. The discriminant of a polynomial of the form $x^{2 n}+B x^{n}+C$ is given by the formula

$$
n^{2 n} C^{n-1}\left(B^{2}-4 C\right)^{2 n} \text {. }
$$

Proof. First we note that the discriminant of a polynomial $f(x)$ of degree $n$ in one variable can be characterised as the following product, 
which makes obvious that $\Delta$ vanishes if and only if there is a repeated root. For $1 \leq j<i \leq n$,

$$
\Delta(p(x))=\prod_{i, j}\left(p_{i}-p_{j}\right)^{2},
$$

where the $p_{i}$ are the roots of $f(x)=0$. Thus the discriminant of the product of two polynomials $f(x)$ and $g(x)$ can be given by the formula

$$
\Delta(f(x) \cdot g(x))=\Delta(f(x)) \cdot \Delta(g(x)) \cdot \prod_{i} \prod_{j}\left(p_{i}-q_{j}\right)^{2},
$$

where the $p_{i}$ are the roots of $f(x)$, the $q_{i}$ are the roots of $g(x)$, and $1 \leq i, j \leq 0$.

Next, we note that the polynomial $m(x)=x^{2} n+B x^{n}+C$ factorises into $\left(x^{n}-p^{n}\right)\left(x^{n}-q^{n}\right)$, so that the roots of $m(x)$ are $\omega^{i} p, \omega^{j} q$, where $\omega$ is the primitive $n^{\text {th }}$ root of unity, and $0 \leq i, j \leq n-1$.

Another characterisation of the discriminant of a polynomial $f(x)$ is the formula

$$
\Delta(f(x))=(-1)^{\frac{n(n-1)}{2}} \operatorname{Res}\left(f(x), f^{\prime}(x)\right) .
$$

(The sign was historically chosen so that for polynomials with real coefficients, the discriminant is positive when all the roots are real.) We will find the discriminant of the polynomial $f(x)=x^{n}-p^{n}$ from the Sylvester matrix of $f(x)$ and $f^{\prime}(x)$, which is as follows:

$$
\left[\begin{array}{cccccccccc}
1 & 0 & 0 & \ldots & 0 & 0 & -p^{n} & 0 & \ldots & 0 \\
0 & 1 & 0 & \ldots & 0 & 0 & 0 & -p^{n} & \ldots & 0 \\
\vdots & & & & & & & & & \vdots \\
0 & 0 & 0 & \ldots & 1 & 0 & 0 & 0 & \ldots & -p^{n} \\
n & 0 & \ldots & & & & & & & 0 \\
0 & n & 0 & \ldots & & & & & & 0 \\
\vdots & & & & & & & & \vdots \\
0 & \ldots & & & & n & 0 & 0 & \ldots & 0
\end{array}\right]
$$

Expanding first along the bottom $n$ rows, it's clear that the determinant of this matrix is

$$
n^{n} \cdot p^{n(n-1)}
$$

Thus the discriminant of $x^{n}-p^{n}$ is

$$
(-1)^{\frac{n(n-1)}{2}} n^{n} p^{n(n-1)}
$$

Using the formula given above, the discriminant of $\left(x^{n}-p^{n}\right)\left(x^{n}-q^{n}\right)$ is therefore

$$
n^{n} p^{n(n-1)} \cdot n^{n} q^{n(n-1)} \cdot \prod_{i} \prod_{j}\left(\omega^{i} p-\omega^{j} q\right)^{2}
$$

where $\omega$ is the primitive $n^{t} h$ root of unity and $0 \leq i, j \leq n-1$. 
We know that

$$
\begin{aligned}
p^{n}-q^{n} & =(p-q)(p-\omega q)\left(p-\omega^{2} q\right) \ldots\left(p-\omega^{n-1} q\right) \\
& =(\omega p-q)(\omega p-\omega q)\left(\omega p-\omega^{2} q\right) \ldots\left(\omega p-\omega^{n-1} q\right) \\
& =\left(\omega^{2} p-q\right)\left(\omega^{2} p-\omega q\right)\left(\omega^{2} p-\omega^{2} q\right) \ldots\left(\omega^{2} p-\omega^{n-1} q\right)
\end{aligned}
$$

and so the product

$$
\prod_{i} \prod_{j}\left(\omega^{i} p-\omega^{j} q\right)^{2}=\left(p^{n}-q^{n}\right)^{n} .
$$

Putting all of this together gives us the result. For a polynomial

$$
x^{2 n}+B x^{n}+C=\left(x^{n}-p^{n}\right)\left(x^{n}-q^{n}\right)=x^{2 n}-\left(p^{n}+q^{n}\right) x^{n}+p^{n} q^{n},
$$

the discriminant is equal to

$$
n^{2 n}\left(p^{n} q^{n}\right)^{(n-1)}\left(p^{n}-q^{n}\right)^{2 n}=n^{2 n} C^{(n-1)}\left(B^{2}-4 C\right)^{2 n} .
$$

It appears that the Plücker coordinates obtained from the central square of the Macaulay matrix at each degree can be described explicitly as shown below in Proposition 4.4.3, and indeed the roots of all Plücker coordinates of $\left\langle x^{2 n}+B x^{n} z^{n}+C z^{2 n}\right\rangle$ are entirely determined by the roots of the Plücker coordinates of $\left\langle x^{2}+B x z+C z^{2}\right\rangle$.

Proposition 4.4.3. The Plücker coordinates $\beta_{k}^{n}$ of the $k=(\text { in }+j)^{t h}$ degree part of the ideal generated by $x^{2 n}+B x^{n} z^{n}+C z^{2 n}$ are given by the formula

$$
\beta_{k}^{n}=\left(\beta_{i}\right)^{n-\bar{j}+1} \cdot\left(\beta_{i+1}\right)^{\bar{j}+1},
$$

where $\bar{j}=j \bmod n$, and $\beta_{i}$ is the degree $i$ Plücker coordinate of $\left\langle x^{2}+\right.$ $\left.B x z+C z^{2}\right\rangle$ obtained from the square submatrix of the degree $i$ Macaulay matrix with $B$ for all entries along the leading diagonal.

Furthermore, all Plücker coordinates for the ideal $\left\langle x^{2 n}+B x^{n} z^{n}+\right.$ $\left.C z^{2 n}\right\rangle$ are products of lower-degree Plücker coordinates of the ideal $\left\langle x^{2}+B x z+C z^{2}\right\rangle$.

Proof. For any degree $k$, first take the minor of the Macaulay matrix which has $B$ for every entry along the main diagonal (this will give us the highest-degree Plücker coordinate). Apart from the main diagonal, the matrix has two other diagonal bands of entries - one starting from the first row in the $(n+1)^{t h}$ column whose every entry is $C$ and the other starting from the $(n+1)^{t h}$ row in the first column whose every entry is 1 .

We will use a series of elementary row operations to rearrange this $k \times k$ matrix into a form that makes calculating the determinant very easy. Label each row (from top to bottom) and each column (from left to right) with numbers $0, \ldots, k-1$, then reduce to their equivalence class $\bar{k} \bmod n$. For each $\bar{k}=\overline{0}, \ldots, \overline{n-1}$, take every row and column 
with that label and rearrange the matrix into a block diagonal matrix as shown below. Note that this will always involve switching an even number of rows and columns, thus the sign of the determinant will not change.

$$
\left[\begin{array}{ccccc}
\overline{0} & 0 & \ldots & 0 & 0 \\
0 & \overline{1} & \ldots & 0 & 0 \\
& & \ddots & & \\
0 & 0 & \ldots & \overline{n-2} & 0 \\
0 & 0 & \ldots & 0 & \overline{n-1}
\end{array}\right]
$$

For $0 \leq \bar{k} \leq j$, we get a block that forms a $(i+1) \times(i+1)$ tridiagonal matrix whose entries are $B$ down the main diagonal, and 1 and $C$ below and above the main diagonal respectively, the determinant of which is $\beta_{i+1}$, the highest degree Plücker coordinate in degree $i+1$ of the ideal generated by a quadratic. Similarly for $j<\bar{k} \leq n-1$ we get a block that forms a tridiagonal $i \times i$ matrix with determinant $\beta_{i}$.

Thus, when we calculate the determinant of the above matrix, we get

$$
\left(\beta_{i+1}\right)^{\bar{j}+1} \cdot\left(\beta_{i}\right)^{n-\bar{j}-1}
$$

and the first part of the proposition is proved.

Now, we write out the Macaulay matrix as a whole and use the same method. This results in a block diagonal matrix with $n$ rectangular blocks. To choose columns that give us a nonzero maximal minor, we must choose so that we obtain a block diagonal matrix with $n$ square blocks. These blocks all correspond to maximal minors of the Macaulay matrices for the ideal $\left\langle x^{2}+B x z+C z^{2}\right\rangle$, thus we have proved the second statement.

Proposition 4.4.3 can be generalised as follows for when $f$ has degree greater than or equal to 2 .

Theorem 4.4.4. Let $f(x, z)$ be a homogeneous polynomial of degree $d$, and let $F(x, z)$ be the homogeneous polynomial obtained from $f$ by applying the map $x \mapsto x^{n}, z \mapsto z^{n}$. All Plücker coordinates of $\langle F(x, z)\rangle$ can be written as products of Plücker coordinates of $\langle f(x, z)\rangle$.

Proof. Following the method given in the proof of Proposition 4.4.3 yields the result.

Corollary 4.4.5. For all $n \in \mathbb{N}$, the discriminant $\Delta_{n}=n^{n} C^{n-1}\left(B^{2}-\right.$ $4 C)^{2 n}$ of the polynomial $x^{2 n}+B x^{n} z^{n}+C z^{2 n}$ is not a factor of the Plücker coordinates of $\left\langle x^{2 n}+B x^{n} z^{n}+C z^{2 n}\right\rangle$.

Proof. Since it has already been proven that the discriminant of the quadratic $x^{2}+B x z+C z^{2}$ never appears as a factor in the Plücker coordinates of the ideal $\left\langle x^{2}+B x z+C z^{2}\right\rangle$, this follows directly from Proposition 4.4.3. 
Proposition 4.4.6. Suppose $f(x, z)$ is a homogeneous polynomial in $\mathbb{C}\{\{t\}\}[x, z]$ and $F(x, z)$ is the polynomial obtained from $f$ by applying the map $x \mapsto x^{n}, z \mapsto z^{n}$. Suppose that the valuation of the discriminant of $f(x, z)$ is not determined by the valuations of the Plücker coordinates of the ideal $\langle f(x, z)\rangle$. Then the valuation of the discriminant of $F(x, z)$ is not determined by the Plücker coordinates of the ideal $\langle F(x, z)\rangle$.

Before we can prove Proposition 4.4.6, we need another result along the lines of Proposition 4.4.2.

Lemma 4.4.7. Let $f(x, z)$ and $F(x, z)$ be as above, and let $\Delta_{d}$ and $\Delta_{n d}$ be the respective discriminants of $f(x, z)$ and $F(x, z)$. Then

$$
\Delta_{n d}=0 \Leftrightarrow \Delta_{d}=0 .
$$

Proof. Let $\alpha_{1}, \ldots, \alpha_{d}$ denote the roots of $f(x, z)$. Then $F(x, z)$ has roots $\omega^{j} \alpha_{i}(i=1, \ldots, d, j=0, \ldots, n-1)$, where $\omega$ is the primitive $n^{t h}$ root of unity. Clearly for all $\alpha_{i}, \omega^{j} \alpha_{i}=\omega^{k} \alpha_{i}$ if and only if $j=k$, thus the $n^{t h}$ roots of each $\alpha_{i}$ cannot yield multiple roots. The only way for $F(x, z)$ to have multiple roots, therefore, is if $\alpha_{p}=\alpha_{q}$ for some $1 \leq p, q \leq d$, in which case $f(x, z)$ must also have a repeated root. The opposite implication is obvious.

The discriminant of $F(x, z)$ is a power of the discriminant of $f(x, z)$, up to multiplication by a monomial, as the following proposition shows:

Proposition 4.4.8. Let $f(x, z), F(x, z), \Delta_{d}$ and $\Delta_{n d}$ be as above. Suppose $f(x, z)$ has constant term $a_{0}$. Then $\Delta_{d}$ is always a factor of $\Delta_{n d}$, and we have

$$
\Delta_{n d}=n^{n d} a_{0}^{n-1} \cdot\left(\Delta_{d}\right)^{n} .
$$

Proof. First we will define the Sylvester matrix of $F$ as follows: the first row consists of the coeffients of $x^{i} z^{n d-1}$ organised in descending powers of $x$, followed by $n d-2$ zeroes. Each subsequent row, until the $(n d-1)^{t h}$ row, shifts the nonzero entries one step to the right. The $n d^{\text {th }}$ row consists of the coefficients of the derivative $F^{\prime}$ of $F$, followed by $n d-1$ zeroes. The subsequent rows, up to the $(2 n d-1)^{t h}$ row, shift the nonzero entries one step to the right each time. The determinant of this $(2 n d-1) \times(2 n d-1)$ matrix is defined to be the resultant $\operatorname{Res}\left(F, F^{\prime}\right)$ of $F$ and $F^{\prime}$. Multiplying this by $(-1)^{\frac{n d(n d-1)}{2}} \frac{1}{a_{n d}}$, where $a_{n d}$ is the coefficient of $x^{\text {nd }}$, gives the discriminant of $F$.

Applying the method of Proposition 4.4.3 to the Sylvester matrix for $F(x, z)$ results in a block diagonal matrix with one block resembling the Sylvester matrix for $f(x, z)$, but with each coefficient in the bottom $d$ rows multipluied by $n$. This block has determinant $n^{d} \cdot \operatorname{Res}\left(f, f^{\prime}\right)$ (by basic properties of elementary row operations and discriminants). The other $n-1$ blocks are similar to the above, but with an additional row and column, and have determinant $a_{0} n^{d} \cdot \operatorname{Res}\left(f, f^{\prime}\right)$. Multiplying by the appropriate scaling factor gives the result. 
Proof of Corollary 4.4.6. By Theorem 4.4.4, we know that the discriminant of $f(x, z)$ cannot be a factor of the Plücker coordinates of $\langle F(x, z)\rangle$, and by Proposition 4.4 .8 the discriminant of $F(x, z)$ is a power of the discriminant of $f(x, z)$, thus the discriminant of $F(x, z)$ cannot be a factor of the Plücker coordinates of $\langle F(x, z)\rangle$. Thus the Plücker coordinates of $\langle F(x, z)\rangle$ cannot determine the valuation of the discriminant of $F(x, z)$.

Taken together, these results show that if we understand the Plücker coodinates of the ideal $\langle f(x, z)\rangle$ generated by a homogeneous polynomial in two variables, we can easily extend our understanding to a whole family of ideals. However, we must note that the latter part of the following question remains unanswered.

Question 4.4.9. For $n>2$, are the Plücker coordinates for the ideal $\left\langle\sum_{i=0}^{n} a_{i} x^{i} z^{n-i}\right\rangle$ described entirely by a single recurrence relation? If not, is there a simple way of describing them all?

We know that in general the Plücker coordinates cannot be fully described by a single recurrence relation even for cubics, but it would be interesting to know if there is some convenient way to describe them. Some preliminary work would suggest that, for a quartic $A x^{4}+B x^{3} z+$ $C x^{2} z^{2}+D x z^{3}+E z^{4}$, the Plücker coordinates that do not arise from minors of the Macaulay matrix containing a complete diagonal in any of $A, B, C, D, E$ are linear combinations of products of lower-degree Plücker coordinates.

4.4.1. The Multiquadratic Case in Characteristic $p$. We saw in Section 4.1.4 that in characteristic $p$ for $p=3,5,7,11$, the valuation of the discriminant of a quadratic is not independent of the valuations of the Plücker coordinates of the ideal it generates. In the previous section, we saw that the valuation of the discriminant of the multiquadratic $A x^{2 n}+B x^{n} z^{n}+C z^{2 n}$ depends on the valuation of the disriminant of the quadratic $A x^{2}+B x z+C z^{2}$, and that the valuations of the Plücker coordinates of the former also depend on the latter. Thus for $p=3,5,7,11$ we have the following result:

Theorem 4.4.10. Let $p(x)=A x^{2 n}+B x^{n} z^{n}+C z^{2 n}$ be a multiquadratic with coefficients in any field of characteristic $p$, for $p=3,5,7,11$. Then the valuations of the Plücker coordinates of the ideal $\left\langle A x^{2 n}+B x^{n} z^{n}+\right.$ $\left.C z^{2 n}\right\rangle$ determine the valuation of its discriminant.

Again, we might ask for which other values of $p$ this holds.

4.5. The Multiquadratic Case in Projective 2-space: Ideals of the Form $\left\langle y^{2 n}+x^{2 n}+B x^{n} z^{n}+C z^{2 n}\right\rangle$. We have seen in Section 4.4 that the Plücker coordinates of multiquadratics of the form $x^{2 n}+B x^{n} z^{n}+$ $C z^{2 n}$ can be described entirely by the Plücker coordinates of the ideal generated by a quadratic $\left\langle x^{2}+B x z+C z^{2}\right\rangle$, and in Section 4.3 we 
saw that the quadratic discriminant $B^{2}-4 C$ appears as a factor in the degree 5 Plücker coordinates of $\left\langle y^{2}+x^{2}+B x z+C z^{2}\right\rangle$. It is therefore natural to ask whether the valuation of the discriminant of $x^{2 n}+B x^{n} z^{n}+C z^{2 n}$ depends on the valuations of the Plücker coordinates of $\left\langle y^{2 n}+x^{2 n}+B x^{n} z^{n}+C z^{2 n}\right\rangle$.

We will use biquadratics as an example, to show that $B^{2}-4 C$ (which is a factor of the discriminant of a biquadratic) must also be a factor of the Plücker coordinates of $\left\langle y^{4}+x^{4}+B x^{2} z^{2}+C z^{4}\right\rangle$, so that the valuation of the discriminant of $x^{4}+B x^{2} z^{2}+C z^{4}$ does depends on the valuations of the Plücker coordinates, which provides evidence that the following more general statement is likely to be true:

Conjecture 4.5.1. For any ideal of the form $I=\left\langle y^{2 n}+x^{2 n}+B x^{n} z^{n}+\right.$ $\left.C z^{2 n}\right\rangle, B^{2}-4 C$ is a factor of both the discriminant $\Delta$ of $x^{2 n}+B x^{n} z^{n}+$ $C z^{2 n}$ and some Plücker coordinate of the ideal, thus the valuation of $\Delta$ is not independent of the valuation of the Plücker coordinates of $I$.

Consider the ideal $\langle f(x, y, z)\rangle=\left\langle y^{4}+x^{4}+B x^{2} z^{2}+C z^{4}\right\rangle$. We will construct the Macaulay matrix in each degree $d$ as follows. Label the columns by all monomials $x^{i} z^{j} y^{k}$ in degree $d$, ordered from the left by ascending powers of $y$ then descending powers of $x$, so that they begin

$$
x^{d}, x^{d-1} z, \ldots, z^{d}, y x^{d-1}, y x^{d-2} z, \ldots, y^{d}
$$

Then label the rows similarly, by the product of $f$ by all monomials $x^{i} z^{j} y^{k}$ of degree $d-4$, again ordered from the top by ascending powers of $y$ and descending powers of $x$.

Let $M_{n}$ be the Macaulay matrix for $\left\langle x^{4}+B x^{2} z^{2}+C z^{4}\right\rangle$ in degree $n$, and let $I_{n}$ be the $n \times n$ identity matrix. The Macaulay matrix in degree $d$ for $\left\langle y^{4}+x^{4}+B x^{2} z^{2}+C z^{4}\right\rangle$ has the following form (see Section 4.2 for more on this):

$$
\left[\begin{array}{ccccccccccc}
M_{d} & 0 & 0 & 0 & I_{d-3} & 0 & \ldots & 0 & 0 & 0 & 0 \\
0 & M_{d-1} & 0 & 0 & 0 & I_{d-4} & \ldots & 0 & 0 & 0 & 0 \\
\vdots & & \ddots & & & & \ddots & & & \vdots & \\
0 & 0 & & \ldots & & \ldots & M_{4} & 0 & 0 & 0 & I_{1}
\end{array}\right]
$$

Now, we know from Proposition 4.4.3 that the $M_{n}$ can be rearranged via permutations of the rows and columns into a block diagonal matrix whose blocks consist of $M_{n}^{\prime}$, the Macaulay matrices in degree $n$ for the ideal $\left\langle x^{2}+B x z+C z^{2}\right\rangle$. Rearranging the rows and columns in this way does not change the $I_{n}$, as we see in the example below. 
Example 4.5.2. Take the following submatrix of the Macaulay matrix, with all rows of only zeros omitted:

$$
\left[\begin{array}{lllllll}
1 & 0 & 0 & 0 & 0 & 0 & 0 \\
0 & 1 & 0 & 0 & 0 & 0 & 0 \\
0 & 0 & 1 & 0 & 0 & 0 & 0 \\
0 & 0 & 0 & 1 & 0 & 0 & 0 \\
0 & 0 & 0 & 0 & 1 & 0 & 0 \\
0 & 0 & 0 & 0 & 0 & 1 & 0 \\
0 & 0 & 0 & 0 & 0 & 0 & 1 \\
1 & 0 & B & 0 & C & 0 & 0 \\
0 & 1 & 0 & B & 0 & C & 0 \\
0 & 0 & 1 & 0 & B & 0 & C
\end{array}\right]
$$

Rearranging this, we get the following:

$$
\left[\begin{array}{lllllll}
1 & 0 & 0 & 0 & 0 & 0 & 0 \\
0 & 0 & 0 & 0 & 1 & 0 & 0 \\
0 & 1 & 0 & 0 & 0 & 0 & 0 \\
0 & 0 & 0 & 0 & 0 & 1 & 0 \\
0 & 0 & 1 & 0 & 0 & 0 & 0 \\
0 & 0 & 0 & 0 & 0 & 0 & 1 \\
0 & 0 & 0 & 1 & 0 & 0 & 0 \\
1 & B & C & 0 & 0 & 0 & 0 \\
0 & 1 & B & C & 0 & 0 & 0 \\
0 & 0 & 0 & 0 & 1 & B & C
\end{array}\right]
$$

Now, we consider the following submatrix of the Macaulay matrix, taking the rows including the top seven rows of the previous matrix and omitting any columns consisting only of zeros:

$$
\left[\begin{array}{llllllllllllllllll}
1 & 0 & B & 0 & C & 0 & 0 & 0 & 0 & 0 & 0 & 1 & 0 & 0 & 0 & 0 & 0 & 0 \\
0 & 1 & 0 & B & 0 & C & 0 & 0 & 0 & 0 & 0 & 0 & 0 & 0 & 0 & 1 & 0 & 0 \\
0 & 0 & 1 & 0 & B & 0 & C & 0 & 0 & 0 & 0 & 0 & 1 & 0 & 0 & 0 & 0 & 0 \\
0 & 0 & 0 & 1 & 0 & B & 0 & C & 0 & 0 & 0 & 0 & 0 & 0 & 0 & 0 & 1 & 0 \\
0 & 0 & 0 & 0 & 1 & 0 & B & 0 & C & 0 & 0 & 0 & 0 & 1 & 0 & 0 & 0 & 0 \\
0 & 0 & 0 & 0 & 0 & 1 & 0 & B & 0 & C & 0 & 0 & 0 & 0 & 0 & 0 & 0 & 1 \\
0 & 0 & 0 & 0 & 0 & 0 & 1 & 0 & B & 0 & C & 0 & 0 & 0 & 1 & 0 & 0 & 0
\end{array}\right]
$$

We rearrange this to get:

$$
\left[\begin{array}{llllllllllllllllll}
1 & B & C & 0 & 0 & 0 & 0 & 0 & 0 & 0 & 0 & 1 & 0 & 0 & 0 & 0 & 0 & 0 \\
0 & 1 & B & C & 0 & 0 & 0 & 0 & 0 & 0 & 0 & 0 & 1 & 0 & 0 & 0 & 0 & 0 \\
0 & 0 & 1 & B & C & 0 & 0 & 0 & 0 & 0 & 0 & 0 & 0 & 1 & 0 & 0 & 0 & 0 \\
0 & 0 & 0 & 1 & B & C & 0 & 0 & 0 & 0 & 0 & 0 & 0 & 0 & 1 & 0 & 0 & 0 \\
0 & 0 & 0 & 0 & 0 & 0 & 1 & B & C & 0 & 0 & 0 & 0 & 0 & 0 & 1 & 0 & 0 \\
0 & 0 & 0 & 0 & 0 & 0 & 0 & 1 & B & C & 0 & 0 & 0 & 0 & 0 & 0 & 1 & 0 \\
0 & 0 & 0 & 0 & 0 & 0 & 0 & 0 & 1 & B & C & 0 & 0 & 0 & 0 & 0 & 0 & 1
\end{array}\right]
$$


Thus rearranging the blocks $M_{n}$ into the blocks $M_{n}^{\prime}$ does not affect the pattern described above.

Now, this means that we can further break down the pattern of the Macaulay matrix in degree $d=2 k$ for some $k$ of $\left\langle y^{4}+x^{4}+B x^{2} z^{2}+C z^{4}\right\rangle$ into the following form (where 0 denotes a block consisting of zeros):

$$
\left[\begin{array}{cccccccccccc}
M_{k}^{\prime} & 0 & 0 & 0 & 0 & 0 & 0 & 0 & I_{k-1} & 0 & \ldots & 0 \\
0 & M_{k-1}^{\prime} & 0 & 0 & 0 & 0 & 0 & 0 & 0 & I_{k-2} & \ldots & 0 \\
0 & 0 & M_{k-1}^{\prime} & 0 & 0 & 0 & 0 & 0 & 0 & 0 & \ddots & 0 \\
0 & 0 & 0 & M_{k-1}^{\prime} & 0 & 0 & 0 & 0 & 0 & 0 & & 0 \\
0 & 0 & 0 & 0 & M_{k-1}^{\prime} & 0 & 0 & 0 & 0 & 0 & & 0 \\
0 & 0 & 0 & 0 & 0 & M_{k-2}^{\prime} & 0 & 0 & 0 & 0 & & 0 \\
& & & & & & \ddots & & & & & \vdots \\
0 & & \ldots & & & & & & & \ldots & & I_{1}
\end{array}\right]
$$

There are at most four copies of each block $M_{i}^{\prime}$ and the corresponding identity block $I_{i-1}$; grouping together the rows and columns containing the first, second, third and fourth copy of each in the manner of the proof of 4.4 .3 gives us the following.

Proposition 4.5.3. Let $N_{k}^{\prime}$ be the Macaulay matrix in degree $k$ for $\left\langle y^{2}+x^{2}+B x z+C z^{2}\right\rangle$ with all columns of only zeros omitted. Then (for $k \geq 3$ ) the Macaulay matrix $N_{2 k}$ in degree $2 k$ is given by

$$
\left[\begin{array}{cccc}
N_{k}^{\prime} & 0 & 0 & 0 \\
0 & N_{k-1}^{\prime} & 0 & 0 \\
0 & 0 & N_{k-1}^{\prime} & 0 \\
0 & 0 & 0 & N_{k-1}^{\prime}
\end{array}\right]
$$

and in degree $2 k+1$ it is given by

$$
\left[\begin{array}{cccc}
N_{k}^{\prime} & 0 & 0 & 0 \\
0 & N_{k}^{\prime} & 0 & 0 \\
0 & 0 & N_{k}^{\prime} & 0 \\
0 & 0 & 0 & N_{k-1}^{\prime}
\end{array}\right]
$$

In particular, this means that all Plücker coordinates of $\left\langle y^{4}+x^{4}+\right.$ $\left.B x^{2} z^{2}+C z^{4}\right\rangle$ are products of lower degree Plücker coordinates of $\left\langle y^{2}+\right.$ $\left.x^{2}+B x z+C z^{2}\right\rangle$.

To make this more clear we will first look at the Macaulay matrices in degrees 4, 5, and 6 as examples, before proving the statement more formally. In degree 4 we simply get the matrix

$$
\left[\begin{array}{llllllll}
1 & 0 & B & 0 & C & 0 & \ldots & 1
\end{array}\right]
$$

which is easily rearranged into

$$
\left[\begin{array}{llll}
1 & B & C & 1
\end{array}\right]=\left[\begin{array}{ll}
M_{2}^{\prime} & I_{1}
\end{array}\right]=\left[N_{2}^{\prime}\right]
$$


In degree 5 , omitting the columns of only zeroes, we get

$$
\left[\begin{array}{llllllllllll}
1 & 0 & B & 0 & C & 0 & 0 & 0 & 0 & 1 & 0 & 0 \\
0 & 1 & 0 & B & 0 & C & 0 & 0 & 0 & 0 & 1 & 0 \\
0 & 0 & 0 & 0 & 0 & 0 & 1 & B & C & 0 & 0 & 1
\end{array}\right]
$$

which we rearrange into the following form:

$$
\left[\begin{array}{llllllllllll}
1 & B & C & 1 & 0 & 0 & 0 & 0 & 0 & 0 & 0 & 0 \\
0 & 0 & 0 & 0 & 1 & B & C & 1 & 0 & 0 & 0 & 0 \\
0 & 0 & 0 & 0 & 0 & 0 & 0 & 0 & 1 & B & C & 1
\end{array}\right]
$$

This is clearly the matrix

$$
\left[\begin{array}{ccc}
N_{2}^{\prime} & 0 & 0 \\
0 & N_{2}^{\prime} & 0 \\
0 & 0 & N_{2}^{\prime}
\end{array}\right]
$$

Finally, degree 5 gives us the following Macaulay matrix, with six additional columns on the right forming $I_{6}$ :

$$
\left[\begin{array}{llllllllllllllll}
1 & 0 & B & 0 & C & 0 & 0 & 0 & 0 & 0 & 0 & 0 & 0 & 0 & 0 & 0 \\
0 & 1 & 0 & B & 0 & C & 0 & 0 & 0 & 0 & 0 & 0 & 0 & 0 & 0 & 0 \\
0 & 0 & 1 & 0 & B & 0 & C & 0 & 0 & 0 & 0 & 0 & 0 & 0 & 0 & 0 \\
0 & 0 & 0 & 0 & 0 & 0 & 0 & 1 & 0 & B & 0 & C & 0 & 0 & 0 & 0 \\
0 & 0 & 0 & 0 & 0 & 0 & 0 & 0 & 1 & 0 & B & 0 & C & 0 & 0 & 0 \\
0 & 0 & 0 & 0 & 0 & 0 & 0 & 0 & 0 & 0 & 0 & 0 & 0 & 1 & B & C
\end{array}\right]
$$

We can rearrange these columns (preserving the $I_{6}$ ) to get

$$
\left[\begin{array}{llllllllllllllll}
1 & B & C & 0 & 0 & 0 & 0 & 0 & 0 & 0 & 0 & 0 & 0 & 0 & 0 & 0 \\
0 & 1 & B & C & 0 & 0 & 0 & 0 & 0 & 0 & 0 & 0 & 0 & 0 & 0 & 0 \\
0 & 0 & 0 & 0 & 1 & B & C & 0 & 0 & 0 & 0 & 0 & 0 & 0 & 0 & 0 \\
0 & 0 & 0 & 0 & 0 & 0 & 0 & 1 & B & C & 0 & 0 & 0 & 0 & 0 & 0 \\
0 & 0 & 0 & 0 & 0 & 0 & 0 & 0 & 0 & 0 & 1 & B & C & 0 & 0 & 0 \\
0 & 0 & 0 & 0 & 0 & 0 & 0 & 0 & 0 & 0 & 0 & 0 & 0 & 1 & B & C
\end{array}\right]
$$

Inserting appropriate columns of $I_{6}$ after each block therefore allows us to rearrange the degree 5 Macaulay matrix as

$$
\left[\begin{array}{cccc}
N_{3}^{\prime} & 0 & 0 & 0 \\
0 & N_{2}^{\prime} & 0 & 0 \\
0 & 0 & N_{2}^{\prime} & 0 \\
0 & 0 & 0 & N_{2}^{\prime}
\end{array}\right]
$$

With Proposition 4.5.3, along with Theorem 4.3.3 we now have the following result:

Theorem 4.5.4. The valuations of the Plücker coordinates of $\left\langle y^{4}+\right.$ $\left.x^{4}+B x^{2} z^{2}+C z^{4}\right\rangle$ determine the valuation of the discriminant of $x^{4}+B x^{2} z^{2}+C z^{4}$.

We should be able to prove Conjecture 4.5.1 in a similar way. 


\section{Generalising to Degree 3}

In this section we turn our attention to cubics, and ask whether the valuation of the discriminant of a cubic polynomial $p(x, z)$ is determined by the tropical ideal $\operatorname{trop}(\langle p(x, z)\rangle)$. In Section 5.3 we will show that, over fields of characteristic 0 , for cubics of the form $x^{3}+A x z^{2}+B z^{3}$ the valuation of the discriminant is not determined by the valuations of the Plücker coordinates, a result which can be generalised to all trinomials of the form $x^{n}+A x z^{n-1}+B z^{n}$. We show that, analogously to the quadratic case, when working over fields of characteristic $p$, the

5.1. Cubics of the Form $x^{3}+A x z^{2}+B z^{3}$. For motivation, we will begin with an example along the lines of Example 4.1.3, considering a plane cubic and its corresponding elliptic curve.

Example 5.1.1. Consider the homogeneous cubic

$$
c(x, z)=x^{3}-\left(3 t^{2}+3 t^{3}+t^{4}\right) x z^{2}+\left(2 t^{3}+3 t^{4}+t^{5}\right) z^{3},
$$

which has distinct roots $t, t+t^{2},-2 t-t^{2}$ in the field of Puiseux series. The discriminant of a cubic of the form $x^{3}+A x z^{2}+B z^{3}$ is given by $\Delta=-4 A^{3}-27 B^{2}$; for $c$ we get

$$
\Delta=81 t^{8}+162 t^{9}+117 t^{10}+36 t^{11}+4 t^{12} .
$$

Thus the valuation of the discriminant of $c, v(\Delta(c))=8$. However, the generic valuation gives us

$$
\operatorname{trop}(\Delta)(c)=\min \{3 v(A), 2 v(B)\}=\min \{6,6\}=6,
$$

which vanishes tropically, since the minimum is attained twice.

Now, consider the elliptic curve $E_{c}$ given by

$$
\begin{aligned}
E_{c} & =y^{2} z-c \\
& =y^{2} z-x^{3}+\left(3 t^{2}+3 t^{3}+t^{4}\right) x z^{2}-\left(2 t^{3}+3 t^{4}+t^{5}\right) z^{3} .
\end{aligned}
$$

The $j$-invariant of an elliptic curve of the form $y^{2} z-x^{3}-A x z^{2}-B z^{3}$ is given by the formula

$$
j=1728 \cdot \frac{4 A^{3}}{-\Delta}
$$

where $\Delta$ is the discriminant of $c$; the valuation $v\left(j\left(E_{c}\right)\right)=6-8=-2$. However, tropicalising the formula for the $j$-invariant results in

$$
\begin{aligned}
\operatorname{trop}(j) & =3 v(A)-\min \{3 v(A), 2 v(B)\} \\
& =6-\min \{6,6\}=0 .
\end{aligned}
$$

Our cubic above, $c(x, z)$, corresponds to an elliptic curve whose cycle "disappears" under the tropicalisation map, and we can't determine its tropical $j$-invariant from the cycle length. Just as information about the discriminant is "lost" under certain conditions, so is information about the $j$-invariant; we would like to know whether some of this information is encoded in the tropical scheme structure. 
Another question arising from the above is the following:

Question 5.1.2. Is it true for tropical cubics that if $\Delta$ vanishes tropically, then we get a repeated tropical root in the generic valuation?

Given a cubic polynomial of the form $x^{3}+A x+B$ with roots $p, q, r=$ $-(p+q)$, we can express the discriminant $\Delta$ in terms of either the roots or the coefficients. We get

$$
\begin{aligned}
\Delta & =-4 A^{3}-27 B^{2} \\
& =4 p^{6}+12 p^{5} q+51 p^{4} q^{2}+82 p^{3} q^{3}+51 p^{2} q^{4}+12 p q^{5}+4 q^{6} .
\end{aligned}
$$

In particular, note that in terms of the roots, the discriminant is a homogeneous polynomial. When we tropicalise $\Delta$ in terms of the roots, therefore, we get the following:

$$
\operatorname{trop}(\Delta)=\min \{6 v(p), 5 v(p)+v(q), \ldots, v(p)+5 v(q), 6 v(q)\} .
$$

Clearly if the valuation of each root is the same, then we will get a tropical root of the generic valuation of $(*)$. It's also clear that in order for any two of the terms in this form of $\operatorname{trop}(\Delta)$ to be equal, then we must have $v(p)=v(q)$.

However, there are certain cases where the valuation of each root is the same, but we do not get a root of the tropical discriminant when it is expressed in terms of the coefficients. This happens when there is cancellation in the coefficients - that is, the valuation of the coefficients deviates from the expected value. The valuations of the roots $p$ and $q$ and the valuations of the coefficients do not determine each other in general.

Example 5.1.3. Consider the cubic given by

$$
x^{3}+\left(\sqrt{3} i t^{4}+t^{6}\right) x+\left(-t^{3}+\sqrt{3} i t^{5}+t^{7}\right),
$$

which has roots $p=\frac{\sqrt{3} i-1}{2} t+t^{3}, q=t$, and $r=-\frac{\sqrt{3} i+1}{2} t-t^{3}$. Clearly we have $v(p)=v(q)=v(r)=1$, and so if we consider the discriminant in terms of the roots, we get

$$
\operatorname{trop}(\Delta)=\{6,6,6,6,6,6,6\},
$$

and clearly the minimum is met at least twice. However, we have $v(A)=4$ and $v(B)=3$, so when we calculate the discriminant in terms of the coefficients, we get

$$
\operatorname{trop}(\Delta)=\min \{12,6\}
$$

and thus the minimum is not achieved twice.

From this we can conclude that, when working in terms of the coefficients, a tropical root of $\operatorname{trop}(\Delta)$ expressed in terms of $A$ and $B$ (i.e., the minimum is achieved at least twice) implies that the valuations of the roots are equal, but the converse is not true. 
5.1.1. Generating the Plücker Coordinates. The underlying linear space of a homogeneous tropical ideal can be represented as the row space of a series of matrices, one for each degree. We construct such a matrix for degree $d$ by making a table with all possible $(d-3)$-degree monomial multiples of $x^{3}+A x z^{2}+B z^{3}$ labelling the rows, and every possible monomial of degree $d$ labelling the columns. We put the coefficient of each monomial in $x^{i} y^{j} z^{k}\left(x^{3}+A x z^{2}+B z^{3}\right)$ in its corresponding column, then rearrange the columns as necessary to make the pattern more clear.

We know from Section 3.10 that the Plücker coordinates completely describe the linear space of an ideal $I$; their valuations determine the tropical linear space of $\operatorname{trop}(I)$.

The first few of these matrices, for degree $3,4,5$ and 6 , are as follows:

$$
\begin{gathered}
{\left[\begin{array}{llll}
1 & 0 & A & B
\end{array}\right]} \\
{\left[\begin{array}{ccccc}
1 & 0 & A & B & 0 \\
0 & 1 & 0 & A & B
\end{array}\right]} \\
{\left[\begin{array}{cccccc}
1 & 0 & A & B & 0 & 0 \\
0 & 1 & 0 & A & B & 0 \\
0 & 0 & 1 & 0 & A & B
\end{array}\right]} \\
{\left[\begin{array}{lllllll}
1 & 0 & A & B & 0 & 0 & 0 \\
0 & 1 & 0 & A & B & 0 & 0 \\
0 & 0 & 1 & 0 & A & B & 0 \\
0 & 0 & 0 & 1 & 0 & A & B
\end{array}\right]}
\end{gathered}
$$

By 5.1 .5 (see below), we can focus on the Plücker coordinates of $\left\langle x^{3}+A x z^{2}+B z^{3}\right\rangle$ arising from the maximal minor with $A$ for every entry down the main diagonal, by dropping the first two and last columns.

$$
\begin{gathered}
{[A]} \\
{\left[\begin{array}{cc}
A & B \\
0 & A
\end{array}\right]} \\
{\left[\begin{array}{ccc}
A & B & 0 \\
0 & A & B \\
1 & 0 & A
\end{array}\right]} \\
{\left[\begin{array}{cccc}
A & B & 0 & 0 \\
0 & A & B & 0 \\
1 & 0 & A & B \\
0 & 1 & 0 & A
\end{array}\right]}
\end{gathered}
$$


The corresponding Plücker coordinates begin as follows

$$
\begin{aligned}
& \gamma_{1}=A \\
& \gamma_{2}=A^{2} \\
& \gamma_{3}=A^{3}+B^{2} \\
& \gamma_{4}=A^{4}+2 A B^{2} \\
& \gamma_{5}=A^{5}+3 A^{2} B^{2} \\
& \gamma_{6}=A^{6}+4 A^{3} B^{2}+B^{4}
\end{aligned}
$$

where $i$ represents the degree $i+2$ piece of the ideal. The minors of a matrix can be easily calculated using Macaulay2, however calculating the first few by hand was the easiest way to find a recurrance relation.

Proposition 5.1.4. The $\gamma_{i}$ are generated by the following recurrence relation:

$$
\gamma_{i+1}=A \gamma_{i}+B^{2} \gamma_{i-2},
$$

where $\gamma_{-1}=0$ and $\gamma_{0}=1$.

Proof. To see this is true, take the following $(i+1) \times(i+1)$ matrix.

$$
\left[\begin{array}{ccccc}
A & B & 0 & \ldots & 0 \\
0 & A & B & \ldots & 0 \\
1 & 0 & A & \ldots & 0 \\
\vdots & & & \ddots & \vdots \\
0 & 0 & 0 & \ldots & A
\end{array}\right]
$$

Expand along the top row to get $A \gamma_{i}-B \ldots$ and then expand along the second row from the top and left-hand column to get

$$
A \gamma_{i}-B(-B)(1) \gamma_{i-2}=A \gamma_{i}+B^{2} \gamma_{i-2}
$$

as required.

Proposition 5.1.5. The Plücker coordinates of the ideal $\left\langle x^{3}+A x z^{2}+\right.$ $\left.B z^{3}\right\rangle$ are determined by taking the maximal minor where each entry in the central diagonal is $A$. The other Plücker coordinates at each degree are monomial multiples of lower degree Plücker coordinates of this form.

In particular, the $\gamma_{i}$ determine all Plücker coordinates, and their valuations $v\left(\gamma_{i}\right)$ determine the valuated Plücker coordinates.

Proof. This is easily verified to be true for small values of $d$, so we will proceed by induction on the degree. Assume that the proposition is true for all $d \leq k$, that is the $k \times k$ matrix below determines all Plücker 
coordinates in degree $k$.

$$
\left[\begin{array}{ccccc}
A & B & 0 & \ldots & 0 \\
0 & A & B & \ldots & 0 \\
1 & 0 & A & \ldots & 0 \\
& \ddots & & & \vdots \\
0 & 0 & 0 & \ldots & A
\end{array}\right]
$$

By hypothesis, we need only take these $k$ columns in degree $k+1$ and check that all possible combinations with additional columns either give us $\gamma_{k+1}$ in the case of the column with $A$ as its top entry, or monomial multiples of lower-degree Plücker coordinates. Given the following $(k+1) \times k$ matrix

$$
\left[\begin{array}{ccccc}
B & 0 & 0 & \ldots & 0 \\
A & B & 0 & \ldots & 0 \\
0 & A & B & \ldots & 0 \\
1 & 0 & A & \ldots & 0 \\
& \ddots & & & \vdots \\
0 & 0 & 0 & \ldots & A
\end{array}\right]
$$

there are four possible additional columns we could add to it:

(1) $(A, 0,1,0, \ldots, 0)^{T}$

(2) $(0,1,0, \ldots, 0,0)^{T}$

(3) $(1,0,0, \ldots 0,0)^{T}$

(4) $(0,0, \ldots, 0,0, B)^{T}$

In case (1) we simply get $\gamma_{k+1}$. In case (2), we get $B \gamma_{k-1}$. Case (3) yields $\gamma_{k}$, while case (4) gives $B^{k+1}$. (It doesn't matter where in the matrix we insert the extra column, since we can always exchange the columns to rearrange it, while preserving the sign). Thus the inductive step is proved.

Proposition 5.1.6. We give a closed form expression for $\gamma_{i}$. We have:

$$
\gamma_{i}=A^{i+1}+\sum_{\{n \in \mathbb{N}: i+1-3 n \geq 0\}} P_{n}(i+1-3 n) A^{i+1-3 n} B^{2 n}
$$

where $P_{n}(j)=\left(\begin{array}{c}j+n-1 \\ n\end{array}\right)$.

Proof. We will proceed by induction on $i$ using the recurrence relation in 5.1.4. The formula above can be rewritten as follows:

$$
\gamma_{i}=A^{i+1}+\sum_{\{n \in \mathbb{N}: i+1-3 n \geq 0\}}\left(\begin{array}{c}
i-2 n \\
n
\end{array}\right) A^{i+1-3 n} B^{2 n} .
$$


Putting this into the recurrence relation $\delta_{i+1}=A \gamma_{i}+B^{2} \gamma_{i-2}$ yields

$$
\begin{aligned}
\gamma_{i+1}= & A\left[A^{i+1}+\sum\left(\begin{array}{c}
i-2 n \\
n
\end{array}\right) A^{i+1-3 n} B^{2 n}\right] \\
& +B^{2}\left[A^{i-1}+\sum\left(\begin{array}{c}
i-2 n-2 \\
n
\end{array}\right) A^{i-1-3 n} B^{2 n}\right] \\
= & A^{i+2}+\sum\left(\begin{array}{c}
i-2 n \\
n
\end{array}\right) A^{i+2-3 n} B^{2 n} \\
& +A^{i-1} B^{2}+\sum\left(\begin{array}{c}
i-2 n-2 \\
n
\end{array}\right) A^{i-1-3 n} B^{2(n+1)} \\
= & A^{i+2}+\left(\begin{array}{c}
i-2 \\
1
\end{array}\right) A^{i-1}+A^{i-1} B^{2} \\
& +\left(\begin{array}{c}
i-4 \\
2
\end{array}\right) A^{i-4} B^{4}+\left(\begin{array}{c}
i-4 \\
1
\end{array}\right) A^{i-4} B^{4} \\
& +\ldots \\
& +\left(\begin{array}{c}
i-2 n \\
n
\end{array}\right) A^{i+2-3 n} B^{2 n}+\left(\begin{array}{c}
i-2 n \\
n-1
\end{array}\right) A^{i+2-3 n} B^{2 n} \\
= & A^{i+2}+\sum\left(\begin{array}{c}
i+1-2 n \\
n
\end{array}\right) A^{i+2-3 n} B^{2 n} \\
= & \gamma_{i+1}
\end{aligned}
$$

Where the final step is justified because

$$
\begin{aligned}
\left(\begin{array}{l}
n \\
k
\end{array}\right)+\left(\begin{array}{c}
n \\
k-1
\end{array}\right) & =\frac{n ! \cdot(n-k+1)}{k !(n+1-k) !}+\frac{n ! \cdot k}{k !(n+1-k) !} \\
& =\frac{(n+1) !}{k !(n+1-k) !}=\left(\begin{array}{c}
n+1 \\
k
\end{array}\right) .
\end{aligned}
$$

Since we know that the first six $\gamma_{i}$ fit this formula, by induction we have shown that all $\gamma_{i}$ can be written explicitly this way.

5.2. Two Tropical Cubics. In this section we examine an example of two cubic curves $x^{3}+A x z^{2}+B z^{3}$ with coefficients in $\mathbb{C}\{\{t\}\}$ with the same tropicalisation, but different valuated discriminants. This prompts us to ask, for a given tropical cubic $x^{3} \oplus v(A) \odot x z^{2} \oplus v(B) \odot z^{3}$, what valuated discriminants are possible?

Consider the following two cubics:

(1) $f_{1}=x^{3}+\left(-3 t^{2}+t^{3}\right) x z^{2}+2 t^{3} z^{3}$

(2) $f_{2}=x^{3}+\left(-3 t^{2}-t^{3}\right) x z^{2}+\left(2 t^{3}+t^{4}\right) z^{3}$

Clearly both of these polynomials have the same set-theoretic tropicalisation, i.e.,

$$
\operatorname{trop}\left(f_{1}\right)=\operatorname{trop}\left(f_{2}\right)=x^{3} \oplus 2 \odot x z^{2} \oplus 3 \odot z^{3},
$$

so they determine the same tropical variety. However, the ideals they generate have potentially distinct tropicalisations. For cubics of the 
form $x^{3}+A x z^{2}+B z^{3}$ we know that the discriminant $\Delta=-4 A^{3}-27 B^{2}$. Tropicalising this gives $\operatorname{trop}(\Delta)=\min (3 v(A), 2 v(B))$. Let us compare the valuation of the discriminants with their generic valuations.

(1) For $f_{1}$ we have

$$
\begin{aligned}
\Delta_{3} & =-\left(4\left(-3 t^{2}+t^{3}\right)+27\left(2 t^{3}\right)^{2}\right) \\
& =-\left(-108 t^{6}+108 t^{7}-36 t^{8}+t^{9}+108 t^{6}\right) \\
& =-108 t^{7}+36 t^{8}-4 t^{9}
\end{aligned}
$$

Valuating this gives $v(\Delta)=7$. On the other hand, using the formula $\operatorname{trop}(\Delta)=\min (3 v(A), 2 v(B))$, we get

$$
\operatorname{trop}(\Delta)(v(A), v(B))=\min (3(2), 2(3))=\min (6,6)=6 \text {. }
$$

Thus due to cancellation, the valuation of the discriminant differs from its generic valuation.

(2) For $f_{2}$ we have

$$
\begin{aligned}
\Delta_{3} & =-\left(4\left(-3 t^{2}-t^{3}\right)+27\left(2 t^{3}+t^{4}\right)^{2}\right) \\
& =-\left(-108 t^{6}-108 t^{7}-36 t^{8}-t^{9}+108 t^{6}+108 t^{7}+27 t^{8}\right) \\
& =-117 t^{8}-4 t^{9}
\end{aligned}
$$

Valuating this gives $v(\Delta)=8$. On the other hand, using the formula $\operatorname{trop}(\Delta)=\min (3 v(A), 2 v(B))$, we get

$$
\operatorname{trop}(\Delta)(v(A), v(B))=\min (3(2), 2(3))=\min (6,6)=6 \text {. }
$$

Again, the valuation discriminant differs from its generic valuation.

As we will see in Section 5.3 below, the roots of $\Delta$ are never roots of the Plücker coordinates of the linear space of the ideal $\left\langle x^{3}+A x z^{2}+B z^{3}\right\rangle_{d}$ for any $d$, thus $\Delta$ cannot be a factor of the Plücker coordinates and no cancellation of lowest terms can occur in the Plücker coordinates. This means the ideals generated by the two cubics must generate the same tropical linear space.

The above example suggests the following:

Conjecture 5.2.1. For any given tropical cubic of the form $T=$ $x^{3} \oplus v(A) \odot x z^{2} \oplus v(B) \odot z^{3}$, we can find infinitely many cubics in $\mathbb{C}\{\{t\}\}[x, z]$ which tropicalise to $T$, and the discriminants $\Delta$ of these cubics have valuations in the range $a \leq \Delta, \Delta \in \mathbb{Q}$, where $a$ is the expected valuation of the discriminant.

Depending on the coefficients and powers in the $A, B \in \mathbb{C}\{\{t\}\}$, if the first two terms of $A$ and $B$ cancel each other out, then the second terms may also cancel, and third, and so on, which would allow for any valuation of the discriminant higher than the expected value $a$. 
5.3. Proof That $v\left(\Delta_{3}\right)$ Cannot be Recovered from $\operatorname{trop}\left(\left\langle x^{3}+\right.\right.$ $\left.\left.A x z^{2}+B z^{3}\right\rangle\right)$. In this section, we prove the following result:

Theorem 5.3.1. The discriminant $\Delta=-\left(4 A^{3}+27 B^{2}\right)$ of the cubic $x^{3}+A x z^{2}+B z^{3}$ cannot appear as a factor in the Plücker coordinates of the ideal $\left\langle x^{3}+A x z^{2}+B z^{3}\right\rangle$.

The valuation of the discriminant is not in general determined by the valuations of the Plucker coordinates of the ideal; if all Plucker coordinates are equal to their generic valuations then the valuation of the discriminant may or may not be equal to its generic valuation. However, we have:

Corollary 5.3.2. If one of the valuated Plücker coordinates deviates from its generic valuation then the valuation of the discriminant is equal to its generic valuation, which is determined by the degree 3 part of the tropical ideal.

Proof. Suppose we have a cubic $x^{3}+A x+B$, with roots $p, q, r$. Then

$$
\begin{aligned}
x^{3}+A x+B & =(x-p)(x-q)(x-r) \\
& =x^{3}-(p+q+r) x^{2}+(p q+p r+q r) x-p q r .
\end{aligned}
$$

Since the coefficient of $x^{2}$ is zero, we get $p=-(q+r)$, and substituting this into the other coefficients gives

$$
\begin{aligned}
& A=-\left(q^{2}+q r+r^{2}\right) \\
& B=q^{2} r+q r^{2} .
\end{aligned}
$$

Put $u=q^{2}+q r$, so that we get

$$
\begin{aligned}
& A=-\left(u+r^{2}\right) \\
& B=u r .
\end{aligned}
$$

The recurrence relation becomes

$$
\gamma_{i+1}=-\left(u+r^{2}\right) \gamma_{i}+u^{2} r^{2} \gamma_{i-2},
$$

and the discriminant $\Delta=-4 A^{3}-27 B^{2}$ becomes

$$
4 r^{6}+12 u r^{4}-15 u^{2} r^{2}+4 u^{3},
$$

from which we find that $\Delta$ has roots at $u=\frac{-r^{2}}{4}, u=2 r^{2}$ and $u=2 r^{2}$.

To show that the tropicalisations of the previous examples are the same, we need to show that cancellation in the discriminant affecting its valuation does not affect the valuations of the $\gamma_{i}$. In order to show this, we will show that the discriminant $\Delta$ is never a factor of the $\gamma_{i}$.

Since the discriminant $\Delta=\left(4 u+r^{2}\right)\left(u-2 r^{2}\right)^{2}$ and the $\gamma_{i}$ are homogeneous polynomials in $u$ and $r^{2}$ with integer coefficients, we know that $\Delta$ is a factor if $\gamma_{i}$ only if $\frac{-r^{2}}{4}$ and $2 r^{2}$ are roots of the $\gamma_{i}$. In what follows, we will show that $2 r^{2}$ and $\frac{-r^{2}}{4}$ is never a root of $\gamma_{i}$ for any value of $i$, and therefore $\Delta$ does not appear as a factor in $\gamma_{i}$ for any value of $i$. 
Suppose $u=2 r^{2}$. Then the recurrence relation gives us

$$
\gamma_{i+1}=-3 r^{2} \gamma_{i}+4 r^{6} \gamma_{i-2}
$$

The sequence of $\gamma_{i}$ begins as follows:

$$
\begin{aligned}
\gamma_{0} & =-3 r^{2} \\
\gamma_{1} & =9 r^{4} \\
\gamma_{2} & =-23 r^{6} \\
\gamma_{3} & =57 r^{8}
\end{aligned}
$$

It would seem that the $\gamma_{i}$ is a constant times some power of $r$. We will prove by induction that this is true for all values of $i$.

Suppose that for some natural number $k$ and some constants $a_{k}, a_{k-2}$, $\gamma_{k}=a_{k} r^{2(k+1)}$ and $\gamma_{k-2}=a_{k-2} r^{2(k-1)}$. Then the recurrance relation gives us

$$
\begin{aligned}
\gamma_{k+1} & =-3 r^{2} \gamma_{k}+4 r^{6} \gamma_{k-2} \\
& =-3 r^{2} a_{k} r^{2(k+1)}+4 r^{6} a_{k-2} r^{2(k-1)} \\
& =-3 a_{k} r^{2 k+4}+4 a_{k-2} r^{2 k+4} \\
& =\left(-3 a_{k}+4 a_{k-2}\right) r^{2 k+4}
\end{aligned}
$$

Since the hypothesis is clearly true for $\gamma_{0}, \gamma_{1}$ and $\gamma_{2}$, we can conclude by induction that this is true for all values of $i$.

From the above, we get that the $a_{k}$ are determined by the recurrence relation

$$
a_{k+1}=-3 a_{k}+4 a_{k-2} .
$$

From this, we can work out that in general $a_{k}$ is given by the following formula:

$$
a_{k}=\frac{1}{9}\left[1+(8+6 k)(-2)^{k}\right]
$$

For any integer $k>0$, clearly $a_{k} \neq 0$. Thus we have shown that if $\gamma_{k}=a_{k} r^{2(k+1)}$ for all $k$, then the only roots are at $r=0$.

Now suppose that $u=\frac{r^{2}}{4}$. The recurrence relation for the $\gamma_{i}$ becomes

$$
\gamma_{i+1}=-\frac{3 r^{2}}{4} \gamma_{i}+\frac{r^{6}}{4} \gamma_{i-2}
$$

and we get that

$$
\gamma_{k}=\frac{a_{k}}{4^{k}} r^{2(k+1)}
$$

where $a_{k}$ is as above. Thus in this case also, the Plücker coordinates are zero only if $r=0$.

This implies in turn that the only time the discriminant of a cubic shares roots with the Plücker coordinates is when the roots are all zero, i.e., when the coefficients $A$ and $B$ in $x^{3}+A x z^{2}+B z^{3}$ are zero. 
Thus, cancellation in the Plücker coordinates and cancellation in the discriminant are mutually exclusive possibilities.

5.4. Polynomials of the form $x^{n}+A x z^{n-1}+B z^{n}$. We can generalise our results on the Plücker coordinates of $\left\langle x^{3}+A x z^{2}+B z^{3}\right\rangle$ to the Plücker coordinates of $\left\langle x^{n}+A x z^{n-1}+B z^{n}\right\rangle$ with the following result and a lemma which can be proved using the trinomial formula for discriminants.

Proposition 5.4.1. The Plücker coordinates $\alpha_{i}$ obtained from the diagonal of the Macaulay matrix for which every entry is A are determined by the recurrence relation

$$
\alpha_{i+1}=A \alpha_{i}+(-1)^{n-1} B^{n-1} \alpha_{i-(n-1)} .
$$

Furthermore, all Plücker coordinates are determined by this recurrence relation up to multiplication by a monomial.

Lemma 5.4.2. The discriminant $\Delta$ of the polynomial $x^{n}+A x z^{n-1}+B z^{n}$ is given, up to sign, by the formula

$$
\Delta=n^{n} B^{n-1}+(-1)^{n}(n-1)^{n-1} A^{n} .
$$

In particular, this means that if $\Delta=0$, then

$$
B^{n-1}=(-1)^{n-1} \frac{(n-1)^{n-1}}{n^{n}} A^{n} .
$$

Proof. We use the trinomial formula given as (1.38) in Chapter 12 of [7], which is valid for trinomials of the form $A x^{n}+B x^{m}+C$, where $0<m<n$ and $m$ and $n$ are relatively prime. Since $n$ and 1 are always relatively prime, the formula is valid.

We have seen in previous sections that Lemma 5.4.2 is true for $n=3$, and is also true when $n=4,5$, when we get $\Delta=256 B^{3}-27 A^{4}$ and $\Delta=3125 B^{4}+256 A^{5}$ respectively (by calculating directly from the resultant of the polynomial and its derivative). We can use a similar method to that in 5.3 to show that the vanishing loci of the discriminant and Plücker coordinates cannot coincide.

Proof of 5.4.1. Take the $k \times k$ submatrix of the Macaulay matrix for degree $n-1+k$ for which each entry along the leading diagonal is $A$. Its determinant can be found by expanding down the first column. We get $A \cdot \alpha_{k-1}+(-1)^{n-1} \ldots$, then we repeatedly expand along the top row to get $A \alpha_{k-1}+(-1)^{n-1} B^{n-1} \alpha_{k-(n-1)}$ as required.

We will show the second part of the proposition by modifying the proof of 5.1.5. Cases (1), (3) and (4) from that proof are exactly the same here, so we need only consider the case (2), where we have a column where every entry is zero except for the $j^{\text {th }}$ entry $(2 \leq j \leq n-1)$. Expanding along that column gives us $(-1)^{j-1} B^{j-1} \alpha_{k+1-(j-1)}$. Thus in this case also, the Plücker coordinates are determined, up to multiplication by a monomial, completely by the recurrence relation given above. 
Now, to show that the vanishing loci of the Plücker coordinates and discriminant cannot coincide, we put $B^{n-1}=(-1)^{n-1} \frac{(n-1)^{n-1}}{n^{n}} A^{n}$ into the recurrence relation to get

$$
\alpha_{i+1}=A \alpha_{i}+\frac{(n-1)^{n-1}}{n^{n}} A^{n} \alpha_{i-(n-1)} .
$$

For $1 \leq i \leq n-1$, we have $\alpha_{i}=A^{i}$. Thus we get that

$$
\begin{aligned}
\alpha_{n} & =A \alpha_{n-1}+\frac{(n-1)^{n-1}}{n^{n}} A^{n} \alpha_{0} \\
& =A \cdot A^{n-1}+\frac{(n-1)^{n-1}}{n^{n}} A^{n} \cdot 1 \\
& =\left(1+\frac{(n-1)^{n-1}}{n^{n}}\right) A^{n}
\end{aligned}
$$

This pattern continues to give

$$
\alpha_{k}=\left(1+(k-n) \frac{(n-1)^{n-1}}{n^{n}} A^{n}\right) A^{k},
$$

which does not vanish unless $A=0$.

Thus we have proved the following:

Theorem 5.4.3. The valuation of the discriminant of a polynomial of the form $x^{n}+A x z^{n-1}+B z^{n}$ is not determined by the valuations of the Plücker coordinates of the ideal $\left\langle x^{n}+A x z^{n-1}+B z^{n}\right\rangle$.

It is worth noting that with the aid of Propositon 4.4.6, we can in fact generalise this result to ideals determined by polynomials of the form $x^{m n}+A x^{m} z^{m(n-1)}+B z^{m n}$.

5.5. The Plücker Coodinates of $\left\langle x^{3}+A x z^{2}+B z^{3}\right\rangle$ and $\Delta$ in Fields of Characteristic $p$. Another avenue of interest is what happens when we reduce the Plücker coordinates modulo $p$ for some prime integer. We first consider the trivial cases where $p=2,3$ in 5.5.1, and then look at larger primes. We will look at the small primes $p=5,7,11,13,17,19,23$ in 5.5 .2 .

5.5.1. Characteristic 2 and 3. Reducing the Plücker coordinates and discriminant mod 2 and 3 is not particularly interesting, due to the coefficients in the two terms of the discriminant $-4 A^{3}-27 B^{2}$. This will not be useful for studying the $j$-invariant, but we make a note of it regardless. In the mod 2 case the discriminant is a monomial in $B$ (we have $\Delta=B^{2}$ ), and in the mod 3 case the discriminant is a monomial in $A$ (we have $\Delta=2 A^{3}$ ). What this means is that when we're working with cubics of this form with coefficients in $\mathbb{F}_{p}\{\{t\}\}$ for $p=2$, 3, it isn't possible to get cancellation in the discriminant, and therefore it isn't possible to have two cubics with the same set-theoretic tropicalisation and different discriminants as it is in characteristic 0 . This should carry 
through to cubics with coefficients in $\mathbb{F}_{2^{n}}\{\{t\}\}$ and $\mathbb{F}_{3^{n}}\{\{t\}\}$ and also their algebraic closures

$$
\overline{\mathbb{F}_{p}}\{\{t\}\}=\cup_{n \in \mathbb{N}} \mathbb{F}_{p^{n}}\{\{t\}\},
$$

or any other field extension, for $p=2,3$.

5.5.2. Characteristic $p$ for $p<27$. First we will look at $p=5$, which yields an interesting result quite quickly. We will first look at cubics of the form $x^{3}+A x z^{2}+B x^{3}$ where $A, B \in \mathbb{F}_{5}\{\{t\}\}$, then consider how this applies to the algebraic closure $\overline{\mathbb{F}_{5}}\{\{t\}\}$ or any other field extension of $\mathbb{F}_{p}$.

Reducing the discriminant modulo 5 yields $\Delta=A^{3}+3 B^{2}$. Recall that $\delta_{5}=A^{5}+3 A^{2} B^{2}=A^{2} \Delta$. Thus we can clearly see that the Plücker coordinates are not independent of the discriminant. This means that there is cancellation affecting the valuation of $\delta_{5}$ if and only if there is cancellation affecting the valuation of the discriminant, and therefore it's not possible to have two cubics with the same scheme-theoretic tropicalisation and different valuated discriminants. We will illustrate this with an example where $A, B \in \mathbb{F}_{5^{2}}$.

Example 5.5.1. Let $\lambda^{2}=2$, so that $\mathbb{F}_{25}=\left\{u+w \lambda: u, w \in \mathbb{F}_{5}\right\}$. Let

(1) $p_{1}(x, z)=x^{3}+t^{2} x z^{2}+\left(2 \lambda t^{3}+3 t^{4}\right) z^{3}$, and

(2) $p_{2}(x, z)=x^{3}+t^{2} x z^{2}+t^{3} z^{3}$.

We have that $\operatorname{trop}\left(p_{1}\right)=\operatorname{trop}\left(p_{2}\right)=\min \{3 x, 2+x+2 z, 3+3 z\}$, but if we look at the discriminants, we get

(1) $\Delta\left(p_{1}\right)=t^{6}+4 t^{6}+\lambda t^{7}+2 t^{8}=\lambda t^{7}+2 t^{8}$, and

(2) $\Delta\left(p_{2}\right)=t^{6}+3 t^{6}=4 t^{6}$.

The valuation of (1) is 7 whereas the valuation of (2) is 6 . In the characteristic 0 case, we wouldn't be able to detect this in the Plücker coordinates, however here we can look at $\delta_{5}$. We have

(1) $\delta_{5}\left(p_{1}\right)=t^{4}\left(\lambda t^{7}+2 t^{8}\right)$, and

(2) $\delta_{5}\left(p_{2}\right)=4 t^{10}$.

These clearly have different valuations, 11 and 10 respectively. Thus the valuated Plücker coordinates can detect cancellation in $\Delta$ over $\mathbb{F}_{25}\{\{t\}\}$.

Because the discriminant $\Delta=A^{3}+3 B^{2}$ appears as a factor in $\delta_{5}$ when $A, B \in \mathbb{F}_{5}$, it will appear as a factor of $\delta_{5}$ when $A, B$ are in any extension of that field. Since the algebraic closure of $\mathbb{F}_{5}$ is an infinite union of such extensions, we see that the result proved in 5.3 for $A, B \in \mathbb{C}\{\{t\}\}$ is false for $A, B \in \overline{\mathbb{F}_{5}}\{\{t\}\}$.

The case where $p=23$ is equally straightforward. In that case the discriminant $\Delta=19\left(A^{3}+B^{2}\right)$, and $\delta_{3}=A^{3}+B^{2}$, implying that the Plücker coordinates are not independent of the discriminant for cubics with coefficients in $\overline{\mathbb{F}_{23}}\{\{t\}\}$. 
The cases $p=7,11,13,17,19$ are somewhat less straightforward. We have to look at the factors of the Plücker coordinates to find the discriminant.

When $p=7$, we have

$$
\Delta=3\left(A^{3}+5 B^{2}\right)
$$

and

$$
\delta_{21}=\left(A^{3}+5 B^{2}\right)\left(A^{18}+3 A^{12} B^{2}+6 A^{9} B^{4}+6 A^{6} B^{6}+5 A^{3} B^{8}+3 B^{10}\right) .
$$

When $p=11$, we have

$$
\Delta=7\left(A^{3}+4 B^{2}\right),
$$

and

$\delta_{22}=A\left(A^{3}+4 B^{2}\right)\left(A^{18}+5 A^{15}+A^{12} B^{4}+6 A^{9} B^{6}+9 A^{6} B^{8}+8 A^{3} B^{10}+2 B^{12}\right)$.

When $p=13$, we have

$$
\Delta=9\left(A^{3}+10 B^{2}\right),
$$

and

$$
\begin{aligned}
\delta_{24}= & \left(A^{3}+10 B^{2}\right)\left(A^{3}+4 B^{2}\right)\left(A^{3}+11 B^{2}\right) \\
& \cdot\left(A^{15}+10 A^{12} B^{2}+6 A^{9} B^{4}+2 A^{6} B^{6}+2 A^{3} B^{8}+6 B^{10}\right) .
\end{aligned}
$$

When $p=17$, we have

$$
\Delta=12\left(A^{3}+11 B^{2}\right) .
$$

For $i \leq 25$, none of the $\delta_{i}$ factorise in such a way as to include $\Delta$ as a factor; see question below.

When $p=19$, we have

$$
\Delta=15\left(A^{3}+2 B^{2}\right)
$$

and

$\delta_{20}=A^{2}\left(A^{3}+2 B^{2}\right)\left(A^{3}+15 B^{2}\right)\left(A^{12}+A^{9} B^{2}+16 A^{6} B^{4}+5 A^{3} B^{6}+6 B^{8}\right)$.

We have therefore proved the following:

Proposition 5.5.2. The Plücker coordinates of cubics of the form $x^{3}+A x z^{2}+B z^{3}$ are not independent of the discriminant when $A, B \in$ $\overline{\mathbb{F}_{p}}\{\{t\}\}$, for $p=5,7,11,13,19,23$.

Using this, we ask the following question.

Question 5.5.3. For some values of $p$, the Plücker coordinates of ideals of the form $\left\langle x^{3}+A x z^{2}+B z^{3}\right\rangle$ are not independent of the discriminant when $A, B \in \mathbb{K}$ for some field $\mathbb{K}$ of characteristic $p$. For which values of $p$ is this true? 
For larger values of $p$, we might be able to use similar techniques to find Plücker coordinates with the discriminant as a factor. When $p=29$, we have $\Delta=2\left(A^{3}+22 B^{2}\right)$ and when $p=31$, we have $\Delta=4\left(A^{3}+30 B^{2}\right)$ - there is no reason why the Plücker coordinates might not factorise in a similar way, but no evidence yet that they do either.

5.6. The Plücker Coordinates of $\left\langle y^{2} z-x^{3}-A x z^{2}-B z^{3}\right\rangle$. In this section we will briefly explain how some of our findings can be applied to tropical elliptic curves. We will focus on elliptic curves over fields of characteristic zero here, and Section 5.7 below will give some results for elliptic curves over fields of characteristic $p$.

First note that if we have a cubic in Weierstrass form given by $f(x, z)=x^{3}+A x z^{2}+B z^{3}$, then the corresponding elliptic curve is given by $y^{2} z-f(x, z)$, so we can modify Proposition 4.2 .2 to get that the Macaulay matrix for the ideal $\left\langle y^{2} z-f(x, z)\right\rangle$ has the following form, where $M_{i}$ is the Macaulay matrix of the degree $i-n+1$ part of the ideal $\langle f(x, z)\rangle$ and $J_{i}$ isthe rectangular matrix consisting of all zeroes, except the rightmost $i$ columns, which are the $i \times i$ identity matrix.

$$
\left[\begin{array}{ccccccccc}
-M_{d} & 0 & J_{d} & 0 & \cdots & 0 & 0 & 0 & 0 \\
0 & -M_{d-1} & 0 & J_{d-1} & \cdots & 0 & 0 & 0 & 0 \\
& & \ddots & & \ddots & & & & \\
0 & & \cdots & & & J_{4} & 0 & 0 & 0 \\
0 & & \cdots & & \ddots & 0 & J_{3} & 0 & 0 \\
0 & & \cdots & & \cdots & -M_{2} & 0 & J_{2} & 0 \\
0 & & \cdots & & \cdots & 0 & -M_{1} & 0 & J_{1}
\end{array}\right]
$$

From this we can see that, of the non-zero Plücker coordinates, some of them are straightforward products of Plücker coordinates of the ideal $\langle f(x, z)\rangle$, in which case we have proved that their valuations are independent of the valuation of the discriminant. Recall that the formula for the $j$-invariant is given by

$$
j=1728 \frac{4 A^{3}}{-\Delta}
$$

thus the valuation of the $j$-invariant depends on the valuation of $A$ and of the discriminant $\Delta$. We therefore have the following partial result:

Proposition 5.6.1. The valuation of the $j$-invariant of the elliptic curve given by $y^{2} z-x^{3}-A x z^{2}-B z^{3}$ is independent of the valuations of the subset of Plücker coordinates consisting of products of the form $\prod_{j} \delta_{j}$, where the $\delta_{j}$ are Plücker coordinates of the ideal $\left\langle x^{3}+A x z^{2}+B z^{3}\right\rangle$.

However, recall from Section 4.3 that in the case of a quadratic $f(x, z)=x^{2}+B x z+C z^{2}$, the valuations of the Plücker coordinates of the ideal $\left\langle y^{2}+f(x, z)\right\rangle$ are affected by the valuation of the discriminant 
$\Delta_{2}=B^{2}-4 C$. Thus it seems reasonable to ask if the same is also true in this case.

Question 5.6.2. Does the valuation of the $j$-invariant depend on the valuations of the Plücker coordinates not covered in 5.6.1? Is there a neat way to describe these other Plücker coordinates?

\subsection{The Plücker Coordinates of Tropical Elliptic Curves in}

Characteristic $p$. We will begin by making some observations about some special cases of elliptic curves in characteristic 2 and 3, before looking at fields of characteristic $p \geq 5$.

5.7.1. Characteristic 2 and 3. Recall that in characteristic 2, there are two possible forms for an equation of an elliptic curve:

(1) $y^{2}+x y=x^{3}+C x+D$

(2) $y^{2}+E y=x^{3}+C x+D$

In case $(1)$, the elliptic curve has $j$-invariant $\frac{1}{D}$, in which case its valuation is entirely dependent on the valuation of the degree 3 Plücker coordinate $D$. In case (2), the elliptic curve has $j$-invariant 0 , and always has valuation $\infty$ regardless of the valuations of the Plücker coordinates.

Now suppose that we have an elliptic curve over a field of characteristic 3 with equation of one of the following forms:

(1) $y^{2}=x^{3}+B x^{2}+D$

(2) $y^{2}=x^{3}+C x+D$

In case (1), the formula for the $j$-invariant becomes $\frac{-B^{3}}{D}$, the valuation of which depends only on the valuations of the degree 3 Plücker coordinates $B$ and $D$. In case (2), the $j$-invariant is always 0 with valuation $\infty$, regardless of the valuations of the Plücker coordinates.

In these cases, the question of whether the valuations of the Plücker coordinates determine the valuation of the $j$-invariant has a very straightforward answer.

5.7.2. Characteristic $p \geq 5$. As we saw in Section 5.5, for most small values of $p$ the discriminant of a cubic appears as a factor in the Plücker coordinates and therefore is not independent of them. Since we know that the highest degree Plücker coordinates of $\left\langle y^{2} z-x^{3}-A x z^{2}-B z^{3}\right\rangle$ in degree $d$ are the product of the first $d$ highest degree Plücker coordinates of $\left\langle x^{3}+A x z^{2}+B z^{3}\right\rangle$ then the discriminant of the cubic $x^{3}+A x z^{2}+B z^{3}$ will appear as a factor in those Plücker coordinates, and thus the $j$-invariant cannot be independent of the Plücker coordinates.

Example 5.7.1. In the vein of Example 5.5.1, we consider the ideals generated by the defining polynomials of the following two elliptic curves. Let $\lambda^{2}=2$, so that $\mathbb{F}_{25}=\left\{u+w \lambda: u, w \in \mathbb{F}_{5}\right\}$. Let

(1) $\mathcal{E}_{1}=y^{2} z-p_{1}(x, z)=y^{2} z-x^{3}-t^{2} x z^{2}-\left(2 \lambda t^{3}+3 t^{4}\right) z^{3}$, and

(2) $\mathcal{E}_{2}=y^{2} z-p_{2}(x, z)=y^{2} z-x^{3}-t^{2} x z^{2}-t^{3} z^{3}$. 
We have that $\operatorname{trop}\left(\mathcal{E}_{1}\right)=\operatorname{trop}\left(\mathcal{E}_{2}\right)=\min \{2 y+z, 3 x, 2+x+2 z, 3+3 z\}$, but if we look at their $j$-invariants, we get

(1) $j\left(\mathcal{E}_{1}\right)=\frac{2 t^{2}}{t^{6}+4 t^{6}+\lambda t^{7}+2 t^{8}}=\frac{2 t^{2}}{\lambda t^{7}+2 t^{8}}$, and

(2) $j\left(\mathcal{E}_{2}\right)=\frac{2 t^{2}}{t^{6}+3 t^{6}}=\frac{2 t^{2}}{4 t^{6}}=3 t^{-4}$.

The valuation of (1) is -5 whereas the valuation of $(2)$ is -4 . In the characteristic 0 case, we don't know if this would affect the valuated Plücker coordinates, however here we can look at the Plücker coordinate $D_{4}$ given by

$$
\prod_{0 \leq i \leq 5} \delta_{i}=-A \cdot A^{2} \cdot\left(A^{3}+B^{2}\right) \cdot\left(A^{4}+2 A B^{2}\right) \cdot\left(A^{5}+3 A^{2} B^{2}\right) .
$$

(1) $D_{4}\left(\mathcal{E}_{1}\right)=2 \lambda t^{31}+t^{32}+4 t^{34}+t^{36}$, and

(2) $D_{4}\left(\mathcal{E}_{2}\right)=4 t^{30}$

These clearly have different valuations, 31 and 30 respectively. Thus the valuated Plücker coordinates can detect cancellation in the $j$-invariant over $\mathbb{F}_{25}\{\{t\}\}$.

More concretely, we have the following result:

Theorem 5.7.2. Let $c$ be the cubic $x^{3}+A x z^{2}+B z^{3}, \mathcal{E}_{c}$ be the elliptic curve defined by the polynomial $y^{2} z-c=y^{2} z-x^{3}-A x z^{2}-B z^{3}$, and let $I$ be the ideal generated by this polynomial. For $p=5,7,11,13,19,23$, the Plücker coordinates of $I$ are not independent of the discriminant of $c$, and therefore the valuation of the $j$-invariant of $\mathcal{E}_{c}$ is not independent of the tropical linear space of trop $(I)$.

5.8. Cubics of the Form $x^{3}+B x^{2} z+D z^{3}$. In this section we take a brief look at the Plücker coordinates of $\left\langle x^{3}+B x^{2} z+D z^{3}\right\rangle$, and show that not all hypersurfaces determined by low-degree polynomials have Plücker coordinates that can be described as simply as in the previous cases we have looked at.

As before, we can take the "central" maximal minors of the Macaulay matrices in each degree and obtain a recurrence relation which determines some of the Plücker coordinates. Expanding the matrix below along the top row, then the columns from the left gives us the recurrence relation $\beta_{k+1}=B \beta_{k}+D \beta_{k-2}$.

$$
\left[\begin{array}{cccccccc}
B & 0 & D & 0 & 0 & \ldots & 0 & 0 \\
1 & B & 0 & D & 0 & \ldots & 0 & 0 \\
0 & 1 & B & 0 & D & \ldots & 0 & 0 \\
0 & 0 & 1 & B & 0 & \ldots & 0 & 0 \\
& & & \ddots & & & \vdots & \\
0 & 0 & 0 & 0 & 0 & \ldots & 0 & B
\end{array}\right]
$$

This gives us the following Plücker coordinates, up to degree 9 (where $\beta_{i}$ is the Plücker coordinate for degree $\left.3+(i-1)\right)$. 


$$
\begin{aligned}
& \beta_{1}=B \\
& \beta_{2}=B^{2} \\
& \beta_{3}=B^{3}+D \\
& \beta_{4}=B^{4}+2 B D \\
& \beta_{5}=B^{5}+3 B^{2} D \\
& \beta_{6}=B^{6}+4 B^{3} D+D^{2}
\end{aligned}
$$

Now consider the following submatrix of the degree 8 Macaulay matrix:

$$
\left[\begin{array}{cccccc}
0 & D & 0 & 0 & 0 & 0 \\
B & 0 & D & 0 & 0 & 0 \\
1 & B & 0 & D & 0 & 0 \\
0 & 1 & B & 0 & D & 0 \\
0 & 0 & 1 & B & 0 & D \\
0 & 0 & 0 & 1 & B & 0
\end{array}\right]
$$

It has determinant $B^{3} D^{3}-D^{4}$, which is not a monomial multiple of any of the Plücker coordinates determined by the recurrence relation. This shows that, except for certain specific cases of polynomials (even those of low degree or trinomials), proving that the valuations of the Plücker coordinates of the ideal they generate are independent of the valuation of their discriminant may not be such an easy task.

5.9. The General Cubic $A x^{3}+B x^{2} z+C x z^{2}+D z^{3}$. In this section we will take a brief look at the more general cubic, determined by the polynomial $A x^{3}+B x^{2} z+C x z^{2}+D z^{3}$. Section 5.8 above tells us that the Plücker coordinates of cubics of this form cannot be so easily described as those of cubics given by polynomials of the form $x^{3}+A x z^{2}+B z^{3}$, but we will include this anyway for completeness.

First of all, we have the recurrence relation for the central Plücker coordinates in each degree (by which we mean the Plücker coordinates with the most terms, resulting from taking the minors consisting of the central columns of the Macaulay matrix). In fact, there are two, and one is obtained from the other via the map that transposes $A$ and $D$, and $B$ and $C$.

Proposition 5.9.1. The recurrence relation for the central Plücker coordinates in each degree of the ideal generated by the cubic $A x^{3}+$ $B x^{2} z+C x z^{2}+D z^{3}$ is given by the recurrence relation

$$
\beta_{n+1}=B \beta_{n}-A C \beta_{n-1}+A^{2} D \beta_{n-2},
$$

or alternatively

$$
\gamma_{n+1}=C \gamma_{n}-B D \gamma_{n-1}+A D^{2} \gamma_{n-2}
$$


Proof. Consider the following submatrix of the Macaulay matrix in some degree $d$ :

$$
\left[\begin{array}{ccccccc}
B & C & D & 0 & 0 & \ldots & 0 \\
A & B & C & D & 0 & \ldots & 0 \\
0 & A & B & C & D & \ldots & 0 \\
0 & 0 & A & B & C & \ldots & 0 \\
0 & 0 & 0 & A & B & \ldots & 0 \\
\vdots & & & & & \ddots & \\
0 & & \ldots & & 0 & \ldots & B
\end{array}\right]
$$

Expand along the far left column first, to get $\beta_{d}=B \beta_{d-1}-A \ldots$, and then expand along the second to left column to get $\beta_{d}=B \beta_{d-1}-$ $A\left(C \beta_{d-2}-A \ldots\right)$. Finally, expand along the top row to give

$$
\begin{aligned}
\beta_{d} & =B \beta_{d-1}-A\left(C \beta_{d-2}-A D \beta_{d-3}\right) \\
& =B \beta_{d-1}-A C \beta_{d-2}+A^{2} D \beta_{d-3},
\end{aligned}
$$

as required. Alternatively, consider the following submatrix of the Macaulay matrix in degree $d$ :

$$
\left[\begin{array}{ccccccc}
C & D & 0 & 0 & 0 & \ldots & 0 \\
B & C & D & 0 & 0 & \ldots & 0 \\
A & B & C & D & 0 & \ldots & 0 \\
0 & A & B & C & D & \ldots & 0 \\
0 & 0 & A & B & C & \ldots & 0 \\
\vdots & & & & & \ddots & \\
0 & & \ldots & & 0 & \ldots & C
\end{array}\right]
$$

Transpose the matrix (preserving the determinant), then follow the procedure above to get the result.

We can easily derive the recurrence relation for the Plücker coordinates of the ideal $\left\langle x^{3}+C x z^{2}+D z^{3}\right\rangle$ by setting $B=0$.

We must note here that it is not true that all Plücker coordinates are determined by those on the two central diagonals, which limits what we can do with just those recurrence relations. It would be useful to know if there is a neat way to express all of the Plücker coordinates of a general cubic.

Using the recurrence relation above, we get that the first few Plücker coordinates $\beta_{d}$, where $3+d$ is the homogeneous degree of the piece of 
the ideal, are as follows:

$$
\begin{aligned}
& \beta_{0}=B \\
& \beta_{1}=B^{2}-A C \\
& \beta_{2}=B^{3}-2 A C B+A^{2} D \\
& \beta_{3}=B^{4}-3 A C B^{2}+2 A^{2} D B+A^{2} C^{2} \\
& \beta_{4}=B^{5}-4 A C B^{3}+3 A^{2} D B^{2}+3 A^{2} C^{2} B-2 A C A^{2} D
\end{aligned}
$$

Now, suppose we scale the polynomial so that $b=1$, and write $x=\frac{a c}{b^{2}}$ and $y=\frac{a^{2} d}{b^{3}}$. We can then rewrite the first few $\beta_{i}$ as follows:

$$
\begin{aligned}
& \beta_{0}=1 \\
& \beta_{1}=1-x \\
& \beta_{2}=1-2 x+y \\
& \beta_{3}=1-3 x+2 y+x^{2} \\
& \beta_{4}=1-4 x+3 y+3 x^{2}-2 x y \\
& \beta_{6}=1-5 x+4 y+6 x^{2}-6 x y+y^{2}-x^{3} \\
& \beta_{7}=1-6 x+5 y+10 x^{2}-12 x y+3 y^{2}-4 x^{3}+3 x^{2} y
\end{aligned}
$$

Note the obvious fact that if we have $d=0$ so that $y=0$ also, the $\beta_{i}$ form the same pattern as the Plücker coordinates in the quadratic case in 4.1. Alternatively, transposing $A$ and $D$, and $B$ and $C$, then setting $C=0$ so that $x=0$ gives us the same pattern as the Plücker coordinates of the cubic in Weierstrass form.

Question 5.9.2. Can the valuation of the discriminant

$$
\Delta_{3}=B^{2} C^{2}-4 A C^{3}-4 B^{3} D-27 A^{2} D^{2}+18 A B C D
$$

of the cubic equation $A x^{3}+B x^{2} z+C x z^{2}+D z^{3}$ be recovered from the Plücker coordinates of the associated ideal? Is there cancellation in the Plücker coordinates when there is cancellation in the discriminant? Does the tropical ideal determine the valuation of the discriminant?

Below is an example describing three monic cubics, each with distinct roots, the first two with cancellation in their discriminant and the third without.

Example 5.9.3. Consider the following tropical cubics:

(1) $p_{1}(x)=x^{3}+\left(t+t^{2}\right) x^{2}-t^{2} x-\left(t^{3}+t^{4}\right)$

(2) $p_{2}(x)=x^{3}+\left(2 t-t^{3}\right) x^{2}+\left(t^{2}-t^{4}\right) x-\left(t^{4}+t^{5}\right)$

(3) $p_{3}(x)=x^{3}+t^{2} x^{2}-t^{2} x-t^{4}$

The roots of $p_{1}(x)$ are $\pm t$ and $t+t^{2}$, all with valuation 1 , and the roots of $p_{2}(x)$ are $t, t+t^{2}$, and $-t^{2}$; the first two having valuation 1 , and 
the latter having valuation 2 . The roots of $p_{3}(x)$ are $\pm t$ and $t^{2}$, with respective valuations 1,1 , and 2 . Let's consider their discriminants below. The discriminant of a monic cubic is given by $\Delta_{3}=B^{2} C^{2}-$ $4 C^{3}-4 B^{3} D-27 D^{2}+18 B C D$; this formula tropicalises to give

$\min \{2 v(B)+2 v(D), 3 v(C), 3 v(B)+v(D), 2 v(D), v(B)+v(C)+v(D)\}$.

(1) We have $\Delta\left(p_{1}\right)=16 t^{8}+16 t^{9}+4 t^{10}$, the valuation of which is 8. Putting the valuations of the coefficients into the tropical formula, however, yields $\min \{6,6,6,6,6\}=6$.

(2) On the other hand, $\Delta\left(p_{2}\right)=-4 t^{7}-31 t^{8}-48 t^{9}-20 t^{10}+6 t^{11}+$ $4 t^{12}-4 t^{13}-3 t^{14}$, the valuation of which is 7 , whereas the tropical formula yields $\min \{6,6,7,8,7\}=6$.

(3) Finally, $\Delta\left(p_{3}\right)=4 t^{6}-8 t^{8}+4 t^{10}$, the valuation of which is 6 , and the tropical formula yields $\min \{8,6,10,8,8\}=6$.

Again, it is the valuations of the roots here leading to cancellation in the discriminant. We might ask precisely what conditions on the roots lead to this occuring - in this example, $p_{2}$ and $p_{3}$ both have two roots of valutaion 1 and one root of valuation 2 , yet the discriminant of $p_{2}$ has cancellation occuring whereas the discriminant of $p_{3}$ does not. The valuations of the coefficients for $p_{2}$ give a tropical root of the tropical formula for the discriminant, whereas the valuations of the coefficients of $p_{3}$ do not. This suggests that while the valuations of the roots can determine whether or not we will get cancellation in the discriminant, there are additional conditons that must also be met.

\section{Open Questions and Conclusion}

In Section 4.3 we saw that the discriminant of $f(x, z)=x^{2}+B x z+C z^{2}$ does appear as a factor in the Plücker coordinates of both $\langle y z+f(x, z)\rangle$ and $\left\langle y^{2}+f(x, z)\right\rangle$, and it seems reasonable to ask the following question and make the cautious prediction that the answer may be yes.

Question 6.0.1. Does the discriminant $\Delta_{3}=-4 A^{3}-27 B^{2}$ eventually appear as a factor in one of the Plücker coordinates of $\left\langle y^{2} z+x^{3}+A x z^{2}+\right.$ $\left.B z^{3}\right\rangle$ ?

This would mean that if two elliptic curves had different valuated $j$-invariants, their tropical linear spaces would also differ, and therefore looking at the tropical scheme structure might allow us to recover some data "lost" in the tropicalisation map, or at the very least tell us when data has been lost.

Now we will collect the various remaining unanswered questions posed in previous sections, which fall into two main categories: what we don't yet understand about the Plücker coordinates of some of the ideals studied, and questions relating to the validity of the results obtained over fields of characteristic $p \neq 0$. 
The first three questions fall into the first category, and answers or partial answers to these questions would allow us to further generalise the results obtained in previous sections.

Question 6.0.2. For a general cubic ideal in $\mathbb{P}^{1}$ of the form $\left\langle A x^{3}+\right.$ $\left.B x^{2} z+C x z^{2}+D z^{3}\right\rangle$, is there a neat way of describing all of the Plücker coordinates?

Question 6.0.3. Given a polynomial ideal $\langle f(x, z)\rangle$, homogeneous of degree $n$ in $\mathbb{P}^{1}$ with well-understood Plücker coordinates, is there a neat way of describing all of the Pluecker coordinates of the ideal $\left\langle y^{n}+\right.$ $f(x, z)\rangle$ ?

Question 6.0.4. Specifically for quadratics in $\mathbb{P}^{2}$, which submatrices of the Macaulay matrices give rise to Plücker coordinates that have the discriminant $\Delta$ as a factor?

The following three questions fall into the second category. It would be nice to understand how the results differ between fields of characteristics 0 and $p \neq 0$, and how results may differ for different values of $p$. The formula for the discriminant is not applicable when $p$ divides the degree of the polynomial, so it would be useful to have more information about these cases too.

Question 6.0.5. For an ideal I generated by a quadratic with coefficients in fields of characteristic 2 , using an appropriate analogue $\Delta^{\prime}$ to the usual discriminant, does the tropical ideal Trop $(I)$ determine the valuation of $\Delta^{\prime}$ ?

Question 6.0.6. Similarly, for ideals J generated by cubics (resp. elliptic curves) with coefficients in fields of characteristic 2 and 3, what can we say about the relationship between the tropical ideals Trop $(J)$ and the valuation of the (analogue to the) discriminant (resp. j-invariant)?

Question 6.0.7. For ideals $K$ generated by quadratics, or cubics of the form $x^{3}+A x z^{2}+B z^{3}$ with coefficients in fields of characteristic $p \neq 0$, for which values of $p$ does the tropical ideal Trop $(K)$ determine the valuation of the discriminant?

\section{REFERENCES}

[1] E. R. Berlekamp An Analog to the Discriminant over Fields of Characteristic Two, Journal of Algebra 38, 315-317, 1976

[2] Andrew J. Chan and Diane Maclagan Gröbner Bases Over Fields with Valuations arXiv:1303.0729v2

[3] David Cox, John Little and Hal Schenk Toric Varieties, AMS Graduate Studies in Mathematics, 2011

[4] David Eisenbud Commutative Algebra with a View Toward Algebraic Geometry

[5] David Eisenbud and Joe Harris The Geometry of Schemes, Springer, Graduate Texts in Mathematics 197, 2000 
[6] Bart Frenk Discriminants, Resultants and Multidimensional Determinants, Springer Science+Business Media New York, 1994

[7] Israel M. Gelford, Mikhail M. Kapranov, Andrei V. Zelevinsky Tropical Varieties, Maps and Gossip, http://alexandria.tue.nl/extra2/750815.pdf

[8] Jeffrey Giansiracusa and Noah Giansiracusa Equations of Tropical Varieties, 2016

[9] Alex Fink, Jeffry Giansiracusa and Noah Giansiracusa Projective Hypersurfaces in Tropical Scheme Theory, Unpublished

[10] Joe Harris Algebraic Geometry, Springer, Graduate Texts in Mathematics 133, 1992

[11] Robin Hartshorne Algebraic Geometry, Springer, Graduate Texts in Mathematics 52, 1977

[12] Eric Katz, Hannah Markwig, Thomas Markwig The j-invariant of a Plane Tropical Cubic, 2007

[13] Eric Katz, Hannah Markwig, Thomas Markwig The Tropical j-invariant, 2008

[14] Serge Lang Elliptic Functions, Springer-Verlag, Graduate Texts in Mathematics 112, 1987

[15] Diane MacLagan and Felipe Rincon Tropical Schemes, Tropical Cycles and Valuated Matroids, Journal of the European Mathematical Society, November 19, 2019

[16] Diane MacLagan and Felipe Rincon Tropical Ideals, Compositio Mathematica Volume 154, Issue 3, March 2018, pp. 640-670

[17] Diane MacLagan and Bernd Sturmfels Introduction to Tropical Geometry, AMS Graduate Studies in Mathematics 161, 2015

[18] James Oxley What is a Matroid?, 2014, https://www.math.lsu.edu/ oxley/survey4.pdf

[19] Lawrence C. Washington Elliptic Curves - Number Theory and Cryptography, CRC Press, Taylor \& Francis Group 2008 